\title{
MIMO Signal Processing in Offset-QAM Based Filter Bank Multicarrier Systems
}

\author{
Ana I. Pérez-Neira*, Senior Member, IEEE, Màrius Caus, Rostom Zakaria, Didier Le Ruyet, Senior \\ Member, IEEE, Eleftherios Kofidis, Member, IEEE, Martin Haardt, Senior Member, IEEE, Xavier Mestre, Senior \\ Member, IEEE and Yao Cheng, Student Member, IEEE
}

\begin{abstract}
Next generation communication systems have to comply with very strict requirements for increased flexibility in heterogeneous environments, high spectral efficiency and agility of carrier aggregation. This fact motivates research in advanced multicarrier modulation (MCM) schemes, such as filter bank-based multicarrier (FBMC) modulation. This paper focuses on the offset quadrature amplitude modulation (OQAM)based FBMC variant, known as FBMC/OQAM, which presents outstanding spectral efficiency and confinement in a number of channels and applications. Its special nature, however, generates a number of new signal processing challenges that are not present in other MCM schemes, notably, in orthogonal frequency division multiplexing (OFDM). In multiple-input multiple-output (MIMO) architectures, which are expected to play a primary role in future communication systems, these challenges are intensified, creating new interesting research problems and calling for new ideas and methods that are adapted to the particularities of the MIMO-FBMC/OQAM system. The goal of this paper is to focus on these signal processing problems and provide a concise yet comprehensive overview of the recent advances in this area. Open problems and associated directions for future research are also discussed.
\end{abstract}

\section{INTRODUCTION}

The ever increasing demands for digitalized anytime and anywhere contents have fueled an explosive growth of Internet access. With the proliferation of data-hungry applications, it is expected that in the next few years the number of devices with Internet connection will increase tremendously, leading to a massive wireless connectivity among users and machines. The unprecedented traffic increase in human- and machinetype communications poses stringent constraints in throughput, energy and delay. Strategies that are being considered to respond to these demands with the current spectrum scarcity

Copyright (c) 2015 IEEE. Personal use of this material is permitted. However, permission to use this material for any other purposes must be obtained from the IEEE by sending a request to pubs-permissions@ieee.org.

A.I. Pérez-Neira is with the Department of Signal Theory and Communications, Universitat Politècnica de Catalunya (UPC), 08034 Barcelona, Spain and with the Centre Tecnològic de Telecomunicacions de Catalunya (CTTC), 08860 Castelldefels, Barcelona, Spain (e-mail: ana.isabel.perez@upc.edu).

M. Caus and X. Mestre are with the Centre Tecnologic de Telecomunicacions de Catalunya (CTTC), 08860 Castelldefels, Barcelona, Spain (e-mail: \{marius.caus, xavier.mestre\}@cttc.cat

R. Zakaria and D. Le Ruyet are with CEDRIC/LAETITIA, CNAM, Paris, France (e-mail: \{didier.le ruyet, rostom.zakaria\}@cnam.fr).

E. Kofidis is with the Department of Statistics and Insurance Science, University of Piraeus, Piraeus 18534, Greece, and the Computer Technology Institute \& Press "Diophantus", Greece (CTI) (e-mail: kofidis@unipi.gr).

M. Haardt and Y. Cheng are with the Communications Research Laboratory, Ilmenau University of Technology, D-98684 Ilmenau, Germany (e-mail: \{martin.haardt, y.cheng\}@tu-ilmenau.de). include: cell densification, authorized spectrum sharing, millimeter wave (mm-wave) communications and large scale antenna arrays at the base station [1]. A popular view is that 5th generation $(5 \mathrm{G})$ systems will support the future needs through the combination of the above strategies. Hence, $5 \mathrm{G}$ is envisioned to provide spectrum flexibility and unleash the potential of multiple-input multiple-output (MIMO) technology. This anticipates that the waveform type and the MIMO solution to be adopted will play a decisive role in fulfilling the $5 \mathrm{G}$ requirements. An air interface that achieves a finegrained control of the spectrum and is well-suited to MIMO communication systems is bound to be adopted in upcoming $5 \mathrm{G}$ standard releases.

The dominant transmission technology nowadays is based on the orthogonal frequency division multiplexing (OFDM) scheme [2]. The beauty of OFDM stems from the ease of its implementation and its robustness against multipath fading, which allows to model the end-to-end communication system as a set of parallel frequency flat subchannels. These two reasons explain why OFDM has been the modulation of choice in most of the current wireless communication standards such as: Long-Term Evolution (LTE) [3], Worldwide Interoperability for Microwave Access (WiMAX) [4], wireless local area networks (WLAN) [5], digital video broadcasting-terrestrial (DVB-T) [6] and digital audio broadcasting (DAB) [7]. Next generation communication systems will require an orthogonal frequency division multiple access (OFDMA) scheme that presents better stop-band attenuation and allows for a flexible carrier aggregation, without the need of strict synchronization among users. In this context, enhanced versions of OFDM have been investigated, e.g., [8]-[12]. A viable alternative to enhanced OFDM is offered by filter bank-based multicarrier (FBMC) modulations [13], [14]. This is because FBMC offers the possibility of shaping subcarrier signals with waveforms that are well-localized in both frequency and time axes [15]. This translates into a steep sidelobe decay, allowing a flexible spectrum usage and offering an increased resilience against time and frequency misalignment, compared to enhanced versions of OFDM. With FBMC, carrier aggregation becomes a trivial task, since each subcarrier band is confined to an assigned range and has a negligible interference to other bands. As a consequence of its good properties, FBMC has played a central role in several recent and on-going international projects [16]-[20] and in the 5G infrastructure public-private partnership [21]. FBMC is being considered as the modulation of choice not only for 5G cellular networks [2], [18], [20], [22] 
but also for the professional mobile radio (PMR) evolution [17] and for satellite communications [23].

One of the most studied FBMC schemes is based on offset quadrature amplitude modulation (OQAM), usually referred to as OFDM/OQAM [24], [25]. It is also widely known as FBMC/OQAM or, less commonly, as staggered multitone (SMT) [14] and was in fact first proposed in the 60's [26]. Regarding the computational burden, the complexity analysis reveals that the necessary effort to implement the transceiver is higher in FBMC/OQAM than in OFDM [27]. However, realistic (semi-realtime) demonstrations have shown a significant spectral efficiency gain (of the order of 20-30\%) for FBMCbased systems [16], justifying the extra computational cost.

In view of the successful combination of OFDM with MIMO, the objective of this paper is to show that FBMC/OQAM can also benefit from the advantages and gains of incorporating MIMO processing. The latest signal processing developments that enable the combination of MIMO and FBMC/OQAM are the subject of this overview paper. Many other multicarrier modulation schemes are currently under investigation: universal filtered-multicarrier (UFMC) [28], [29], generalized frequency division multiplexing (GFDM) [30][33], filtered multitone (FMT) [34], [35] and cyclic block filtered multitone (CB-FMT) modulation [36]. UFMC, GFDM and CB-FMT also possess the key ingredients to become the new wide band access scheme. The criterion that tips the balance towards the winner has not been established yet, but aspects such as spectral efficiency, power spectral density, complexity, sensitivity to multipath fading and applicability in MIMO architectures are crucial to identify the most competitive modulation. This paper focuses on the FBMC/OQAM scheme because it is the most spectrally efficient alternative, attaining the highest symbol density in the time-frequency plane [15]. Moreover, it exhibits a good spectral confinement [37], [38] and does not rely on the CP transmission [25]. The latter may however complicate the channel equalization task and hence there have been a number of alternative proposals that rely on the insertion of a CP [37]-[40]. This paper does not consider enhancements related to the features of the final implementation. This work gives priority to the original FBMC/OQAM waveform, which presents outstanding spectral efficiency, and studies the challenges that it poses to MIMO processing. Therefore, CP-based variants of FBMC/OQAM will not be covered.

Differently to OFDM, the use of FBMC/OQAM in multiantenna configurations is not as straightforward. In general terms, one cannot rely on a mere generalization of the solutions adopted in OFDM-based systems (as it is often the case for other filtered multicarrier schemes aided with a $\mathrm{CP})$. Early works in the literature that compared OFDM and FBMC/OQAM for multistream MIMO systems did not take into account the different signal structure of FBMC/OQAM and how to take advantage of it [41]. Indeed, how to fully exploit the potentials of FBMC/OQAM in MIMO systems is still an open research problem. This paper overviews previous works and also establishes the foundations to designing the basic techniques, such as channel inversion and channel diagonalization.
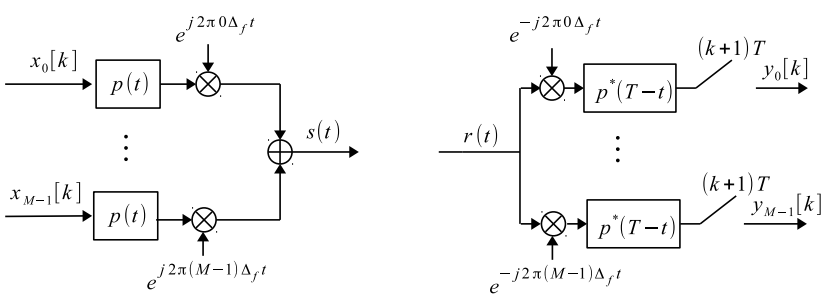

Figure 1. Block diagram of a parallel data transmission system.

The rest of the paper is organized as follows. In the next section, the FBMC/OQAM system model is described and the two cases that correspond to different degrees of channel frequency selectivity are outlined. In Section III, we address the design of MIMO techniques for FBMC/OQAM systems that achieve multiplexing and diversity gains by exclusively using the knowledge of the channel state information (CSI) at the receive side. The techniques described in Section IV benefit from the CSI knowledge at both ends of the link to jointly design the transmitter and the receiver. The possibility of using CSI at transmission (CSIT) opens the door to allocating several users over the same frequency resources. As a consequence, Section IV encompasses single- and multiuser communication systems. All of the above mentioned techniques require estimates of the MIMO channels. Channel estimation techniques for MIMO-FBMC/OQAM are reviewed in Section V. Finally, Section VI concludes the paper and discusses directions for future research.

Notation: Upper- and lower-case boldface letters denote matrices and vectors, respectively. Let the superscripts $(.)^{T},(.)^{*}$ and $(.)^{H}$ denote, respectively, transpose, complex conjugate and Hermitian transpose operations. We will use $[\mathbf{A}]_{i j}$ to refer to the $(i, j)$ th element of a matrix $\mathbf{A}$. By $\mathbf{I}_{N}$ we denote the $N$-th order identity matrix. We define $\lambda_{l}(\mathbf{A})$ to be the $l$ th largest eigenvalue of a matrix A. $\operatorname{diag}\left\{a_{1}, \ldots, a_{N}\right\}$ is an $N \times N$ diagonal matrix, the $(k, k)$ th element of which is given by $a_{k}$. We will use $\star$ to denote the convolution operation. Let $\operatorname{tr}($.$) and$ $\mathbb{E}\{\cdot\}$ be the trace and expectation operators, respectively. $\otimes$ is the (left) Kronecker product. The Frobenius norm of a matrix $\mathbf{A}$ is denoted by $\|\mathbf{A}\|_{F}$. The symbol $\delta_{k, n}$ is 1 if $k=n$ and 0 otherwise. $\sqrt{-1}$ is denoted by $j$. The sets of $N \times M$ real- and complex-valued matrices are respectively denoted by $\mathbb{R}^{N \times M}$ and $\mathbb{C}^{N \times M} . \Re(\mathbf{A})$ and $\Im(\mathbf{A})$ stand for the real and imaginary parts of $\mathbf{A} \in \mathbb{C}^{N \times M}$, respectively. The extended version of a matrix $\mathbf{A}$ is defined as $\overline{\mathbf{A}}=\left[\Re\left(\mathbf{A}^{T}\right) \Im\left(\mathbf{A}^{T}\right)\right]^{T}$. The upsampling and downsampling operations by a factor of $x$ will be denoted by $(.)_{\uparrow x}$ and $(.)_{\downarrow x}$, respectively.

\section{SySTEM MODEL}

The aim of this section is to present a formulation for the FBMC/OQAM modulation scheme that unifies single-inputsingle-output (SISO) and MIMO architectures. The SISO FBMC/OQAM transmission format hinges on introducing a time offset on the real or imaginary (depending on the parity of the subcarrier) part of the QAM symbols, which is equal to half the symbol period. This is tantamount to the transmission of pulse amplitude-modulated (PAM) data symbols at the 

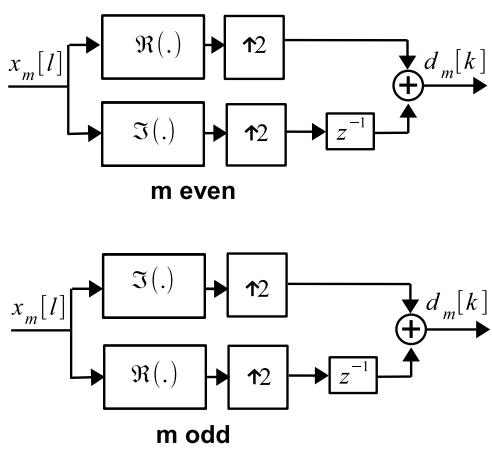

Figure 2. OQAM staggering in a multicarrier structure.

double rate with a phase shift of $\frac{\pi}{2}$ inserted between adjacent symbols along time and frequency axes. The result is a weak (real-domain) subcarrier orthogonality, characterized by the presence of an intrinsic self-interference at the received signal even when no distortion is present in the transmission link. This self-interference can be easily removed under ideal transmission conditions since it is in quadrature with the desired signal. In the presence of multipath fading however, additional signal processing is required to restore the perfect reconstruction (PR) property. Next subsection describes the SISO transmission format in detail.

\section{A. SISO}

This section provides the basics of a parallel QAM transmission system [42], which will pave the way to understanding the idea behind FBMC/OQAM. In this work, the most common case of a uniformly modulated filter bank is considered. This system is depicted in Fig. 1. The filters of the filter bank are time-frequency shifted versions of the prototype pulse $p(t)$. The parallel data transmission concept consists in splitting the bandwidth into $M$ subbands, which are spaced $\Delta_{f}$ apart in frequency. At each subband, symbols are pulse shaped by $p(t)$ and transmitted with rate $\frac{1}{T}$, where $T$ stands for the symbol period. The matched filter is represented by $p^{*}(T-t)$, which is time-shifted so as to be causal, and $(k+1) T$ corresponds to the sampling instant. This strategy allows the system to achieve a fine-grained control of the spectrum, because $p(t)$ is a bandlimited low-pass filter. A common practice for simplifying the waveform design and increasing the bandwidth efficiency is to use a spectral roll-off higher than zero and allow spectral overlapping.

According to Fig. 1, data can be recovered under ideal channel conditions only if

$$
\begin{aligned}
& \int p(t-k T) e^{j 2 \pi m \Delta_{f} t} \times \\
& p^{*}\left(t-k^{\prime} T\right) e^{-j 2 \pi m^{\prime} \Delta_{f} t} d t=\delta_{k^{\prime}, k} \delta_{m^{\prime}, m}
\end{aligned}
$$

This orthogonality condition imposes a constraint, $\frac{1}{\Delta_{f} T} \leq 1$, on the symbol density [24]. A prototype pulse that satisfies this constraint with equality, corresponding to minimum symbol spacing, cannot be well-localized both in time and frequency [24]. A well known example is given by the rectangular pulse, which underlies classical OFDM. It turns out, however, that
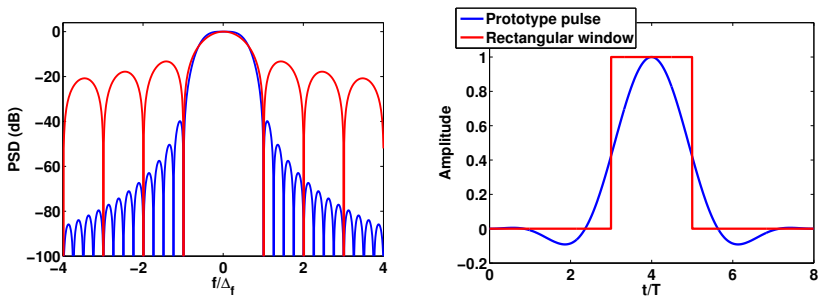

Figure 3. Power spectral density and time response of the prototype pulse [44] and the rectangular window. The frequency is normalized to the subcarrier spacing and the time is normalized to the symbol period.

good time-frequency localization (TFL) with $\frac{1}{\Delta_{f} T}=1$ is possible if OQAM modulation is employed, by alternately shifting the parallel data transmissions in time $(T / 2)$ and in frequency $(\pi / 2)$. A detailed explanation can be found in [43]. The baseband transmitted signal can then be written as

$$
\begin{aligned}
s(t)= & \sum_{k \in \mathbb{Z}} \sum_{m \text { even }}\left(\Re\left(x_{m}[k]\right) p(t-k T)+\right. \\
& \left.j \Im\left(x_{m}[k]\right) p(t-T / 2-k T)\right) e^{j 2 \pi m \Delta_{f} t}+ \\
& \sum_{k \in \mathbb{Z}} \sum_{m \text { odd }}\left(j \Im\left(x_{m}[k]\right) p(t-k T)+\right. \\
& \left.\Re\left(x_{m}[k]\right) p(t-T / 2-k T)\right) e^{j 2 \pi m \Delta_{f} t},
\end{aligned}
$$

where $x_{m}[k]$ is the QAM complex-valued symbol transmitted on the $m$ th subcarrier and at the $k$ th time instant. Notice that the symbols on adjacent subcarriers are shifted by a phase of $\frac{\pi}{2}$. To simplify the notation, it is useful to realize that OQAM introduces a staggered structure, which is equivalent to transmitting PAM symbols with rate $\frac{2}{T}$. By defining

$$
\begin{gathered}
d_{2 m}[2 k]=\Re\left(x_{2 m}[k]\right), \quad d_{2 m+1}[2 k]=\Im\left(x_{2 m+1}[k]\right) \\
d_{2 m}[2 k+1]=\Im\left(x_{2 m}[k]\right), \quad d_{2 m+1}[2 k+1]=\Re\left(x_{2 m+1}[k]\right) \\
\phi_{m}[k]=e^{j\left(\frac{\pi}{2}(m+k)-\pi m k\right)}
\end{gathered}
$$

the FBMC/OQAM signal $s(t)$ can alternatively be written as

$$
s(t)=\sum_{k \in \mathbb{Z}} \sum_{m=0}^{M-1} d_{m}[k] \phi_{m}[k] p\left(t-k \frac{T}{2}\right) e^{j 2 \pi m \Delta_{f} t} .
$$

Fig. 2 illustrates how the OQAM sequences are generated by staggering between the in-phase and quadrature components of the QAM symbol $x_{m}[l]$. The orthogonality condition, which guarantees that symbols $\left\{d_{m}[k]\right\}$ are perfectly recovered at the receive side, is then expressed as

$$
\begin{aligned}
& \Re\left(\int p\left(t-k \frac{T}{2}\right) e^{j 2 \pi m \Delta_{f} t} \phi_{m}[k] \times\right. \\
& \left.p^{*}\left(t-k^{\prime} \frac{T}{2}\right) e^{-j 2 \pi m^{\prime} \Delta_{f} t} \phi_{m^{\prime}}^{*}\left[k^{\prime}\right] d t\right)=\delta_{k^{\prime}, k} \delta_{m^{\prime}, m},
\end{aligned}
$$

in contrast to (1). 


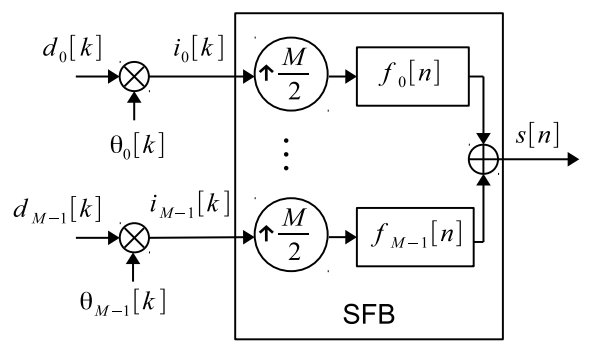

Figure 4. Block diagram of the FBMC/OQAM transceiver.

1) From continous-time to discrete-time FBMC/OQAM: Defining the critical sampling period by $T_{s}=\frac{T}{M}$, the prototype pulse can be obtained by sampling an analog waveform as follows: $p[n]=p\left((n-D) T_{s}\right)$. The delay $D T_{s}$ is set to guarantee that $p[n]$ is causal. When $p(t)$ is truncated to this interval $\left[-(L / 2) T_{s},(L / 2) T_{s}\right]$, as proposed in [25], then it follows that $D=(L-1) / 2$ and $p[n]$ is defined for $n=0,1, \ldots, L-1$. The prototype pulse $p[n]$ is usually designed to be evenly symmetric and confined within the frequency range $\left[-\frac{2 \pi}{M}, \frac{2 \pi}{M}\right]$. The length of the pulse is usually expressed as $L=K M$, where $K$ is the overlapping factor. As a result, the higher the overlapping factor is, the faster the pulse can decay in the frequency domain. There are several pulses that achieve a good frequency confinement with $K \leq 4$, see, e.g., [24], [25], [44]-[48]. Note that some of them are directly designed in the discrete-time domain, because the pulses that are obtained by truncation and discretization of $p(t)$ lose the orthogonality. Fig. 3 shows the good spectral characteristics exhibited by the design proposed in [44] with $K=4$ and $M=1024$.

The discrete-time baseband model for FBMC/OQAM is expressed as

$$
s[n]=\sum_{k \in \mathbb{Z}} \sum_{m=0}^{M-1} d_{m}[k] \theta_{m}[k] f_{m}\left[n-k \frac{M}{2}\right],
$$

where

$$
\begin{gathered}
\theta_{m}[k]=e^{j \frac{\pi}{2}(m+k)} \\
f_{m}[n]=p[n] e^{j \frac{2 \pi}{M} m(n-D)} .
\end{gathered}
$$

Note that low-rate signals, i.e. the PAM modulated symbols, use the sampling index $k$ while the high-rate signals are indexed by $n$. The signal formulated in (7) reveals that the tails of $p[n]$ result in an overhead that cannot be neglected in short burst transmission. This disadvantage is avoided by truncating the initial and final transients of the burst [49], yet the distortion and the out-of-band radiation is then substantially increased. These drawbacks can be overcome to some extent by transmitting virtual symbols and subsequently applying a window function in the time domain [50]. Alternatively, the circular convolution can be adopted followed by a tail biting operation [51]. However, since the circular structure brings about sharp edge transitions, it is imperative to use a time window to reduce the increased sidelobes. In the rest of the paper, none of these alternatives will be considered and we will stick to the notation used in (7) for ease of exposition.

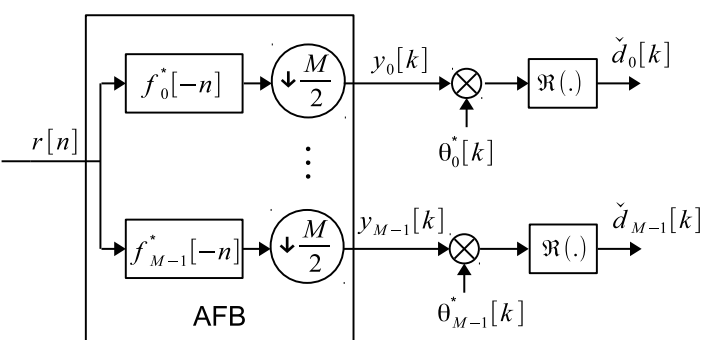

In this case, it is worth emphasizing that the set of functions $\left\{\theta_{m}[k] f_{m}\left[n-\frac{M}{2} k\right]\right\}$ constitutes an orthonormal basis in the real domain if

$$
\Re\left(\theta_{q}^{*}[k] \theta_{m}[k-\tau] \alpha_{q m}[\tau]\right)=\Re\left(\Gamma_{q m}^{k}[\tau]\right)=\delta_{q, m} \delta_{\tau, 0},
$$

where the transmultiplexer response

$$
\Gamma_{q m}^{k}[\tau]=\theta_{q}^{*}[k] \theta_{m}[k-\tau] \alpha_{q m}[\tau]
$$

depends on the inner product between $f_{m}\left[n+\tau \frac{M}{2}\right]$ and $f_{q}^{*}[n]$ given by

$$
\alpha_{q m}[\tau]=\sum_{n} f_{m}\left[n+\tau \frac{M}{2}\right] f_{q}^{*}[n] .
$$

The variable $\alpha_{q m}[\tau]$ can be understood as the coupling between two symbols that are separated $(q-m) \frac{1}{T}$ in frequency and $\tau \frac{T}{2}$ in time. The condition (10) is unchanged if the phase term is defined as

$$
\theta_{m}[k]=\left\{\begin{array}{lll}
1, & k+m & \text { even } \\
j, & k+m & \text { odd },
\end{array}\right.
$$

instead of (8). In practice, both definitions are used. Based on the discrete-time formulation, the FBMC/OQAM transceiver can be depicted as Fig. 4 shows. It is worth mentioning that the synthesis filter bank (SFB) and the analysis filter bank (AFB) can be efficiently implemented using the inverse fast Fourier transform (IFFT) and the FFT, respectively [25]. The block diagram shows that there are $M$ input data symbols that are upsampled, filtered and then, added to form the composite signal $s[n]$. It is important to remark that upsampling controls the symbol period and compresses the signal spectrum by a factor $\frac{M}{2}$, by introducing $\frac{M}{2}-1$ zeros between input symbols. It can be inferred from Fig. 4 that real-valued data symbols are transmitted at rate $f_{s} \frac{2}{M}$, where $f_{s}=\frac{1}{T_{s}}$ is the sampling frequency. The symbol that is fed into the $m$ th input is given by the real PAM data symbol $d_{m}[k]$ multiplied by the phase factor $\theta_{m}[k]$.

Under realistic propagation conditions, the signal $s[n]$ is affected by multipath fading and additive noise, which are denoted by $h[n]$ and $w[n]$, respectively. The demodulated signal on the $q$ th subcarrier, which is given by $y_{q}[k]=$ $\left(f_{q}^{*}[-n] \star(s[n] \star h[n]+w[n])\right)_{\downarrow \frac{M}{2}}$ is compactly written as

$$
y_{q}[k]=\sum_{m=q-1}^{q+1}\left(d_{m}[k] \theta_{m}[k]\right) \star g_{q m}[k]+w_{q}[k] .
$$


Table I

INTRINSIC INTERFERENCES UNDER IDEAL PROPAGATION CONDITIONS FOR $q$ EVEN AND THE PULSE OF [44]

\begin{tabular}{|c|c|c|c|c|c|c|c|c|c|c|c|c|}
\hline & $k=-5$ & $k=-4$ & $k=-3$ & $k=-2$ & $k=-1$ & $k=0$ & $k=1$ & $k=2$ & $k=3$ & $k=4$ & $k=5$ & $k=0.258$ \\
\hline$\alpha_{q q-1}[k]$ & $j 0.0013$ & 0.0054 & $-j 0.0429$ & -0.1250 & $j 0.2058$ & 0.2393 & $-j 0.2058$ & -0.1250 & $j 0.0429$ & 0.0054 & $-j 0.0013$ \\
\hline$\alpha_{q q}[k]$ & 0.0023 & 0 & -0.0668 & 0 & 0.5644 & 1 & 0.5644 & 0 & -0.0668 & 0 & 0.0023 \\
\hline$\alpha_{q q+1}[k]$ & $-j 0.0013$ & 0.0054 & $j 0.0429$ & -0.1250 & $-j 0.2058$ & 0.2393 & $j 0.2058$ & -0.1250 & $-j 0.0429$ & 0.0054 & $j 0.0013$ \\
\hline
\end{tabular}

The sum is typically restricted to the subcarriers $\{q-1, q, q+1\}$ only, because the subcarrier spacing is $\frac{2 \pi}{M}$ and the energy of the prototype pulse is confined within the interval $\left[-\frac{2 \pi}{M}, \frac{2 \pi}{M}\right]$, although the frequency response of $p[n]$ stretches over $[-\pi, \pi]$. According to the above model, interference can be classified as inter-symbol interference (ISI) or inter-carrier interference (ICI) as follows:

$$
\begin{aligned}
y_{q}[k]= & g_{q q}[0] d_{q}[k] \theta_{q}[k]+\underbrace{\sum_{\tau \neq 0} g_{q q}[\tau] d_{q}[k-\tau] \theta_{q}[k-\tau]}_{\text {ISI }} \\
& +\underbrace{\sum_{m \neq q} \sum_{\tau} g_{q m}[\tau] d_{m}[k-\tau] \theta_{m}[k-\tau]}_{\text {ICI }}+w_{q}[k] .
\end{aligned}
$$

If $q=0$, the interfering signals come from subcarriers $\{0,1, M-1\}$, due to the periodicity of the discrete-time Fourier transform (DTFT). Analogously, the signals that contaminate the $M$ th output of the AFB, i.e. $y_{M-1}[k]$, leak from subcarriers $\{0, M-2, M-1\}$. In (15), the magnitudes of $\left\{g_{q m}[k]\right\}$ are such that the desired and the unwanted signals can be of comparable strengths, which shows the importance and difficulty of the channel equalization. It will be shown in the subsequent sections that the equalization task is greatly simplified if the channel frequency response is sufficiently smooth in the pass band region of one subcarrier. In this case, all the interfering signals that come from a given subcarrier experience the same channel, up to a constant factor. However, this simplification does not hold true if the subcarrier spacing is significantly higher than the channel coherence bandwidth. Therefore, it can be stated that the degree of the channel frequency selectivity affects the channel expression, so that the equivalent response from subcarrier $m$ to subcarrier $q$ can be formulated using these two models

$$
\begin{aligned}
& \text { 1) } g_{q m}[k]=\left(f_{m}[n] \star h[n] \star f_{q}^{*}[-n]\right)_{\downarrow \frac{M}{2}} \\
& \text { 2) } g_{q m}[k] \approx H\left(\frac{2 \pi}{M} m\right) \alpha_{q m}[k],
\end{aligned}
$$

where $H(\omega)=\sum_{n} h[n] e^{-j \omega n}$ is the DTFT of $h[n]$ evaluated at the radial frequency $\omega$. With even $q$, and with the prototype pulse being designed as described in [44] with an overlapping factor of $K=4, \alpha_{q m}[k]$ takes the values shown in Table I. When $q$ is odd the same table is valid albeit with some sign changes. To better appreciate the approximation error in (14), the power that leaks from $q-2$ and $q+2$ has been computed. In view of the fact that $\sum_{k}\left(\left|\alpha_{q q-2}[k]\right|^{2}+\left|\alpha_{q q+2}[k]\right|^{2}\right)=$ $2.85 \times 10^{-6}$ and $\left|\alpha_{q q}[0]\right|^{2}=1$, we can conclude that the mismatch modeling error is negligible.

Regarding the distinction made in (16), model 1) accounts for the most general case and it is always valid. The simplified

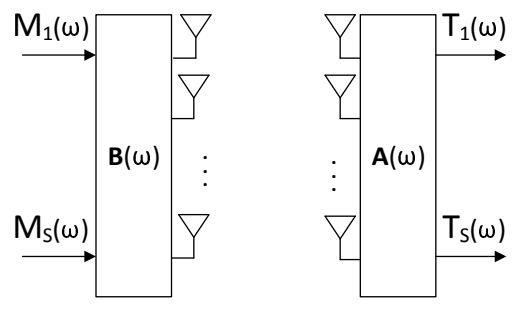

Figure 5. Ideal implementation of a frequency precoder $\mathbf{B}(\omega)$ and a frequency equalizer $\mathbf{A}(\omega)$.

model 2) hinges on assuming that the channel frequency response is flat at the subcarrier level. Note that in model 2) the channel seen by the signal that comes from the $m$ th subcarrier is flat and equal to $H\left(\frac{2 \pi}{M} m\right)$. It must be mentioned that model 2) is preferable over model 1) because it offers a better analytical tractability. However, system parameters and propagation conditions will determine its validity. From this point on, we will be using the term low frequency selective channels to refer to those scenarios where model 2) is valid. Otherwise, the term highly frequency selective channels will be used.

\section{B. $M I M O$}

In the SISO case, $M$ streams are transmitted in parallel, i.e. one per frequency bin. Let us now consider the case where the transmitter and the receiver are equipped with $N_{T}$ and $N_{R}$ antennas, respectively. Using the spatial degrees of freedom, $S$ multicarrier signals can be multiplexed. Fig. 5 depicts the most general architecture to implement any linear multicarrier MIMO transceiver. If the DTFT of $d_{m}^{l}[k]$ is defined as $D_{m}^{l}(\omega)$, the DTFT of the symbols associated with the $l$ th substream and the $m$ th subcarrier becomes

$$
I_{m}^{l}(\omega)=\sum_{k} d_{m}^{l}[k] \theta_{m}[k] e^{-j \omega k}=j^{m} D_{m}^{l}\left(\omega-\frac{\pi}{2}\right),
$$

for $l=1,2 \ldots, S$. Then, the DTFT of the transmitted signal associated with the $l$ th substream can be expressed as

$$
M_{l}(\omega)=\sum_{m=0}^{M-1} F_{m}(\omega) I_{m}^{l}\left(\frac{\omega M}{2}\right) .
$$

It should be mentioned that the spectrum of the symbols is shrunk by a factor $M / 2$ after performing the upsampling operation, leading to $I_{m}^{l}\left(\frac{\omega M}{2}\right)$. According to the definition given in (9), $F_{m}(\omega)$ can be written as

$$
F_{m}(\omega)=e^{-j \frac{2 \pi}{M} m D} P\left(\omega-\frac{2 \pi m}{M}\right),
$$



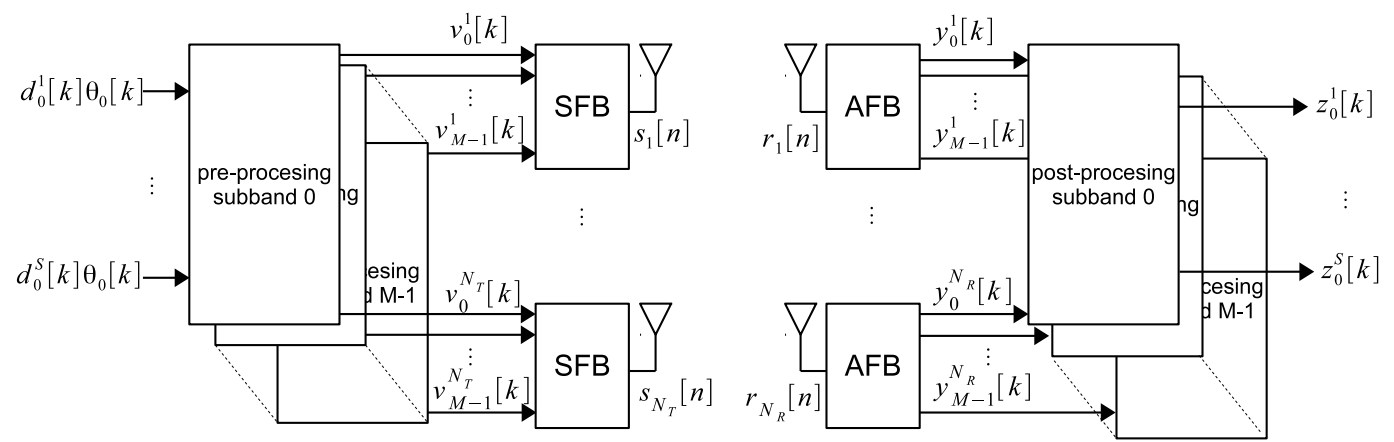

Figure 6. Transmitter and receiver structure of multi-stream MIMO-FBMC/OQAM systems with precoders and equalizers working on a per-subchannel basis.

where $P(\omega)$ is the DTFT of the prototype pulse $p[n]$. The frequency representation of the $S$ parallel FBMC/OQAM modulated streams is denoted by

$$
\mathbf{M}(\omega)=\left[M_{1}(\omega) \cdots M_{S}(\omega)\right]^{T}=\sum_{m=0}^{M-1} F_{m}(\omega) \mathbf{I}_{m}\left(\frac{\omega M}{2}\right)
$$

The lth entry of $\mathbf{I}_{m}(\omega) \in \mathbb{C}^{S \times 1}$ is equal to $I_{m}^{l}(\omega)$. Denoting by $\mathbf{B}(\omega) \in \mathbb{C}^{N_{T} \times S}$ the linear precoder, by $\mathbf{H}(\omega) \in \mathbb{C}^{N_{R} \times N_{T}}$ the MIMO channel and by $\mathbf{A}(\omega) \in \mathbb{C}^{N_{R} \times S}$ the equalizer, the received signal before the AFB takes the form

$$
\mathbf{T}(\omega)=\mathbf{A}^{H}(\omega) \mathbf{H}(\omega) \mathbf{B}(\omega) \mathbf{M}(\omega)+\mathbf{A}^{H}(\omega) \mathbf{W}(\omega) .
$$

Notice that the frequency representation of the equalized signal is given by $\mathbf{T}(\omega)=\left[T_{1}(\omega) \cdots T_{S}(\omega)\right]^{T}$ and the additive noise is denoted by $\mathbf{W}(\omega)=\left[W_{1}(\omega) \cdots W_{N_{R}}(\omega)\right]^{T}$. The components of these vectors are defined as follows: $T_{i}(\omega)=$ $\sum_{n} t_{i}[n] e^{-j \omega n}$ and $W_{j}(\omega)=\sum_{n} w_{j}[n] e^{-j \omega n}$, for $1 \leq i \leq S$ and $1 \leq j \leq N_{R}$. In these expressions, $t_{i}[n]$ denotes the sequence at the input of the $i$ th AFB and $w_{j}[n]$ is the additive noise that contaminates the reception of the $j$ th antenna.

The implementation of the architecture depicted in Fig. 5 may be extremely difficult if the impulse responses of the precoder and the equalizer are excessively long. This is the case with the singular value decomposition (SVD) beamforming, which relies on the SVD of polynomial matrices in time-dispersive media [52]. This problem is circumvented to some extent in multicarrier modulations, in particular for FBMC/OQAM systems, through decomposing the broadband MIMO channel into a set of subchannels, so that transmit and receive processing can be performed on a per-subchannel basis. Since the frequency selectivity of the subchannels is milder than that of the whole channel, the number of taps required to apply the pre- and the post-processing per subchannel is substantially reduced. In the least complex case, a single tap suffices. Fig. 6 shows the placement of precoding and equalization stages in an FBMC/OQAM transceiver. At the transmit side, $S$ streams are spatially multiplexed on each subcarrier. By means of the pre-processing stage, the vector $\theta_{m}[k] \mathbf{d}_{m}[k]=\mathbf{i}_{m}[k]=\theta_{m}[k]\left[d_{m}^{1}[k] \cdots d_{m}^{S}[k]\right]^{T}$ is mapped onto $N_{T}$ antennas, resulting in the column vector $\mathbf{v}_{m}[k]=\left[v_{m}^{1}[k] \cdots v_{m}^{N_{T}}[k]\right]^{T}$, for $0 \leq m \leq M-1$. Different alternatives to design the mapping will be analyzed in Section IV.

From (14), it can be inferred that the demodulated data at the $j$ th receive antenna on the $q$ th subcarrier is given by

$$
\begin{gathered}
y_{q}^{j}[k]=\sum_{m=q-1}^{q+1} \sum_{i=1}^{N_{T}} v_{m}^{i}[k] \star g_{q m}^{j i}[k]+w_{q}^{j}[k] \\
g_{q m}^{j i}[k]=\left(f_{m}[n] \star h_{j i}[n] \star f_{q}^{*}[-n]\right)_{\downarrow \frac{M}{2}} \\
w_{q}^{j}[k]=\left(w_{j}[n] \star f_{q}^{*}[-n]\right)_{\downarrow \frac{M}{2}},
\end{gathered}
$$

where the impulse response $h_{j i}[n]$ accounts for the channel between the $i$ th transmit antenna and the $j$ th receive antenna. The equivalent channel $g_{q m}^{j i}[k]$ is different from zero for $-L_{1}=-\left\lfloor\frac{L-1}{M / 2}\right\rfloor \leq k \leq\left\lfloor\frac{L-1+L_{\mathrm{ch}}}{M / 2}\right\rfloor=L_{2}$, where $L_{\mathrm{ch}}$ is the maximum of all channel lengths. Concerning the statistical information, it will be assumed that the symbols satisfy $\mathbb{E}\left\{d_{q}^{i}[k] d_{m}^{j}[l]\right\}=E_{S} \delta_{i, j} \delta_{q, m} \delta_{k, l}$ and that the noise samples are zero-mean circularly symmetric complex Gaussian random variables, i.e. $w_{j}[n] \sim \mathcal{C N}\left(0, N_{0}\right)$.

To counteract the channel and successfully detect the symbols, the vector $\mathbf{y}_{q}[k]=\left[y_{q}^{1}[k] \cdots y_{q}^{N_{R}}[k]\right]^{T}$ associated with the $q$ th subcarrier is post-processed to obtain $\mathbf{z}_{q}[k]=$ $\left[z_{q}^{1}[k] \cdots z_{q}^{S}[k]\right]^{T}$. This notation is sufficiently general to accommodate all the equalization techniques addressed in Sections III and IV. The signal at the output of the AFB can be compactly written using the following notation

$$
\begin{aligned}
\mathbf{y}_{q}[k] & =\sum_{m=q-1}^{q+1} \mathbf{G}_{q m}[k] \star \mathbf{v}_{m}[k]+\mathbf{w}_{q}[k] \\
& =\sum_{m=q-1}^{q+1} \sum_{\tau=-L_{1}}^{L_{2}} \mathbf{G}_{q m}[\tau] \mathbf{v}_{m}[k-\tau]+\mathbf{w}_{q}[k] \\
& =\mathbf{G}_{q q}[0] \mathbf{v}_{q}[k]+\sum_{(m, \tau) \in \Omega_{q, k}} \mathbf{G}_{q m}[\tau] \mathbf{v}_{m}[k-\tau]+\mathbf{w}_{q}[k],
\end{aligned}
$$

where $\mathbf{w}_{q}[k]=\left[w_{q}^{1}[k] \cdots w_{q}^{N_{R}}[k]\right]^{T}$. Note that the convolution between two sequences of matrices is defined as $\mathbf{A}[k] \star \mathbf{B}[k]=\sum_{l} \mathbf{A}[l] \mathbf{B}[k-l]$. In contrast to (14), the MIMO system formulated in (25) introduces interference between 


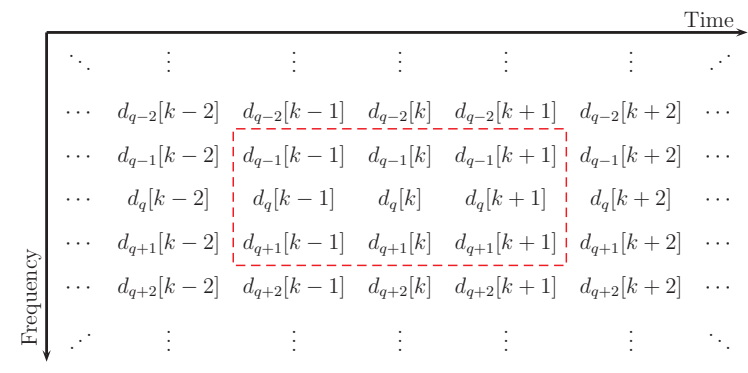

Figure 7. The neighborhood of the frequency-time point $(q, k)$. The dashed line box delimits the first-order neighborhood.

streams and antennas. Notice that, in view of the good TFL of the prototype filter, the intrinsic interference mainly comes from a neighborhood around the frequency-time (FT) position $(q, k)$, as Fig. 7 illustrates. The set $\Omega_{q, k}$ contains the FT positions that contribute to the interference. The equivalent channel matrix for each of the two models defined previously in (16) is given by

$$
\begin{aligned}
\text { 1) } \mathbf{G}_{q m}[\tau] & =\left[\begin{array}{ccc}
g_{q m}^{11}[\tau] & \cdots & g_{q m}^{1 N_{T}}[\tau] \\
\vdots & \ddots & \vdots \\
g_{q m}^{N_{R} 1}[\tau] & \cdots & g_{q m}^{N_{R} N_{T}[\tau]}
\end{array}\right] \\
\text { 2) } \mathbf{G}_{q m}[\tau] \approx \alpha_{q m}[\tau] \mathbf{H}_{m} . &
\end{aligned}
$$

To model case 2), which corresponds to a multiplicative channel distortion, the frequency response of the MIMO channel evaluated at $\frac{2 \pi}{M} m$ has been considered, namely

$$
\begin{aligned}
\mathbf{H}_{m} & =\left[\begin{array}{ccc}
H_{11}\left(\frac{2 \pi}{M} m\right) & \cdots & H_{1 N_{T}}\left(\frac{2 \pi}{M} m\right) \\
\vdots & \ddots & \vdots \\
H_{N_{R} 1}\left(\frac{2 \pi}{M} m\right) & \cdots & H_{N_{R} N_{T}}\left(\frac{2 \pi}{M} m\right)
\end{array}\right] \\
& =\mathbf{H}\left(\frac{2 \pi}{M} m\right) .
\end{aligned}
$$

It deserves to be highlighted that the frequency-domain formulation of the equivalent channel between the $m$ th and the $q$ th subcarrier reads

$$
\mathbf{G}_{q m}(\omega)=\left[F_{q}^{*}(\omega) F_{m}(\omega) \mathbf{H}(\omega)\right]_{\downarrow M / 2} .
$$

For any matrix $\mathbf{M}(\omega)$, the decimation by a factor $M / 2$ is represented by

$$
[\mathbf{M}(\omega)]_{\downarrow M / 2}=\frac{2}{M} \sum_{l=0}^{M / 2-1} \mathbf{M}\left(\frac{\omega-2 \pi l}{M / 2}\right) .
$$

Observe that when the per-subcarrier flat fading condition is satisfied, the frequency response becomes

$$
\mathbf{G}_{q m}(\omega) \approx\left[F_{q}^{*}(\omega) F_{m}(\omega)\right]_{\downarrow M / 2} \mathbf{H}_{m} .
$$

\section{Application of FBMC/OQAM in massive MIMO}

Recently, different variants of FBMC/OQAM have been studied in the context of massive MIMO communications [54], $[55]^{1}$. The essence of massive MIMO is in mitigating the

\footnotetext{
${ }^{1}$ The original study in [55] focused on the massive MIMO performance of cosine modulated multitone (CMT) modulations. However, it is pointed out in the same paper that similar conclusions hold for SMT (FBMC/OQAM).
}

effects of multi-user interference through the exploitation of a large number of antennas at the base station. The combination of massive MIMO and FBMC/OQAM is of the utmost importance to get the best of both technologies. Interestingly, the authors in [55] have experimentally verified that as the number of antennas increases, the equivalent channel after combining the signal components from different antennas becomes smooth, so that it can be assumed flat at the subcarrier level. This flattening effect, known as self-equalization, allows us to establish as accurate model 2) in (26), even for a subcarrier spacing of $87.5 \mathrm{kHz}$ over the Stanford University Interim 4 (SUI4) channel model [54]. Hence, multi-user MIMO techniques relying on model 2) will be valid for FBMC/OQAM-based massive MIMO communications, subject to self-equalization.

\section{Widely linear processing}

Given the channel output written in (25), the most common approach to estimating the data $\mathbf{d}_{q}[k]$ relies on exploiting the second-order statistics of $\mathbf{y}_{q}[k]$. Data symbols are drawn from a PAM constellation and, therefore, the vector $\mathbf{d}_{q}[k]$ exhibits non-circularity, which means that its pseudo-autocorrelation does not vanish, i.e. $\mathbb{E}\left\{\mathbf{d}_{q}[k] \mathbf{d}_{q}^{T}[k]\right\} \neq \mathbf{0}$ [56]. Hence, the autocorrelation $\mathbb{E}\left\{\mathbf{y}_{q}[k] \mathbf{y}_{q}^{H}[k]\right\}$ and the pseudo-autocorrelation $\mathbb{E}\left\{\mathbf{y}_{q}[k] \mathbf{y}_{q}^{T}[k]\right\}$ are needed to fully characterize the secondorder statistics of $\mathbf{y}_{q}[k]$. When non-circularity appears, the best linear procedure of estimation consists in linearly combining $\mathbf{y}_{q}[k]$ and $\mathbf{y}_{q}^{*}[k]$, which is known by the name of widely linear filtering [56]. When the parameters to be estimated are realvalued, as it is the case when the PAM modulation comes into play, the widely linear filtering is equivalent to linearly combining $\Re\left(\mathbf{y}_{q}[k]\right)$ and $\Im\left(\mathbf{y}_{q}[k]\right)$. Therefore, all the information will be retrieved by any of these two augmented vectors, namely $\left[\begin{array}{ll}\mathbf{y}_{q}^{T}[k] & \mathbf{y}_{q}^{H}[k]\end{array}\right]^{T}$ or $\left[\Re\left(\mathbf{y}_{q}^{T}[k]\right) \quad \Im\left(\mathbf{y}_{q}^{T}[k]\right)\right]^{T}$. Due to the special processing that is required to detect non-circular symbols, an open problem is to devise techniques that take full advantage of this fact.

\section{E. Scenarios}

As it was argued in previous subsections, the simplification of the input/output relation depends on how severe the frequency selectivity of the channel is. Three scenarios will be considered, corresponding to different degrees of channel frequency selectivity. They are described in Table II, where the corresponding models from (26) that are valid in each case are also shown. The parameters that characterize Scenarios 2 and 3 allow approximating the channel frequency response as flat within one subchannel. Therefore, in this case, model 1) can be replaced by model 2). In Scenario 1, no flatness assumption can be made and, therefore, model 1) is the only input/output relation that can accurately represent the equivalent channel. Concerning the dynamics of the channel, the paper is restricted to scenarios where the channel is invariant over several FBMC/OQAM symbols.

In all three scenarios the FBMC/OQAM signal is constructed with the prototype pulse presented in [57] with an overlapping factor $K=4$. To make a fair comparison between FBMC/OQAM and OFDM, the symbol energy to noise ratio 
Table II

SYSTEM PARAMETERS AND PROPAGATION CONDITIONS

\begin{tabular}{|c|c|c|c|}
\hline & Scenario 1 & Scenario 2 & Scenario 3 \\
\hline Subcarrier spacing & $\Delta_{f}=15 \mathrm{kHz}$ & $\Delta_{f}=15 \mathrm{kHz}$ & $\Delta_{f}=15 \mathrm{kHz}$ \\
\hline Sampling frequency & $f_{s}=M \Delta_{f} \mathrm{~Hz}$ & $f_{s}=M \Delta_{f} \mathrm{~Hz}$ & $f_{s}=M \Delta_{f} \mathrm{~Hz}$ \\
\hline Channel model [53] & Extended Typical Urban (ETU) & Extended Vehicular A (EVA) & Extended Pedestrian A (EPA) \\
\hline System model & $1)$ & $1), 2)$ & $1), 2)$ \\
\hline
\end{tabular}

loss, due to the $\mathrm{CP}$ transmission, has been taken into account in all the simulations. The length of the $\mathrm{CP}$ is set to $\frac{1}{8}$ of the symbol period, unless otherwise stated.

\section{F. Robustness against synchronization errors}

One of the main advantages of FBMC/OQAM over OFDM is its inherent robustness against synchronization errors. In fact, among the main interests in the FBMC/OQAM technology is the fact that it enables the practical transmission of several (unsynchronized) signals occupying different subcarrier groups. Contrary to OFDMA, where strict signal synchronization among the different users is needed, the synchronization requirements between the distinct multi-user FBMC/OQAM signals can be relaxed. This was recently demonstrated in [58], where an exhaustive analytical study of the residual distortion caused by multi-user misalignment was presented. It was concluded that the performance degradation from synchronization errors is negligible provided that at least one subcarrier is left void between adjacent multiuser transmissions. The same conclusion has been drawn in [59]. In OFDMA, a single subcarrier does not suffice to separate the signals from different users. At the price of a complexity increase, the sensitivity to synchronization errors can be reduced by resorting to interference mitigation techniques [59], [60]. Section IV-C4 further delves into the robustness against synchronization errors, by carrying out a performance evaluation of FBMC/OQAM and OFDM when tight synchronization is not attained.

\section{MIMO-FBMC/OQAM SYSTEMS WITHOUT CSIT REQUIREMENTS: SPATIAL MULTIPLEXING SCHEMES}

This section presents the techniques that have been specifically designed for MIMO-FBMC/OQAM systems when CSI is solely available at the receiver. Emphasis is given to schemes that achieve spatial multiplexing gains, where $N_{T}$ streams are plainly mapped onto $N_{T}$ transmit antennas. In this case the streams can be detected jointly, or separately after performing MIMO equalization. Depending on how strong the channel frequency selectivity is, this section proposes specific techniques for highly and low frequency selective channels.

When CSI is only exploited by the receiver, diversity gains can be achieved using space-time-block-coding (STBC) [61]. Nevertheless, the PR property in (10) is satisfied in the real domain, whereas STBC is constructed by using orthogonal structures in the complex field. As a consequence, the application of STBC to FBMC/OQAM results in ISI and ICI [62]. To overcome the inherent error floor problem, ISI and ICI can be mitigated by interference estimation and cancellation procedures [63], [64]. This allows achieving diversity gains, but only for low-order modulations. Indeed, interference estimation and cancellation techniques suffer from error propagation phenomenon. When high-order modulations are used, the decision errors occur more often and propagate through the iterations. Therefore, the performance still presents the error floor limitation for high-order modulations. In addition, the best performance in [63], [64] is achieved by using QAM symbols with the conventional FT lattice structure of OFDM.

Alternatively, STBC can be combined with FBMC/OQAM in a block-wise manner [65]. This solution is feasible when the FBMC/OQAM transmultiplexer impulse response is conjugate symmetric along the time axis. The Alamouti scheme can benefit from this property to create the complex conjugate symbols to be transmitted in the second slot, by transmitting the time-reversal version of the corresponding block. Then, the conventional Alamouti decoding scheme is able to separate the streams in the FBMC/OQAM context as well. However, zero-valued symbols have to be inserted between the blocks to avoid the interference between them.

Other research works propose to modify the FBMC/OQAM scheme to enable the combination with STBC [62], [66], [67]. The authors in [62] show that Alamouti coding can be employed when it is combined with code division multiple access (CDMA). The scheme proposed in [66] avoids the error floor problem, by grouping consecutive FBMC/OQAM signals and appending a $\mathrm{CP}$ ahead of each block. When the $\mathrm{CP}$ is removed at the receive side, the channel matrix that multiplies each block can be easily diagonalized, which paves the way to applying the Alamouti scheme. Another technique that relies on the CP transmission is developed in [67]. In contrast to [66], the CP is inserted on each subcarrier at the input of the SFB. Then, ISI can be eliminated by applying channel diagonalization strategies on each subcarrier. To remove ICI, adjacent subcarriers transmit complex data in different FT positions, which allows combining STBC and FBMC/OQAM in a straightforward manner. Since the aforementioned techniques are not based on conventional FBMC/OQAM schemes and achieve diversity gains at the expense of higher complexity and rate degradation, the development of MIMO techniques that achieve diversity is not covered in this section.

\section{A. Highly frequency selective channels}

Starting from the general expression in (25), which considers the model in highly frequency selective channels, the demodulated signal in the absence of CSIT can be expressed as

$$
\begin{aligned}
\mathbf{y}_{q}[k] & =\theta_{q}[k] \mathbf{G}_{q q}[0] \mathbf{d}_{q}[k]+\mathbf{w}_{q}[k] \\
& +\sum_{(m, \tau) \in \Omega_{q, k}} \theta_{m}[k-\tau] \mathbf{G}_{q m}[\tau] \mathbf{d}_{m}[k-\tau] .
\end{aligned}
$$


Observe that symbols are not precoded, so that $\mathbf{v}_{q}[k]=$ $\theta_{q}[k] \mathbf{d}_{q}[k]$. This equation shows that the time-frequency signaling in FBMC/OQAM leads to two-dimensional (2-D) interference. To produce maximum likelihood (ML) sequence estimates, it is necessary to construct a trellis that characterizes the 2-D interference. Since the number of states of the trellis grows exponentially with $N_{T}$ and the number of elements in the subset $\Omega_{q, k}$, one can conclude that ML sequence estimation is computationally intractable. Several suboptimal trellisbased detection algorithms are surveyed in [68]. However, the required complexity is still too high in the context of MIMOFBMC/OQAM. Hence, the rest of the subsection delves into equalization-aided receivers that perform separate detection. The most promising techniques fall into three categories, which are identified as parallel equalization, multi-tap equalization and frequency-domain equalization.

1) Parallel equalization: As mentioned in Section II-B, the most general multi-antenna linear transceiver for frequency selective channels can be described as in Fig. 5. When the signal is transmitted without precoding, one could build the frequency selective equalizer matrix $\mathbf{A}(\omega)$ according to the zero forcing $(\mathrm{ZF})$, i.e.,

$$
\mathbf{A}^{H}(\omega) \mathbf{H}(\omega)=\mathbf{I}_{S},
$$

or the minimum mean square error (MMSE) criteria, yielding

$$
\mathbf{A}(\omega)=\left(\mathbf{H}(\omega) \mathbf{H}^{H}(\omega)+\frac{N_{0}}{E_{S}} \mathbf{I}_{N_{R}}\right)^{-1} \mathbf{H}^{H}(\omega) .
$$

Since these two approaches are not easily affordable from the computational point of view, it is customary to exploit the FBMC/OQAM signal structure in order to simplify the implementation of these frequency-selective receiving structures.

To illustrate this point, let us focus on this ideal equalizer structure for some specific symbol stream. The ideal receiver is described by the concatenation of the frequency selective equalizer $\mathbf{A}(\omega)$ and the AFB. Therefore, from the structure of the AFB in Fig. 4 we can readily see the frequency response observed by the $m$ th subcarrier prior to decimation is given by the matrix $\left(F_{m}(\omega) \mathbf{A}(\omega)\right)^{H}$, where $F_{m}(\omega)$ is defined in (19). The prototype pulse $p[n]$ is typically designed to occupy a relatively narrow bandwidth, which means that the energy of the output of the filter $F_{m}(\omega)$ will be mostly concentrated around $\omega=\frac{2 \pi m}{M}$. As a consequence, if we consider the concatenation of the equalizer matrix $\mathbf{A}(\omega)$ and the AFB, we may approximate

$$
F_{m}(\omega) \mathbf{A}(\omega) \simeq F_{m}(\omega) \mathbf{A}\left(\frac{2 \pi m}{M}\right)=F_{m}(\omega) \mathbf{A}_{m}
$$

under the assumption that the entries of $\mathbf{A}(\omega)$ do not present strong variations around the subcarrier $\omega=\frac{2 \pi m}{M}$. In this case, the general receive architecture shown in Fig. 5 can essentially be implemented with the structure illustrated in Fig. 6. We will next see that more accurate approximations can be considered, which will effectively deal with strong channel frequency selectivity. The idea is simply to consider a more accurate approximation of $\mathbf{A}(\omega)$ around the intended subcarrier.
Assume that the equalizer matrix $\mathbf{A}(\omega)$ is a continuously differentiable function of order up to $K_{R}$ (the subindex $R$ here emphasizing the fact that we are considering a receive architecture) and denote by $\mathbf{A}^{(\ell)}(\omega)$ its $\ell$ th derivative. Using a Taylor expansion, we can approximate the original equalizer $\mathbf{A}(\omega)$ around the $m$ th subcarrier as

$$
\mathbf{A}(\omega) \simeq \sum_{\ell=0}^{K_{R}-1} \frac{1}{\ell !}\left(\omega-\frac{2 \pi m}{M}\right)^{\ell} \mathbf{A}_{m}^{(\ell)}
$$

where $\mathbf{A}_{m}^{(\ell)}=\mathbf{A}^{(\ell)}\left(\frac{2 \pi m}{M}\right)$. Now, consider again the concatenation of the equalizer and the AFB. We can clearly approximate

$$
F_{m}(\omega) \mathbf{A}(\omega) \simeq \sum_{\ell=0}^{K_{R}-1} \frac{1}{\ell !}\left(\omega-\frac{2 \pi m}{M}\right)^{\ell} F_{m}(\omega) \mathbf{A}_{m}^{(\ell)}
$$

This approximation is more accurate than the one in (34), which can be retrieved from the above by selecting $K_{R}=$ 1. Now, the right hand side of (36) can be very efficiently implemented by using $K_{R}$ parallel filterbank demodulators, each one of them constructed from a different prototype pulse. Indeed, observe that the frequency response in (36) can be implemented as the sum of $K_{R}$ parallel frequency responses, each one obtained as the concatenation of a frequency-flat equalizer $\mathbf{A}_{m}^{(\ell)}$ and a filter with frequency response

$$
\begin{aligned}
\frac{1}{\ell !}(\omega & \left.-\frac{2 \pi m}{M}\right)^{\ell} F_{m}(\omega)= \\
& =\frac{e^{-j \frac{2 \pi}{M} m D}}{\ell !}\left(\omega-\frac{2 \pi m}{M}\right)^{\ell} P\left(\omega-\frac{2 \pi m}{M}\right) .
\end{aligned}
$$

The frequency response of this filter is proportional to a frequency-translated version of $\omega^{\ell} P(\omega)$, which can approximately be seen as the DTFT of the time-domain derivative of the prototype pulse.

In order to formalize this point, we recall that according to Section II-A, the prototype pulse $p[n]$ can be obtained as a discretization of a smooth analog waveform $p(t)$ : $\left[-\left(L T_{s}\right) / 2,\left(L T_{s}\right) / 2\right] \rightarrow \mathbb{R}$, so that

$$
p[n]=p\left((n-D) T_{s}\right), \quad n=0, \ldots, L-1 .
$$

Let us denote by $p^{(\ell)}(t)$ the $\ell$ th derivative of the analog waveform $p(t)$. Define $p^{(\ell)}[n]$ as the corresponding discretization, i.e.,

$$
p^{(\ell)}[n]=\left(M T_{s}\right)^{\ell} p^{(\ell)}\left((n-D) T_{s}\right)
$$

and let $P_{\ell}(\omega)$ denote its DTFT. Then, we can easily see that $P_{\ell}(\omega) \simeq(j \omega M)^{\ell} P(\omega)$ for sufficiently large $M$. Indeed, this relation only holds true for analog signals. However, if $M$ is high enough, the energy of $P(\omega)$ becomes so concentrated around the origin that frequency aliasing tends to disappear and consequently, the DTFT and the continuous-time Fourier transform become proportional. Hence, we may re-write the approximation in (36) as

$$
F_{m}(\omega) \mathbf{A}(\omega) \simeq \sum_{\ell=0}^{K_{R}-1} \frac{1}{\ell !(j M)^{\ell}} F_{\ell, m}(\omega) \mathbf{A}_{m}^{(\ell)}
$$




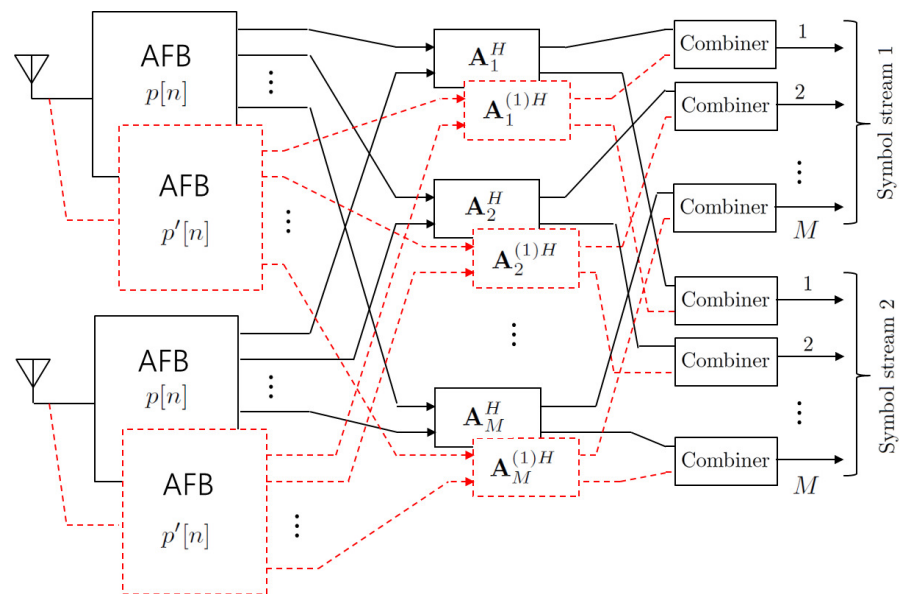

Figure 8. Proposed implementation of the frequency-selective linear receiver (equalizer) for the specific case of $N_{R}=2$ receive antennas and $K_{R}=2$ parallel stages.

where $F_{\ell, m}(\omega)=e^{-j \frac{2 \pi}{M} m D} P_{\ell}\left(\omega-\frac{2 \pi m}{M}\right)$. According to this equation, the optimum equalizer can be approximated by properly combining $K_{R}$ parallel frequency flat equalizer matrices $\mathbf{A}_{m}^{(\ell)}$, taken as the derivatives of the original $\mathbf{A}(\omega)$ at each subcarrier $m=1, \ldots, M$. Each frequency flat equalizer matrix $\mathbf{A}_{m}^{(\ell)}$ feeds an FBMC/OQAM modulator whose frequency response at the $m$ th subcarrier is given by $F_{\ell, m}(\omega)$, which corresponds to a classical FBMC/OQAM modulator that uses a derivative prototype $p^{(\ell)}[n]$ as given in (39) instead of the original one $p[n]$.

In summary, we can conclude by the above informal reasoning that the ideal targeted equalizer formulated in (32) and (33) can be approximated as illustrated in Fig. 8. The suggested implementation of the multi-stage equalizer is represented for the specific case where $K_{R}=2$ (2 parallel stages) and $N_{R}=2$ (two receive antennas). The additional stage that needs to be superposed to the original one is represented in red dotted line.

The performance of this parallel multi-stage equalizer was established in [69] by using the steps drawn in [70] for the SISO case. In particular, it was shown in [69] that, under PR pulse conditions, the distortion at the output of the equalizer decays as $O\left(M^{-2 K_{R}}\right)$ when $M \rightarrow \infty$, where $K_{R}$ is the number of parallel receive stages. Thus, by increasing $K_{R}$, one can progressively reduce the residual distortion caused by the channel frequency selectivity. In order to establish how many stages are needed to achieve a certain performance level, one can evaluate an analytic expression of the asymptotic residual error power (see [69] for further details).

2) Multi-tap equalization: The use of multi-tap equalization in conventional FBMC/OQAM architectures raises as an alternative to parallel equalization to face the channel frequency selectivity. The effectiveness of multi-tap equalization in SISO communication systems is corroborated by the work in [71], [72]. In this subsection, the same idea is extended to the spatial multiplexing case [73], [74]. In this regard, the demodulated signal is equalized resorting to space and time diversity, giving rise to

$$
\mathbf{z}_{q}[k]=\sum_{\tau=-L_{a}}^{L_{a}} \mathbf{A}_{q}^{H}[\tau] \mathbf{y}_{q}[k-\tau] .
$$

By design, $\mathbf{A}_{q}[k] \in \mathbb{C}^{N_{R} \times S}$ is assumed different from 0 for $-L_{a} \leq k \leq L_{a}$. Since the transmitted symbols only bear useful information in either the real or the imaginary dimensions, we can compensate the phase term and focus on the real part, leading to $\check{\mathbf{d}}_{q}[k]=\Re\left(\theta_{q}^{*}[k] \mathbf{z}_{q}[k]\right)$. To get easy-tohandle expressions, the estimated symbols can be compactly expressed using this formulation

$$
\begin{aligned}
\check{\mathbf{d}}_{q}[k]= & \overline{\mathbf{A}}_{q}^{T} \overline{\mathbf{E}}_{q q}^{k}[0] \mathbf{d}_{q}[k]+\overline{\mathbf{A}}_{q}^{T} \overline{\boldsymbol{\eta}}_{q}[k] \\
& +\sum_{(m, \tau) \in \Omega_{q, k}} \overline{\mathbf{A}}_{q}^{T} \overline{\mathbf{E}}_{q m}^{k}[\tau] \mathbf{d}_{m}[k-\tau],
\end{aligned}
$$

by stacking real and imaginary parts of these matrices

$$
\begin{aligned}
\mathbf{A}_{q}= & {\left[\mathbf{A}_{q}^{T}\left[-L_{a}\right] \cdots \mathbf{A}_{q}^{T}\left[L_{a}\right]\right]^{T} } \\
\boldsymbol{\eta}_{q}[k]= & \theta_{q}^{*}[k]\left[\mathbf{w}_{q}\left[k+L_{a}\right] \cdots \mathbf{w}_{q}\left[k-L_{a}\right]\right]^{T} \\
\mathbf{E}_{q m}^{k}[\tau]= & \theta_{q}^{*}[k] \theta_{m}[k-\tau]\left[\mathbf{G}_{q m}^{T}\left[\tau+L_{a}\right]\right. \\
& \left.\cdots \mathbf{G}_{q m}^{T}\left[\tau-L_{a}\right]\right]^{T} .
\end{aligned}
$$

The real-valued representation relies on the notation described in Section I. From this point on, the derivation of the widely linear MMSE receiver is the closed-form expression given by

$$
\overline{\mathbf{A}}_{q}=\left(\sum_{m, \tau} \overline{\mathbf{E}}_{q m}^{k}[\tau]\left(\overline{\mathbf{E}}_{q m}^{k}[\tau]\right)^{T}+\frac{1}{E_{S}} \mathbf{R}_{\eta_{q}}\right)^{-1} \overline{\mathbf{E}}_{q q}^{k}[0]
$$

The noise autocorrelation matrix is denoted by $\mathbf{R}_{\eta_{q}}=$ $\mathbb{E}\left\{\overline{\boldsymbol{\eta}}_{q}[k] \overline{\boldsymbol{\eta}}_{q}^{T}[k]\right\}$. In order to improve the performance, the MMSE receiver can be combined with successive interference cancellation (SIC) structures [73]. Due to the matrix inversion, the complexity involved in the computation of MMSE-based techniques is high, for large $N_{T}$ and $N_{R}$. The number of operations required to calculate the equalizer coefficients can be significantly reduced if the frequency sampling approach derived in [74] is considered. Frequency sampling-based equalizers are designed to match a target frequency response at a given frequency points, therefore they can be efficiently computed resorting to the discrete Fourier transform (DFT).

3) Frequency-domain equalization: With the multi-tap approach, a processing delay is introduced to leverage on the temporal diversity. To combat the effects of non-flat subchannels without additional delays, an alternative scheme named frequency spreading FBMC/OQAM (FS-FBMC/OQAM) is presented in [75]. The method is based on designing the frequency response of the prototype pulse, which is denoted by $P(\omega)$, according to the approach in [57]. Then, for an overlapping factor $K$, the evaluation of $P(\omega)$ at the frequency spacing $\frac{2 \pi}{K M}$ is different from zero only in $2 K-1$ points, i.e.,

$$
P\left(\frac{2 \pi i}{K M}\right) \neq 0, \quad i=-K+1, \cdots, K-1 .
$$


$r_{j}[k M / 2] \cdots r_{j}[k M / 2+K M-1]$

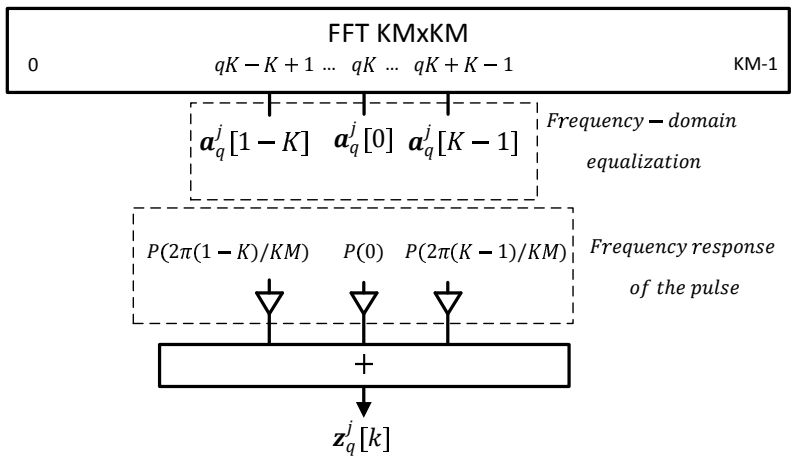

Figure 9. Frequency-domain equalizer for subcarrier $q$ and antenna $j$.

To benefit from the fact that the pulse is only defined by $2 K-1$ non-zero frequency components, the AFB can be easily implemented in the frequency domain. Assuming that the length of the pulse in the time domain is $K M$ and denoting by $r_{j}[n]$ the signal received by the $j$ th antenna, the $q$ th output of the AFB is written as

$$
y_{q}^{j}[k]=\sum_{n=0}^{K M-1} r_{j}\left[n+k \frac{M}{2}\right] p[n] e^{-j \frac{2 \pi}{M} q(n-D)} .
$$

Leveraging on the inverse DFT of the pulse, given by

$$
p[n]=\frac{1}{K M} \sum_{i=-K+1}^{K-1} P\left(\frac{2 \pi i}{K M}\right) e^{j \frac{2 \pi}{K M} i n},
$$

the demodulated signal can be expressed as

$$
y_{q}^{j}[k]=\frac{e^{j \frac{2 \pi q D}{M}}}{K M} \sum_{i=-K+1}^{K-1} R_{j k}\left(\frac{2 \pi(q K+i)}{K M}\right) P\left(\frac{2 \pi i}{K M}\right)
$$

where

$$
R_{k}^{j}\left(\frac{2 \pi}{K M} l\right)=\sum_{n=0}^{K M-1} r_{j}\left[n+k \frac{M}{2}\right] e^{-j \frac{2 \pi}{K M} n l} .
$$

It must be noted that (48) can be efficiently implemented with a $K M \times K M$ FFT. However, the output of the FFT is not the point-wise multiplication of the channel and the transmitted signal frequency responses. To prove it, let us define

$$
S_{k}^{i}\left(\frac{2 \pi}{K M} l\right)=\sum_{n=0}^{K M-1} s_{i}\left[n+k \frac{M}{2}\right] e^{-j \frac{2 \pi}{K M} n l},
$$

where $s_{i}[n]$ is the signal transmitted by the $i$ th antenna. Neglecting the noise, the signal received by the $j$ th antenna takes the form $r_{j}[n]=\sum_{i=1}^{N_{T}} s_{i}[n] * h_{j i}[n]$. Denoting the DTFT of $h_{j i}[n]$ by $H_{j i}(\omega)$, the following relation

$$
R_{k}^{j}\left(\frac{2 \pi}{K M} l\right)=\sum_{i=1}^{N_{T}} S_{k}^{i}\left(\frac{2 \pi}{K M} l\right) H_{j i}\left(\frac{2 \pi}{K M} l\right)
$$

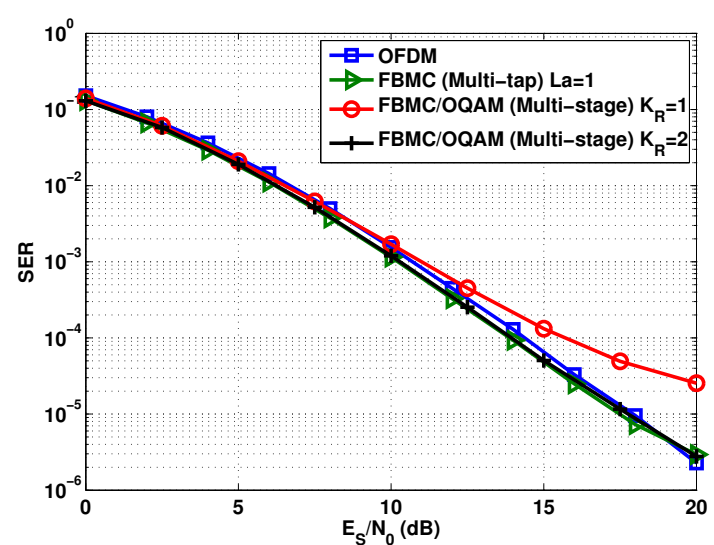

Figure 10. SER against $\frac{E_{S}}{N_{0}}$ in highly frequency selective channels.

cannot be assumed as the true expression when the FFT is applied. The explanation is based on realizing that the linear convolution of the sequence $s_{i}\left[k \frac{M}{2}\right] \cdots s_{i}\left[k \frac{M}{2}+K M-1\right]$ with the channel, results in a signal the length of which is higher than $K M$. Provided that the length of the channel was $L_{c h}$, the FFT size should be at least $K M+L_{c h}-1$ to achieve the desired point-wise multiplication [76]. Then, the DFT of $p[n]$ may not be defined by $2 K-1$ points, significantly increasing the complexity. Based on that, it is proposed to stick to the configuration described in [75] and assume that (51) is satisfied. However, further research is required to characterize the distortion caused by taking blocks of $K M$ samples.

As Fig. 9 indicates, the procedure to estimate $\mathbf{d}_{q}[k]$ consists in multiplying the $(q K+i)$ th FFT output by $\mathbf{a}_{q}^{j}[i] \in \mathbb{C}^{N_{T} \times 1}$, for $i=-K+1, \cdots, K-1$. Note that the size of the equalizer in the spatial multiplexing case is $N_{T}$. After the equalization stage, the signals are weighted with the DFT of $p[n]$. Finally, the resulting $2 K-1$ spectral components are added leading to the column vector $\mathbf{z}_{q}^{j}[k] \in \mathbb{C}^{N_{T} \times 1}$. Finally, the symbols can be estimated as

$$
\check{\mathbf{d}}_{q}[k]=\frac{1}{K M} \Re\left(\theta_{q}^{*}[k] e^{-j \frac{2 \pi q D}{M}} \sum_{j=1}^{N_{R}} \mathbf{z}_{q}^{j}[k]\right) .
$$

Provided that the MMSE is the equalization technique used to separate the streams, the taps are derived as follows [77]:

$$
\left[\mathbf{a}_{q}^{1}[i] \ldots \mathbf{a}_{q}^{N_{R}}[i]\right]=\left(\mathbf{H}_{q, i}^{H} \mathbf{H}_{q, i}+\frac{N_{0}}{E_{S}} \mathbf{I}_{N_{T}}\right)^{-1} \mathbf{H}_{q, i}^{H},
$$

where $\mathbf{H}_{q, i} \in \mathbb{C}^{N_{R} \times N_{T}}$ is the DTFT of the MIMO channel evaluated at $\frac{2 \pi}{K M}(q K+i)$.

4) Performance Validation: In order to validate the performance of the multi-stage architecture and the multi-tap processing, we simulate a FBMC/OQAM modulation with $M=1024$ subcarriers. The MIMO setup is constructed according to the scenario 1 , whose system parameters are defined in Table II, with $N_{T}=2$ and $N_{R}=4$. The symbols are drawn from the 16-QAM constellation. Regarding the multistage architecture, the original equalizer is designed according 
to (33). The solution based on the multi-tap processing follows the approach described in Section III-A2. In OFDM, which is simulated as a benchmark, the channel is equalized with the MMSE receiver.

Fig. 10 represents the symbol error rate (SER) as a function of $\frac{E_{S}}{N_{0}}$. Observe that FBMC/OQAM exhibits an error floor when the multi-stage parallel architecture sets $K_{R}=1$, which coincides with the conventional single-tap MMSE equalizer employed in OFDM. When the receiver combines the processing performed on two stages, which corresponds to $K_{R}=2$, the error floor is not observable for the range of $\frac{E_{S}}{N_{0}}$ under consideration. The alternative based on multi-tap equalization gives practically the same performance as the multi-stage architecture by using three taps per-subcarrier, i.e. $L_{a}=1$. Notice that the curves obtained in OFDM and FBMC/OQAM systems exhibit the same slope. However, there is a gap due to the energy that is wasted transmitting the CP in OFDM.

The BER results of the multi-tap equalizer virtually coincide with those obtained with the frequency sampling approach and the frequency-domain equalizer, which are respectively descried in Sections III-A2 and III-A3. For the sake of the clarity in the presentation, the aforementioned techniques have not been represented in Fig. 10.

\section{B. Low frequency selective channels}

Considering frequency non-selective subchannels, the vector of the demodulated signals at a given FT position $(q, k)$ is expressed as

$$
\begin{aligned}
\mathbf{y}_{q}[k] & =\theta_{q}[k] \mathbf{H}_{q} \mathbf{d}_{q}[k]+\mathbf{w}_{q}[k] \\
& +\sum_{(m, \tau) \in \Omega_{q, k}} \alpha_{q m}[\tau] \theta_{m}[k-\tau] \mathbf{H}_{q} \mathbf{d}_{m}[k-\tau] .
\end{aligned}
$$

It has been assumed that the channel seen by all the signals that leak through the $q$ th subcarrier is the same and equal to $\mathbf{H}_{q}$, which can be considered as a special case of model 2). It will be shown that the assumption made in (54) paves the way to applying ML detection. To this end, $\mathbf{y}_{q}[k]$ has to be further processed before being fed into the detector. In this regard, the proposed strategies resort to equalization and interference cancellation (IC).

1) Single-tap equalization: The key point to perform equalization in low frequency selective channels stems from realizing that (54), after phase compensation, can be compactly expressed as

$$
\mathbf{r}_{q}[k]=\theta_{q}^{*}[k] \mathbf{y}_{q}[k]=\mathbf{H}_{q}\left(\mathbf{d}_{q}[k]+j \mathbf{u}_{q}[k]\right)+\theta_{q}^{*}[k] \mathbf{w}_{q}[k] .
$$

The interference symbol is represented as

$$
j \mathbf{u}_{q}[k]=\sum_{(m, \tau) \in \Omega_{q, k}} \Gamma_{q m}^{k}[\tau] \mathbf{d}_{m}[k-\tau],
$$

where $\Gamma_{q m}^{k}[\tau]$ is defined in (11). It has been assumed that the pulses fulfill the PR property (10). Then, it can be readily verified that the interference symbol is pure imaginary. When considering (55), linear equalization designed on the basis of ZF and MMSE criteria, can be straightforwardly applied as described in [78]. The design is drastically simplified, if the equalization matrix $\mathbf{A}_{q} \in \mathbb{C}^{N_{R} \times N_{T}}$ targets the virtually transmitted vector

$$
\mathbf{c}_{q}[k]=\mathbf{d}_{q}[k]+j \mathbf{u}_{q}[k],
$$

rather than $\mathbf{d}_{q}[k]$. The autocorrelation matrix of the interference symbol can be approximated by $\mathbb{E}\left\{\mathbf{u}_{q}[k] \mathbf{u}_{q}^{H}[k]\right\} \approx$ $E_{S} \mathbf{I}_{N_{T}}$, by using the values of Table I. Then, it follows that $\mathbb{E}\left\{\mathbf{c}_{q}[k] \mathbf{c}_{q}^{H}[k]\right\} \approx 2 E_{S} \mathbf{I}_{N_{T}}$ and, thus, the MMSE equalizer becomes

$$
\mathbf{A}_{q}=\left(\mathbf{H}_{q} \mathbf{H}_{q}^{H}+\frac{N_{0}}{2 E_{S}} \mathbf{I}_{N_{R}}\right)^{-1} \mathbf{H}_{q}^{H} .
$$

The equalized symbol is

$$
\check{\mathbf{c}}_{q}[k]=\mathbf{A}_{q}^{H} \mathbf{r}_{q}[k],
$$

and its real part yields the equalized data vector

$$
\check{\mathbf{d}}_{q}[k]=\Re\left(\check{\mathbf{c}}_{q}[k]\right)=\frac{1}{2}\left(\mathbf{A}_{q}^{H} \mathbf{r}_{q}[k]+\mathbf{A}_{q}^{T} \mathbf{r}_{q}^{*}[k]\right) .
$$

Interestingly, it can be checked that the strategy based on performing linear equalization and extracting the real part, coincides with the widely linear MMSE receiver. It must be mentioned that this statement does not hold true if $\mathbb{E}\left\{\mathbf{u}_{q}[k] \mathbf{u}_{q}^{H}[k]\right\} \neq \mathbb{E}\left\{\mathbf{d}_{q}[k] \mathbf{d}_{q}^{T}[k]\right\}$.

Finally, taking for granted that the residual interference is negligible after equalization, symbols can be independently detected.

2) Full interference cancellation: An attempt to outperform single-tap equalization is based on estimating the intrinsic interference and then, perform interference cancellation, to either apply ML detection [79] or exploit widely linear processing [80] afterward. Building upon the linear equalizer formulated in (58), two ways have been established to estimate the intrinsic interference. According to subsection III-B1, the MMSE equalizer provides an estimation of the virtual transmitted symbol vector $\mathbf{c}_{q}[k]=\mathbf{d}_{q}[k]+j \mathbf{u}_{q}[k]$. Therefore, an evaluation of the interference is available by taking only the imaginary part of (59), i.e.,

$$
j \check{\mathbf{u}}_{q}[k]=j \Im\left(\check{\mathbf{c}}_{q}[k]\right) .
$$

Via a reconstruction process of the already detected symbols, the intrinsic interference can be alternatively obtained as

$$
j \check{\mathbf{u}}_{q}[k]=\sum_{(m, \tau) \in \Omega_{q, k}} \Gamma_{q m}^{k}[\tau] \mathbf{d}_{m}^{0}[k-\tau] .
$$

The tentative symbols $\left\{\mathbf{d}_{q}^{0}[k]\right\}$ are the estimates of $\left\{\mathbf{d}_{q}[k]\right\}$ given in (60). Although the second option introduces a processing delay, it provides more reliable estimates than the first one. Once the interference is reconstructed, by any of the two possible ways, the unwanted contribution from the received vector is canceled. Then, the vector $\mathbf{z}_{q}[k]=\mathbf{r}_{q}[k]-j \mathbf{H}_{q} \check{\mathbf{u}}_{q}[k]$ is expressed as

$$
\mathbf{z}_{q}[k]=\mathbf{H}_{q}\left(\mathbf{d}_{q}[k]+j \boldsymbol{\epsilon}_{q}[k]\right)+\theta_{q}^{*}[k] \mathbf{w}_{q}[k],
$$




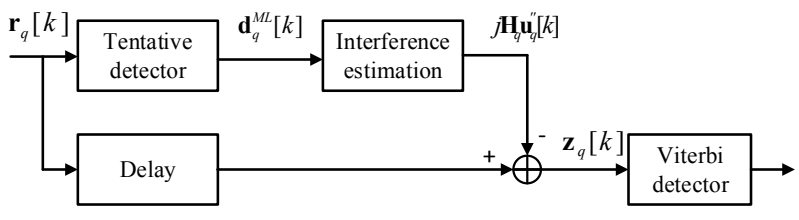

Figure 11. General receiver structure

where $\boldsymbol{\epsilon}_{q}[k]=\mathbf{u}_{q}[k]-\check{\mathbf{u}}_{q}[k]$ is the interference estimation error. Assuming perfect estimation, that is $\boldsymbol{\epsilon}_{q}[k]=\mathbf{0}$, the vector at the output of the interference cancellation stage is recast as

$$
\mathbf{z}_{q}[k]=\mathbf{H}_{q} \mathbf{d}_{q}[k]+\theta_{q}^{*}[k] \mathbf{w}_{q}[k] .
$$

Now, conventional ML detection can be applied with a complexity order $\mathcal{O}\left(M_{s}^{N_{T}}\right)$, where $M_{s}$ is the size of the symbol alphabet. Alternatively, a widely linear MMSE receiver can be employed on $\mathbf{z}_{q}[k]$ and $\mathbf{z}_{q}^{*}[k]$. The desired signal is accordingly expressed as

$$
\check{\mathbf{d}}_{q}[k]=\frac{1}{2}\left(\mathbf{A}_{q 1}^{H} \mathbf{z}_{q}[k]+\mathbf{A}_{q 2}^{H} \mathbf{z}_{q}^{*}[k]\right)
$$

where,

$$
\begin{aligned}
{\left[\begin{array}{ll}
\mathbf{A}_{q 1}^{H} & \mathbf{A}_{q 2}^{H}
\end{array}\right]=} & {\left[\begin{array}{ll}
\mathbf{H}_{q}^{H} & \mathbf{H}_{q}^{T}
\end{array}\right] \times } \\
& \left(\left[\begin{array}{cc}
\mathbf{H}_{q} \mathbf{H}_{q}^{H} & \mathbf{H}_{q} \mathbf{H}_{q}^{T} \\
\mathbf{H}_{q}^{*} \mathbf{H}_{q}^{H} & \mathbf{H}_{q}^{*} \mathbf{H}_{q}^{T}
\end{array}\right]+\frac{N_{0}}{E_{S}} \mathbf{I}_{2 N_{R}}\right)^{-1} .
\end{aligned}
$$

3) Partial interference cancellation: Full interference cancellation is effective only if the interference terms are sufficiently small. Unfortunately, the intrinsic interference and the desired symbol have almost the same power in the FBMC/OQAM context. Indeed. the example considered in the previous subsection satisfies $\mathbb{E}\left\{\mathbf{u}_{q}[k] \mathbf{u}_{q}^{H}[k]\right\} \approx$ $\mathbb{E}\left\{\mathbf{d}_{q}[k] \mathbf{d}_{q}^{T}[k]\right\}$. As a consequence, the error term in equation (63) will be non-zero at high and moderate noise regime. Hence, it can be stated that the error propagation is the main inhibitor to reach the optimal performance, when ML detectors and widely linear MMSE receivers are combined with full interference cancellation. To improve the performance with respect to the techniques addressed in subsection III-B1, partial interference cancellation (PaIC) is proposed in [81]. The receiver, which is shown in Fig. 11, is composed of a tentative detector that serves to partially cancel the interference, followed by a Viterbi detector. In this scheme, the set $\Omega_{q, k}$ is split into subsets $\Omega_{q, k}^{\prime}$ and $\Omega_{q, k}^{\prime \prime}$. Then, equation (55) becomes

$$
\begin{aligned}
\mathbf{r}_{q}[k] & =\mathbf{H}_{q}\left(\mathbf{d}_{q}[k]+\sum_{(m, \tau) \in \Omega_{q, k}^{\prime}} \mathbf{d}_{m}[k-\tau] \Gamma_{q m}^{k}[\tau]\right. \\
& \left.+\sum_{(m, \tau) \in \Omega_{q, k}^{\prime \prime}} \mathbf{d}_{m}[k-\tau] \Gamma_{q m}^{k}[\tau]\right)+\theta_{q}^{*}[k] \mathbf{w}_{q}[k] \\
& =\mathbf{H}_{q}\left(\mathbf{d}_{q}[k]+j \mathbf{u}_{q}^{\prime}[k]+j \mathbf{u}_{q}^{\prime \prime}[k]\right)+\theta_{q}^{*}[k] \mathbf{w}_{q}[k] .
\end{aligned}
$$

Analogously to previous subsection, the decided tentative estimates of (60), which are given by $\left\{\mathbf{d}_{q}^{0}[k]\right\}$, are utilized to reconstruct the interference associated to the subset $\Omega_{q, k}^{\prime \prime}$ as follows:

$$
j \check{\mathbf{u}}_{q}^{\prime \prime}[k]=\sum_{(m, \tau) \in \Omega_{q, k}^{\prime \prime}} \Gamma_{q m}^{k}[\tau] \mathbf{d}_{m}^{0}[k-\tau] .
$$

Assuming that the intrinsic interference resulting from the set $\Omega_{q, k}^{\prime \prime}$ has been completely removed, a Viterbi detector is then performed to match the non-canceled interference that comes from the remaining set $\Omega_{q, k}^{\prime}$. Depending on the set $\Omega_{q, k}^{\prime}$ a 2-D Viterbi detector can be required. However, designing a 2-D Viterbi is quite challenging and therefore, for simplicity reasons, it is recommended to limit the size of the set $\Omega_{q, k}^{\prime}$ in order to perform a 1-D Viterbi detection. In [81], the authors have determined that a satisfactory trade-off between complexity of the Viterbi detector and effectiveness of interference cancellation is achieved by the set

$$
\Omega_{q, k}^{\prime(1)}=\{(q, k-1) ;(q, k+1)\} .
$$

In this case, the autocorrelation matrix of $j \mathbf{u}_{q}^{\prime \prime}[k]$ is given by $0.3638 E_{s} \mathbf{I}_{N_{T}}$, using the values of Table I. After removing the interference and assuming perfect interference estimation, the vector $\mathbf{z}_{q}[k]=\mathbf{r}_{q}[k]-j \mathbf{H}_{q} \mathbf{u}_{q}^{\prime \prime}[k]$ is expressed as

$$
\begin{aligned}
\mathbf{z}_{q}[k]= & \mathbf{H}_{q}\left(\mathbf{d}_{q}[k-1] \Gamma_{q q}^{k}[-1]+\mathbf{d}_{q}[k]\right. \\
& \left.+\mathbf{d}_{q}[k+1] \Gamma_{q q}^{k}[1]\right)+\theta_{q}^{*}[k] \mathbf{w}_{q}[k] .
\end{aligned}
$$

Finally, $\mathbf{z}_{q}[k]$ is fed into the 1-D Viterbi detector.

4) Performance validation: We have compared the BER performance of the different receivers in a $2 \times 2$ spatial multiplexing system considering scenario 3 and channel model 3 ). The number of subcarriers is $M=1024$, and the data symbols are 4-QAM modulated. The considered receivers are the linear MMSE, the combination of MMSE with either ML detection or WL equalization and the PaIC. The receiver is referred to as MMSE-ML when ML estimation is applied after removing the interference by using the estimates provided in (61). By contrast, if the estimation of the interference relies on (62), then, the symbols can be jointly estimated according to the ML criterion or separately via WL filtering, once interference is eliminated. These two techniques are identified as IC-ML and IC-WL. The OFDM-ML receiver is given as a reference. In Fig. 12 the BER performance of these receivers is presented.

While the MMSE-ML receiver performs better than MMSE, we obtain an additional $1 \mathrm{~dB}$ performance gain when using both IC-ML and IC-WL. Those two receivers reach the same BER performance but the complexity of the second stage of the IC-WL is significantly lower than the IC-ML. However, the performance of IC-ML and IC-WL remains far from the one of OFDM-ML. This performance limitation is due to the reliability level of the MMSE equalizer. On the other hand, the $\mathrm{PaIC/Viterbi} \mathrm{scheme} \mathrm{outperforms} \mathrm{the} \mathrm{other} \mathrm{studied} \mathrm{schemes}$ and achieves almost the same performance as the OFDM-ML except at very high $\frac{E_{S}}{N_{0}}$. 


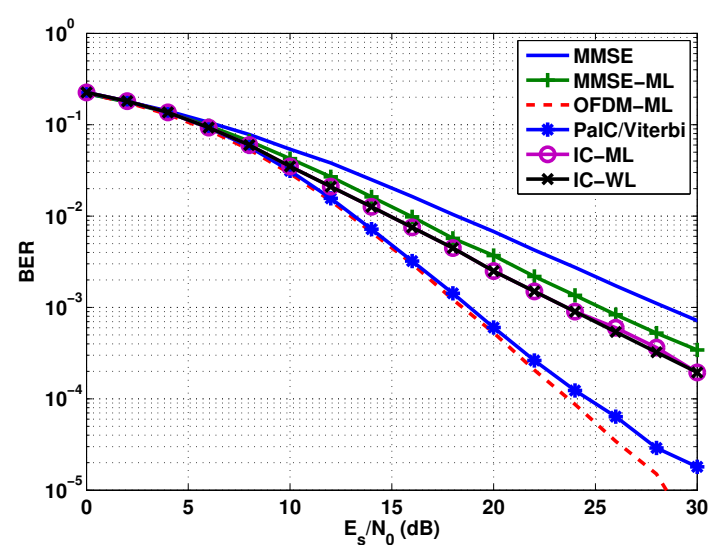

Figure 12. BER performance comparison of different interference cancellation receivers in a $2 \times 2$ spatial multiplexing system

\section{MIMO-FBMC/OQAM SySTEMS WITH CSIT}

The strategies covered in the subsequent sections allow for the joint design of the transmitter and the receiver due to the fact that CSI knowledge is available at both sides of the link. The possibility of using CSIT allows the transmitter to simultaneously serve several users using the same time/frequency resources, exploiting a space-division multiple access (SDMA) capability. In this sense, MIMO precoding and decoding matrix designs can be categorized into designs either for single- or for multi-user communication systems. This section also distinguishes between highly and low frequency selective subchannels and introduces the techniques that are more appropriate for each scenario. It is important to remark that the techniques addressed in this section are based on either the architecture depicted in Fig. 5 or the one in Fig. 6.

\section{A. Single-user communication systems. Highly frequency se- lective channels}

The first attempt to jointly design the transmit and the receive processing is limited to the study of single-user MIMO (SU-MIMO) communication systems. To solve to the highest possible extent the orthogonality issues when severe channel frequency selectivity comes into play, two different alternatives have been proposed, namely: multi-stage parallel processing and multi-tap processing.

1) Multi-stage parallel processing: The multi-stage equalization approach presented in Section III-A1 can be used in order to synthesize an approximation of the ideal precoding matrix $\mathbf{B}(\omega)$. Indeed, recalling that $F_{m}(\omega)$ denotes the DTFT of the $m$ th filter of the SFB, we can approximate the concatenation of $F_{m}(\omega)$ and the precoding matrix $\mathbf{B}(\omega)$ as

$$
F_{m}(\omega) \mathbf{B}(\omega) \simeq \sum_{\ell=0}^{K_{T}-1} \frac{1}{\ell !(j M)^{\ell}} F_{\ell, m}(\omega) \mathbf{B}_{m}^{(\ell)}
$$

for a certain $K_{T}$, where $\mathbf{B}_{m}^{(\ell)}=\mathbf{B}^{(\ell)}\left(\frac{2 \pi m}{M}\right)$ and where $F_{\ell, m}(\omega)$ is defined in Section III-A1. We recall here that $F_{\ell, m}(\omega)$ essentially corresponds to the DTFT of the $m$ th filter of the SFB constructed from the $\ell$ th time domain derivative of the prototype pulse $p[n]$.

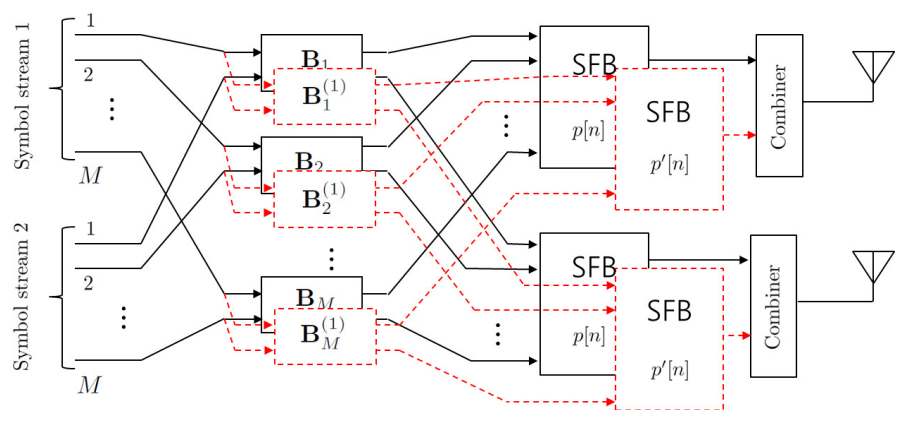

Figure 13. Proposed implementation of the frequecy-selective precoder for the specific case of $N_{T}=2$ transmit antennas and $N_{T}=2$ parallel stages.

Again, the optimum frequency selective precoder $\mathbf{B}(\omega)$ can be approximated by properly combining $K_{T}$ parallel frequency flat precoders $\mathbf{B}_{m}^{(\ell)}$, taken as the derivatives of the original $\mathbf{B}(\omega)$ at each subcarrier $m=0, \ldots, M-1$. Each frequency flat precoder matrix $\mathbf{B}_{m}^{(\ell)}$ feeds an FBMC modulator whose frequency response at the $m$ th subcarrier is given by $F_{\ell, m}(w)$, which corresponds to a classical FBMC modulator that uses a derivative prototype $p^{(\ell)}[n]$ as given in (39) instead of the original one $p[n]$.

The asymptotic performance (for large $M$ ) of the combination of a multi-stage precoder and linear receiver was established in [69]. Let $K_{T} \geq 1$ and $K_{R} \geq 1$ denote the number of parallel stages at the transmitter and the receiver respectively, and take $K_{\min }=\min \left(K_{T}, K_{R}\right)$. One can show that the distortion associated to the concatenation of such multi-stage precoder and linear receiver decays as $O\left(M^{-2 K_{\min }}\right)$ when $M \rightarrow \infty$. This means that the performance of the proposed system is asymptotically dictated by the minimum between the transmit and receive stages, i.e. $K_{\min }$. Therefore, it makes little sense to increase the number of parallel stages on one side of the link beyond the number of parallel stages on the other.

2) Multi-tap processing: We have seen that strong variations of the channel frequency response can be compensated through the parallel structure described in previous section. Following the same approach as subsection III-A2, frequency selectivity can be faced with multi-tap equalization. This subsection goes one step beyond and considers the use of a precoder in order to assist the equalizers. In this case, it is possible to draw an analogy between the partial subcarrier overlapping and the interference between users in multi-user communication systems. The concept is illustrated in Fig. 14. If symbols are precoded on a per-subcarrier basis, the precoding matrix $\mathbf{B} \in \mathbb{C}^{N_{T} M \times S M}$ becomes block diagonal and consequently, FBMC/OQAM bears a resemblance with the interference channel (IC). This similarity can be exploited when there is a power constraint for each subcarrier, by tailoring existing iterative methods that are used in the IC, e.g. [82], [83]. When symbols are jointly precoded we end up with a FBMC/OQAM scheme that is similar to a pointto-multipoint transmission in multi-user MIMO systems. This parallelism may allow us to benefit from the MSE-duality to design the transmitter and the receiver through alternating op- 


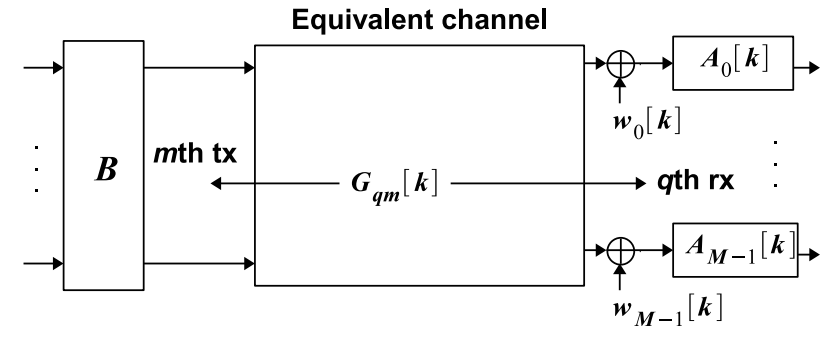

Figure 14. Analogy between FBMC/OQAM and single carrier multi-user communication systems.

timization algorithms [84]. Nonetheless, in the FBMC/OQAM context, the MSE-duality has only been investigated in multiuser single-input-multiple-output (SIMO) systems [85], so the MIMO case remains as an open problem. Fig. 14 stresses that if a global MIMO correlation matrix including all the subcarriers is generated, then it would be possible to express the input-output relation similarly to a narrowband MIMO system. Then, the SVD and the QR decompositions could be applied on the global MIMO matrix to either improve the capacity or facilitate the ML detection. Instead of exploiting the aforementioned analogies, which leads to complex solutions, this subsection focuses on finding closed-form solutions just for the case where $\mathbf{B}$ is block diagonal. Therefore, the symbols to be spatially multiplexed on the $m$ th subcarrier are precoded by the real-valued matrix $\mathbf{B}_{m} \in \mathbb{R}^{N_{T} \times S}$, yielding $\mathbf{v}_{m}[k]=\theta_{m}[k] \mathbf{B}_{m} \mathbf{d}_{m}[k]$. At the receive side, the broadband processing that allows to estimate the symbols can be expressed as $\mathbf{z}_{q}[k]=\sum_{\tau=-L_{a}}^{L_{a}} \mathbf{A}_{q}^{H}[\tau] \mathbf{y}_{q}[k-\tau]$, where generally $\mathbf{A}_{q}[k] \in \mathbb{C}^{N_{R} \times S}$ is different from 0 for $-L_{a} \leq k \leq L_{a}$. At this point, it is reasonable to question why precoders are real-valued. It has been experimentally verified that complex MIMO precoding matrices, result in much worse system performance when the technique addressed in this subsection is implemented. On the one hand, using real and imaginary dimensions keeps all the degrees of freedom. On the other hand, it becomes more challenging to suppress the interference that comes from the real and the imaginary domains. Since it has been experimentally observed that the impact of the latter overweights that of the former, the taps of $\left\{\mathbf{B}_{m}\right\}$ are constrained to be real-valued.

Finally, the transmitted data is estimated by extracting the information conveyed either in the real or the imaginary parts of the received samples, which boils down to operating as follows: $\check{\mathbf{d}}_{q}[k]=\Re\left(\theta_{q}^{*}[k] \mathbf{z}_{q}[k]\right)$. By plugging the precoders into (42), the estimated symbols can be expressed as

$$
\begin{aligned}
\check{\mathbf{d}}_{q}[k]= & \overline{\mathbf{A}}_{q}^{T} \overline{\mathbf{E}}_{q q}^{k}[0] \mathbf{B}_{q} \mathbf{d}_{q}[k]+\overline{\mathbf{A}}_{q}^{T} \overline{\boldsymbol{\eta}}_{q}[k] \\
& +\sum_{(m, \tau) \in \Omega_{q, k}} \overline{\mathbf{A}}_{q}^{T} \overline{\mathbf{E}}_{q m}^{k}[\tau] \mathbf{B}_{m} \mathbf{d}_{m}[k-\tau] .
\end{aligned}
$$

The matrices $\left\{\overline{\mathbf{E}}_{q m}^{k}[k]\right\}$ are obtained by stacking column-wise the real and imaginary parts of the matrices $\left\{\mathbf{E}_{q m}^{k}[k]\right\}$, which are defined in (43). It has been shown in [86] that the degrees of freedom provided by $\left\{\overline{\mathbf{A}}_{q}, \mathbf{B}_{q}\right\}$ are insufficient to comply with the zero-interference constraint when $S=\min \left(N_{T}, N_{R}\right)$.
Owing to its close relation with BER and capacity, the minimization of the MSE becomes an appealing alternative. Denoting by $\mathbf{R}_{\eta_{q}}=\mathbb{E}\left\{\overline{\boldsymbol{\eta}}_{q}[k] \overline{\boldsymbol{\eta}}_{q}^{T}[k]\right\}$ the noise autocorrelation matrix, the MSE matrix on the $q$ th subcarrier is given by

$$
\begin{aligned}
\operatorname{MSE}_{q}= & \sum_{m, \tau} E_{S} \overline{\mathbf{A}}_{q}^{T} \overline{\mathbf{E}}_{q m}^{k}[\tau] \mathbf{B}_{m}\left(\overline{\mathbf{A}}_{q}^{T} \overline{\mathbf{E}}_{q m}^{k}[\tau] \mathbf{B}_{m}\right)^{T} \\
& +\overline{\mathbf{A}}_{q}^{T} \mathbf{R}_{\eta_{q}} \overline{\mathbf{A}}_{q}+E_{S} \mathbf{I}_{S}-2 E_{S} \overline{\mathbf{A}}_{q}^{T} \overline{\mathbf{E}}_{q q}^{k}[0] \mathbf{B}_{q} .
\end{aligned}
$$

Unfortunately, $\mathrm{MSE}_{q}$ is not jointly convex in $\overline{\mathbf{A}}_{q}$ and $\mathbf{B}_{q}$. As a consequence, we cannot resort to convex optimization theory to efficiently solve problems that depend on the MSE. Furthermore, we cannot optimize the MSE on each subcarrier independently, due to the partial overlapping between subcarriers. To alleviate the complexity, the authors in [87] propose to reformulate the exact MSE into a new expression that is easier to handle. In this regard, the MSE is replaced with this matrix

$$
\begin{aligned}
\mathrm{UB}_{q}= & \sum_{(m, \tau) \in \Omega_{q, k}} E_{S} b_{m} \overline{\mathbf{A}}_{q}^{T} \overline{\mathbf{E}}_{q m}^{k}[\tau]\left(\overline{\mathbf{A}}_{q}^{T} \overline{\mathbf{E}}_{q m}^{k}[\tau]\right)^{T} \\
& +\overline{\mathbf{A}}_{q}^{T} \mathbf{R}_{\eta_{q}} \overline{\mathbf{A}}_{q}+E_{S} \mathbf{I}_{S}-2 E_{S} \overline{\mathbf{A}}_{q}^{T} \overline{\mathbf{E}}_{q q}^{k}[0] \mathbf{B}_{q} \\
& +E_{S} \overline{\mathbf{A}}_{q}^{T} \overline{\mathbf{E}}_{q q}^{k}[0] \mathbf{B}_{q}\left(\overline{\mathbf{A}}_{q}^{T} \overline{\mathbf{E}}_{q q}^{k}[0] \mathbf{B}_{q}\right)^{T} .
\end{aligned}
$$

Given any vector $\mathbf{a} \in \mathbb{R}^{N_{T} \times 1}$, it can be verified that the entries in the diagonal of $\mathrm{MSE}_{q}$ are upper bounded by the diagonal elements of $\mathrm{UB}_{q}$, if

$$
\left\|\mathbf{a}^{T} \mathbf{B}_{m}\right\|_{2}^{2} \leq \lambda_{1}\left(\mathbf{B}_{m}^{T} \mathbf{B}_{m}\right)\|\mathbf{a}\|_{2}^{2} \leq b_{m}\|\mathbf{a}\|_{2}^{2} .
$$

The first inequality hinges on this well-known result $\operatorname{tr}(\mathbf{A B}) \leq$ $\operatorname{tr}(\mathbf{A}) \lambda_{1}(\mathbf{B})$, for symmetric matrix $\mathbf{A}$ and positive-semidefinite matrix $\mathbf{B}$ [88]. With the aim of further simplifying the notation the dominant eigenvalue of $\mathbf{B}_{m}^{T} \mathbf{B}_{m}$ is assumed to be upper bounded by $b_{m}$, which leads to the second inequality. Now, ICI and ISI terms, which correspond to the first line of $\mathrm{UB}_{q}$, depend on the constant $b_{m}$ and not on the precoder $\mathbf{B}_{m}$. The bound allows us to use the theory developed in [89] to find the MIMO precoding and decoding matrices. Bearing this in mind, it is proposed in [87] to minimize an arbitrary function $f_{0}\left(\left\{\left[\mathrm{UB}_{q}\right]_{l l}\right\}\right)$, which depends on the bound of the MSE. The optimization problem can be posed as follows:

$$
\begin{aligned}
& \underset{\left\{\overline{\mathbf{A}}_{q}, \mathbf{B}_{q}\right\}}{\operatorname{argmin}} f_{0}\left(\left[\left\{\mathrm{UB}_{q}\right]_{l l}\right\}\right) \\
& \text { s.t. } \sum_{q=0}^{M-1} \operatorname{tr}\left(\mathbf{B}_{q} \mathbf{B}_{q}^{T}\right) \leq P_{T} \\
& \lambda_{1}\left(\mathbf{B}_{m}^{T} \mathbf{B}_{m}\right) \leq b_{m}, \quad 0 \leq m \leq M-1,
\end{aligned}
$$

when there is a global power constraint given by $P_{T}$. Alternatively, individual power constraints can be used. The solution is known for several optimization problems, e.g. the sum-rate or the sum MSE, thanks to the unified framework developed in [89]. The additional constraints on the dominant eigenvalues imply that $\lambda_{l}\left(\mathbf{B}_{m}^{T} \mathbf{B}_{m}\right) \leq b_{m}$, for $1 \leq l \leq S$. The inequalities, which are required to facilitate analytical manipulations, do not 
affect the solvability of the problem and, thus, the structure of the transmit-receive processing is not altered (see [89]). It is worth mentioning that coefficients $\left\{b_{m}\right\}$ have to be judiciously selected, otherwise the bound may be too loose. The work in [86] discusses how to adjust the magnitude of $\left\{b_{m}\right\}$.

To perform closer to the optimum, the receive matrices can be updated, so that the exact MSE is minimized having fixed the transmit processing that optimizes $f_{0}\left(\left\{\left[\mathrm{UB}_{q}\right]_{l l}\right\}\right)$. Then, the optimized equalizers can be formulated with this closedform expression

$$
\overline{\mathbf{A}}_{q}=\left(\sum_{m, \tau} \overline{\mathbf{E}}_{q m}^{k}[\tau] \mathbf{B}_{m}\left(\overline{\mathbf{E}}_{q m}^{k}[\tau] \mathbf{B}_{m}\right)^{T}+\frac{\mathbf{R}_{\eta_{q}}}{E_{S}}\right)^{-1} \overline{\mathbf{E}}_{q q}^{k}[0] \mathbf{B}_{q}
$$

In multi-antenna configurations where $N_{R} \geq N_{T}=S$, the technique presented in this section gives satisfactory performance [87]. Otherwise, the good results cannot be guaranteed. For the simplest case of transmitting one stream, i.e. $S=1$, it is worth emphasizing that one can find objective functions other than the MSE, that govern the multi-tap processing design. The work developed in [90] shows that the signal to interference plus noise ratio (SINR) and the signal to leakage plus noise ratio (SLNR) are two performance metrics that allow FBMC/OQAM to remain competitive with OFDM, when multi-tap processing is applied at reception and transmission, respectively. Analogously to the strategy followed in this section, the original cost functions are relaxed and are replaced with lower bounds, which paves the way to obtaining closed-form solutions.

Without leaving single-user communications systems, let us now concentrate on the low-frequency selective case.

\section{B. Single-user communication systems. Low frequency selec- tive channels}

Under the assumption that the channel frequency response is almost flat at the subcarrier level, a new range of possibilities can be considered for designing the transceiver. Bearing this in mind and borrowing the notation from Section II-B, it is assumed that the $S$ symbols to be spatially multiplexed are linearly mapped onto the $N_{T}$ antennas as follows: $\mathbf{v}_{m}[k]=$ $\theta_{m}[k] \mathbf{B}_{m} \mathbf{d}_{m}[k]$. The matrix $\mathbf{B}_{m} \in \mathbb{C}^{N_{T} \times S}$ is in charge of precoding the symbols on the $m$ th subcarrier. At the receive side, the $q$ th output of the AFB is linearly transformed through the matrix $\mathbf{A}_{q} \in \mathbb{C}^{N_{R} \times S}$, resulting in $\mathbf{z}_{q}[k]=\mathbf{A}_{q}^{H} \mathbf{y}_{q}[k]$. Finally, the transmitted data is estimated after compensating the phase term and extracting the real part, i.e. $\check{\mathbf{d}}_{q}[k]=\Re\left(\theta_{q}^{*}[k] \mathbf{z}_{q}[k]\right)$. If it is sought to implement the singular value decomposition beamforming intended for OFDM, it is essential to make sure that the model 2) in (26) accurately characterizes the system model. Moreover, when the $q$ th subcarrier is analyzed, it is assumed that the channel seen by the desired signal as well as the interfering signals is the same and equal to $\mathbf{H}_{q}$. Then, the global communication system becomes

$$
\begin{aligned}
\check{\mathbf{d}}_{q}[k]= & \Re\left(\mathbf{A}_{q}^{H} \mathbf{H}_{q} \mathbf{B}_{q}\right) \mathbf{d}_{q}[k]+\Re\left(\theta_{q}^{*}[k] \mathbf{A}_{q}^{H} \mathbf{w}_{q}[k]\right)+ \\
& \sum_{(m, \tau) \in \Omega_{q, k}} \Re\left(\Gamma_{q m}^{k}[\tau] \mathbf{A}_{q}^{H} \mathbf{H}_{q} \mathbf{B}_{m}\right) \mathbf{d}_{m}[k-\tau] .
\end{aligned}
$$

Based on this formulation, the optimal solution in the MSE sense for OFDM systems [89] is given by

$$
\begin{gathered}
\mathbf{A}_{q}=\left(\mathbf{H}_{q} \mathbf{B}_{q} \mathbf{B}_{q}^{H} \mathbf{H}_{q}^{H}+\mathbf{R}_{w_{q}}\right)^{-1} \mathbf{H}_{q} \mathbf{B}_{q} \\
\mathbf{R}_{w_{q}}=\mathbb{E}\left\{\mathbf{w}_{q}[k] \mathbf{w}_{q}^{H}[k]\right\}
\end{gathered}
$$

and $\mathbf{B}_{q}=\mathbf{V}_{q}$, where $\mathbf{V}_{q} \in \mathbb{C}^{N_{T} \times S}$ has as columns the $S$ dominant eigenvectors of this matrix $\mathbf{H}_{q}^{H} \mathbf{R}_{w_{q}}^{-1} \mathbf{H}_{q}$. The equivalent channel seen by the stream $\mathbf{d}_{m}[k-\tau]$ reads $\mathbf{A}_{q}^{H} \mathbf{H}_{q} \mathbf{B}_{m}=$ $\left(\mathbf{I}_{s}+\boldsymbol{\Lambda}_{q}\right)^{-1} \boldsymbol{\Lambda}_{q} \mathbf{V}_{q}^{H} \mathbf{V}_{m}$, where $\boldsymbol{\Lambda}_{q} \in \mathbb{R}^{S \times S}$ denotes the diagonal matrix that is constituted by the real-valued eigenvalues of $\mathbf{H}_{q}^{H} \mathbf{R}_{w_{q}}^{-1} \mathbf{H}_{q}$. Presuming that the channel is constant in three consecutive subcarriers, i.e. $\mathbf{H}_{q-1}=\mathbf{H}_{q}=\mathbf{H}_{q+1}$, the equivalent channel $\mathbf{A}_{q}^{H} \mathbf{H}_{q} \mathbf{B}_{m}$ becomes a diagonal real-valued matrix. In this situation, the decision variables are expressed as

$$
\begin{array}{r}
\check{\mathbf{d}}_{q}[k]= \\
\quad \sum_{(m, \tau) \in \Omega_{q, k}} \Re\left(\Gamma_{q m}^{k}[\tau]\right)\left(\mathbf{I}_{s}+\boldsymbol{\Lambda}_{q}\right)^{-1} \boldsymbol{\Lambda}_{q} \mathbf{d}_{q}[k]+\Re\left(\theta_{q}^{*}[k] \mathbf{A}_{q}^{H} \mathbf{w}_{q}[k]\right)+ \\
\mathbf{d}_{m}[k-\tau] .
\end{array}
$$

If the pulses satisfy (10), then the orthogonality is preserved as long as $\mathbf{H}_{q-1}=\mathbf{H}_{q}=\mathbf{H}_{q+1}$. Otherwise, the MIMO processing tailored to OFDM cannot be directly applied to FBMC/OQAM systems without incurring in ISI and ICI.

Assuming that $\mathbf{H}_{q-1} \neq \mathbf{H}_{q} \neq \mathbf{H}_{q+1}$, it will be shown that the resilience against the channel frequency selectivity can be increased. Provided that the pulses comply with (10), the system model can be compactly formulated as it is proposed in [91] with this matrix notation

$$
\begin{aligned}
\check{\mathbf{d}}_{q}[k]= & \overline{\mathbf{A}}_{q}^{T} \check{\mathbf{H}}_{q} \overline{\mathbf{B}}_{q} \mathbf{d}_{q}[k]+\overline{\mathbf{A}}_{q}^{T} \overline{\boldsymbol{\eta}}_{q}[k] \\
& -\sum_{m=q-1}^{q+1} \overline{\mathbf{A}}_{q}^{T} \hat{\mathbf{H}}_{m} \overline{\mathbf{B}}_{m} \Im\left(\mathbf{i}_{q m}[k]\right) .
\end{aligned}
$$

The real-valued representation of $\check{\mathbf{d}}_{q}[k]$ relies on the notation introduced in Section I, the definition of $\boldsymbol{\eta}_{q}=\theta_{q}^{*} \mathbf{w}_{q}$, the augmented channel matrices

$$
\begin{aligned}
& \check{\mathbf{H}}_{m}=\left[\begin{array}{cc}
\Re\left(\mathbf{H}_{m}\right) & -\Im\left(\mathbf{H}_{m}\right) \\
\Im\left(\mathbf{H}_{m}\right) & \Re\left(\mathbf{H}_{m}\right)
\end{array}\right] \\
& \hat{\mathbf{H}}_{m}=\left[\begin{array}{cc}
\Im\left(\mathbf{H}_{m}\right) & \Re\left(\mathbf{H}_{m}\right) \\
-\Re\left(\mathbf{H}_{m}\right) & \Im\left(\mathbf{H}_{m}\right)
\end{array}\right]
\end{aligned}
$$

and the pure imaginary vector $\mathbf{i}_{q m}[k]$, namely

$$
\mathbf{i}_{q m}[k]=\sum_{\tau=-L_{g_{1}}}^{L_{g_{2}}} \Gamma_{q m}^{k}[\tau] \mathbf{d}_{m}[k-\tau], \quad m \neq q
$$




$$
\mathbf{i}_{q q}[k]=\sum_{\substack{\tau=-L_{g_{1}} \\ \tau \neq 0}}^{L_{g_{2}}} \Gamma_{q q}^{k}[\tau] \mathbf{d}_{q}[k-\tau] .
$$

The complexity burden can be reduced at the receive side if equalizers are constrained to only have real-valued components. Then, $\overline{\mathbf{A}}_{q} \in \mathbb{R}^{N_{R} \times S}$ and the real-valued channel matrices become

$$
\begin{aligned}
\check{\mathbf{H}}_{m} & =\left[\begin{array}{ll}
\Re\left(\mathbf{H}_{m}\right) & -\Im\left(\mathbf{H}_{m}\right)
\end{array}\right] \\
\hat{\mathbf{H}}_{m} & =\left[\begin{array}{ll}
\Im\left(\mathbf{H}_{m}\right) & \Re\left(\mathbf{H}_{m}\right)
\end{array}\right] .
\end{aligned}
$$

As it is anticipated in subsection II-D, the consequence of representing the system model with real variables is that real and imaginary parts are independently processed, which means that $\overline{\mathbf{B}}_{q}$ and $\overline{\mathbf{A}}_{q}$ perform a WL processing at transmission and reception, respectively [56]. Building upon the realvalued system model, two designs are derived to enhance the robustness against the modulation-induced interference, when compared to the strategy of implementing the linear processing described in [89], as it is proposed in [41], [92]. Unlike the technique proposed in Section IV-A2, the strategy followed in low-frequency selective channels consists in using real- and complex-valued coefficients to build equalizers and precoders, respectively. With this configuration, it will be demonstrated that the number of streams supported by the system is $S \leq \min \left(N_{T}, N_{R}\right)$.

1) Zero forcing method: Adopting the notation of (81), the authors in [93] realized that ISI and ICI terms are canceled if precoders are designed to satisfy $\hat{\mathbf{H}}_{q} \overline{\mathbf{B}}_{q}=\mathbf{0}$. Following the same philosophy as [94], the ZF approach relies on projecting the MIMO precoding matrices onto the null space of the interference matrix, i.e. $\hat{\mathbf{H}}_{q}$. Nevertheless, this strategy only makes sense when equalizers are real-valued. It can be checked that in the most general case where the coefficients of $\overline{\mathbf{B}}_{q}$ and $\overline{\mathbf{A}}_{q}$ are complex-valued, the matrices (82) and (83) span the same subspace. As a consequence, there are not enough degrees of freedom to remove the interference without eliminating the desired signal. Conversely, matrices (86) and (87) span different subspaces. Hence, if $\Im\left(\mathbf{A}_{q}\right)=\mathbf{0}$, it is possible to find a matrix $\mathbf{V}_{q} \in \mathbb{R}^{2 N_{T} \times 2 N_{T}-N_{R}}$ that complies with $\hat{\mathbf{H}}_{q} \mathbf{V}_{q}=\mathbf{0}$ and $\check{\mathbf{H}}_{q} \mathbf{V}_{q} \neq \mathbf{0}$. Interference cancellation can be achieved by selecting the vectors that span the null space of $\hat{\mathbf{H}}_{q}$ as the columns of $\mathbf{V}_{q}$. Without loss of generality the MIMO precoding matrix can be factorized as the product of the inner precoder $\overline{\mathbf{B}}_{q}^{i}$ and the outer precoder $\overline{\mathbf{B}}_{q}^{o}$ as follows: $\overline{\mathbf{B}}=\overline{\mathbf{B}}_{q}^{i} \overline{\mathbf{B}}_{q}^{o}$. Using $\mathbf{V}_{q}$ as the inner precoder, the interference that leaks from the subcarriers $\{q-1, q, q+1\}$ is removed, yielding

$$
\check{\mathbf{d}}_{q}[k]=\overline{\mathbf{A}}_{q}^{T} \check{\mathbf{H}}_{q} \mathbf{V}_{q} \overline{\mathbf{B}}_{q}^{o} \mathbf{d}_{q}[k]+\overline{\mathbf{A}}_{q}^{T} \overline{\boldsymbol{\eta}}_{q}[k] .
$$

With the ZF solution we end up with a $N_{R} \times 2 N_{T}-N_{R}$ MIMO communication system that is free of interference. Therefore, it is possible to benefit from the theory developed in the OFDM context just realizing that the channel, which is given by $\check{\mathbf{H}}_{q} \mathbf{V}_{q}$, has embedded the inner precoder. Depending on the performance metric to be optimized, the rest of the degrees

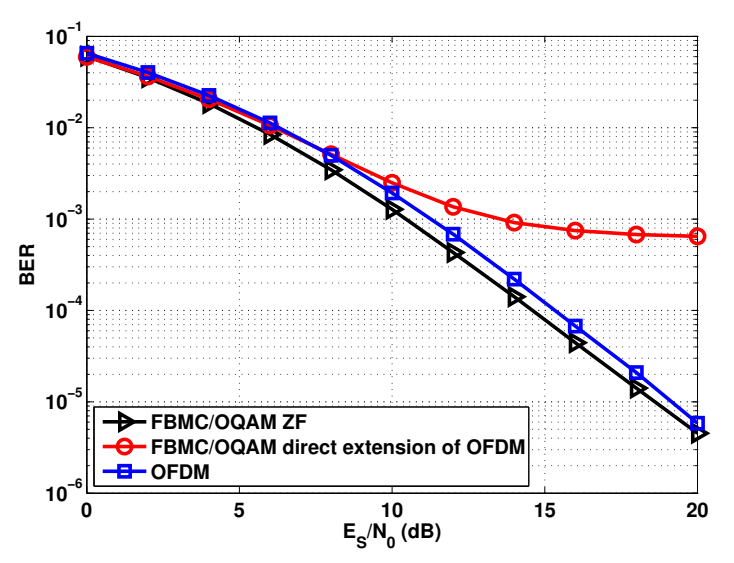

Figure 15. Comparison of the BER performances of different schemes in a single-user MIMO system where $N_{T}=4, N_{R}=2, S=2$.

of freedom, i.e. the matrix pairs $\left\{\overline{\mathbf{A}}_{q}, \overline{\mathbf{B}}_{q}^{o}\right\}$, can be jointly designed as the authors propose in [89]. Due to the processing carried out by the inner precoder, the spatial channel gains are less spread out with respect to the case where no projection is needed [93]. As a result, satisfactory performance is not guaranteed unless all the modes are active, i.e. $S=N_{R} \leq N_{T}$.

2) Coordinated beamforming: An iterative scheme has been proposed in [95] to design beamformers when $S \leq$ $N_{T} \leq N_{R}$ and $S<2 N_{T}$ are simultaneously satisfied. This technique alleviates the dimensionality constraint on the $\mathrm{ZF}$ method, i.e., its performance is only satisfactory when the constraints $S=N_{R} \leq N_{T}$ hold. The enhancement lies in establishing a dependency between precoders and equalizers. When it comes to designing precoders, this translates into coupling the channel to the decoding matrix, so that the equivalent channel matrices in (81) read $\overline{\mathbf{A}}_{q}^{T} \hat{\mathbf{H}}_{q}$ and $\overline{\mathbf{A}}_{q}^{T} \check{\mathbf{H}}_{q}$. To illustrate how the receive processing impacts the precoding design and vice versa, let us focus on the $p$ th iteration, where the MIMO decoding and precoding matrices are denoted by $\overline{\mathbf{A}}_{q}^{(p)} \in \mathbb{R}^{N_{R} \times S}$ and $\overline{\mathbf{B}}_{q}^{(p)} \mathbb{C}^{N_{T} \times S}$, respectively. After executing the $(p-1)$ th iteration, the desired signal becomes

$$
\begin{aligned}
\check{\mathbf{d}}_{q}[k]= & \overline{\mathbf{A}}_{q}^{(p-1)^{T}} \check{\mathbf{H}}_{q} \overline{\mathbf{B}}_{q}^{(p-1)} \mathbf{d}_{q}[k]+\overline{\mathbf{A}}_{q}^{(p-1)^{T}} \overline{\boldsymbol{\eta}}_{q}[k] \\
& -\sum_{m=q-1}^{q+1} \overline{\mathbf{A}}_{q}^{(p-1)^{T}} \hat{\mathbf{H}}_{m} \overline{\mathbf{B}}_{m}^{(p-1)} \Im\left(\mathbf{i}_{q m}[k]\right) .
\end{aligned}
$$

At the $p$ th iteration, first, the ZF method is applied on the equivalent channel matrices $\hat{\mathbf{H}}_{e_{q}}^{(p)}=\overline{\mathbf{A}}_{q}^{(p-1)^{T}} \hat{\mathbf{H}}_{q} \in$ $\mathbb{R}^{S \times 2 N_{T}}$ and $\check{\mathbf{H}}_{e_{q}}^{(p)}=\overline{\mathbf{A}}_{q}^{(p-1)^{T}} \check{\mathbf{H}}_{q} \in \mathbb{R}^{S \times 2 N_{T}}$. Consequently, the precoding matrix in the $p$ th iteration is factorized as $\overline{\mathbf{B}}_{q}^{(p)}=\overline{\mathbf{B}}_{q}^{i(p)} \overline{\mathbf{B}}_{q}^{o(p)}$. The inner precoder is obtained such that $\hat{\mathbf{H}}_{e_{q}}^{(p)} \overline{\mathbf{B}}_{q}^{i(p)}=\mathbf{0}$. Since the equivalent channel embraces the receive processing, the number of columns of $\overline{\mathbf{B}}_{q}^{i(p)}$ is $2 N_{T}-S$. Then, the outer precoder has as columns the $S$ right singular vectors of $\check{\mathbf{H}}_{e_{q}}^{(p)} \overline{\mathbf{B}}_{q}^{i(p)} \in \mathbb{R}^{S \times 2 N_{T}-S}$, which are associated to the dominant singular values. Finally, considering a certain criterion for the receive processing at the receiver, e.g., MMSE, or $\mathrm{ZF}, \overline{\mathbf{A}}_{q}^{(p)}$ is computed based on the equivalent channel that 


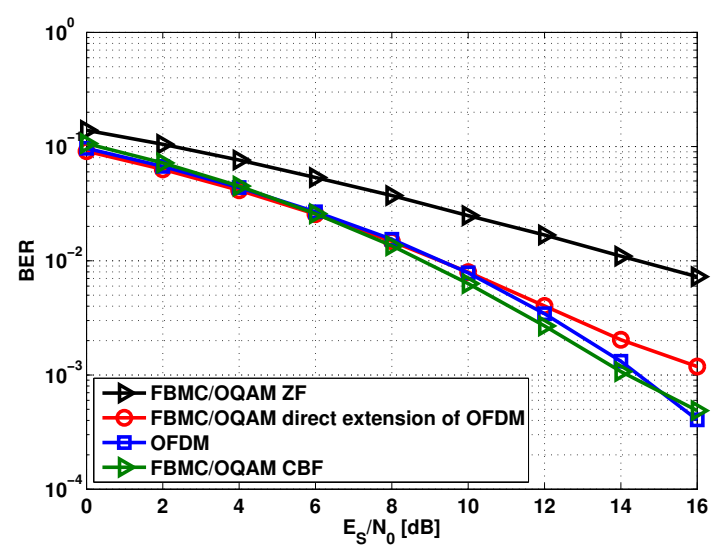

Figure 16. Comparison of the BER performances of different schemes in a single-user MIMO system where $N_{T}=6, N_{R}=6, S=5$.

takes the form $\check{\mathbf{H}}_{q} \overline{\mathbf{B}}_{q}^{(p)}=\Re\left(\mathbf{H}_{q} \mathbf{B}_{q}^{(p)}\right) \in \mathbb{R}^{N_{R} \times S}$.

The performance of the $\mathrm{ZF}$ method depends on the eigenvalues of $\check{\mathbf{H}}_{q} \overline{\mathbf{B}}_{q}^{i}$. The beauty of the coordinated beamforming is that the performance is determined by the eigenvalues of $\check{\mathbf{H}}_{e_{q}}^{(p)} \overline{\mathbf{B}}_{q}^{i(p)}$, which mainly depend on the channel and the receive processing. Hence, it can be understood that the iterative procedure seeks for the equivalent channels $\hat{\mathbf{H}}_{e_{q}}^{(p)}$ and $\check{\mathbf{H}}_{e_{q}}^{(p)}$, which maximize the performance achieved by the ZF method.

3) Performance validation: We consider these two MIMO systems: $N_{T}=4, N_{R}=2, S=2$ and $N_{T}=N_{R}=6, S=5$. The number of subcarriers is $M=1024$ and the symbols belong to the 16-QAM constellation. The system parameters correspond to the scenario 2, which is detailed in Table II. Equal power allocation is assumed on all data streams and subcarriers. In Fig. 15 and Fig. 16, the BER performances of three schemes for FBMC/OQAM systems are presented and compared to that of an OFDM system. The beamformers in OFDM are designed to achieve a diagonal structure on each subcarrier [89].

The transmission scheme detailed at the beginning of Section IV-B is a straightforward extension of the OFDM case and relies on the assumption that the channel frequency responses remain the same across adjacent subcarriers. As the EVA channel exhibits frequency selectivity and such an assumption is therefore violated, the performance of this scheme degrades severely especially in the low noise regime. Eliminating the interference via $\mathrm{ZF}$ precoding, the error floor is completely removed. The degradation suffered when ZF is applied is non-existing in Fig. 15. Nonetheless, as anticipated in Section IV-B2 if $S=N_{R} \leq N_{T}$ is not satisfied, then the ZF performs poorly. In those multi-antenna configurations where the ZF does not achieve satisfactory performance, the coordinated beamforming (CBF) appears as a good candidate to improve the performance. Note that for the CBF technique [95] the complete elimination of the interference requires that $\overline{\mathbf{A}}_{q}=\overline{\mathbf{A}}_{m}(m=q-1, q+1)$ [96]. Since the EVA channel is used, this condition is not fulfilled. Consequently, there still exists residual intrinsic interference, and in the low noise regime the performance of the $\mathrm{CBF}$ scheme is slightly worse compared to the case of OFDM systems.

\section{Multi-user communication systems for broadcast channels}

The amount of publications devoted to combining FBMC/OQAM with SDMA in a multi-user context is quite limited. The communication system that is studied in this section consists in a transmitter serving $N_{U}$ decentralized users in the same time/frequency resources. In highly frequency selective channels, the transmit processing is based on performing multi-tap filtering to separate users [97]-[99]. Then, the equalizer can be separately designed from the precoder to remove the residual interference. The adoption of this suboptimal strategy highlights the fact that the literature addressing multi-user communication systems for highly frequency selective channels is not very extensive. In consequence, the rest of the section will be devoted to studying the solutions intended for low frequency selective channels, which are more mature.

Along this section these definitions will be used: $N_{R}=$ $\sum_{l} N_{R_{l}}$ and $S=\sum_{l} S_{l}$. The variables $N_{R_{l}}$ and $S_{l}$ denote the number of receive antennas and streams, respectively, associated to the $l$ th user. Let $\mathbf{d}_{l q}[k] \in \mathbb{R}^{S_{l} \times 1}$ denote the data intended to the $l$ th user, which is transmitted on the $q$ th subcarrier and the $k$ th time instant. The noise vector that contaminates the reception of the $l$ th user at the output of the AFB is denoted by $\mathbf{w}_{l q}[k] \in \mathbb{C}^{N_{R_{l}} \times 1}$.

In low frequency selective channels, the overall transceiver equation is written as

$$
\begin{aligned}
\check{\mathbf{d}}_{l q}[k] & =\sum_{u=1}^{N_{U}} \Re\left(\mathbf{A}_{l q}^{H} \mathbf{H}_{l q} \mathbf{B}_{u q}\right) \mathbf{d}_{u q}[k]+\Re\left(\theta_{q}^{*}[k] \mathbf{A}_{l q}^{H} \mathbf{w}_{l q}[k]\right) \\
& +\sum_{u=1}^{N_{U}} \sum_{(m, \tau) \in \Omega_{q, k}} \Re\left(\Gamma_{q m}^{k}[\tau] \mathbf{A}_{l q}^{H} \mathbf{H}_{l m} \mathbf{B}_{u m}\right) \mathbf{d}_{u m}[k-\tau],
\end{aligned}
$$

for $1 \leq l \leq N_{U}$. The triplet $\left\{\mathbf{A}_{l q}, \mathbf{H}_{l q}, \mathbf{B}_{l q}\right\}$ accounts for the equalizer, the channel and the precoder associated to the $q$ th subcarrier and the $l$ th user. Bearing in mind the global communication system written in (90), two approaches are presented in the following to achieve SDMA in FBMC/OQAM systems, namely the block diagonalization (BD) concept and the spatial Tomlinson-Harashima precoder (STHP).

1) Block diagonalization: SDMA via BD was first proposed in [94]. The idea is to achieve interference-free data multiplexing so that multiple users are served in the downlink, while ensuring that the signal intended for a given user does not interfere the reception of the unintended users. This subsection shows that the technique presented in [94] can be implemented on a per-subcarrier basis in the FBMC/OQAM context [100]. The first step consists in removing inter-user interference (IUI). To this end, the interference matrix $\tilde{\mathbf{H}}_{l q}$ is defined for $1 \leq l \leq N_{U}$, which is obtained by stacking column-wise the channel matrices of all users except that of the $l$ th one, that is

$$
\tilde{\mathbf{H}}_{l q}=\left[\mathbf{H}_{1 q}^{T} \cdots \mathbf{H}_{l-1 q}^{T} \mathbf{H}_{l+1 q}^{T} \cdots \mathbf{H}_{N_{U} q}^{T}\right]^{T} .
$$

Based on this definition, it is guaranteed that leakage is removed if $\tilde{\mathbf{H}}_{l q} \mathbf{B}_{l q}=\mathbf{0}$. To this end, precoders are decomposed 


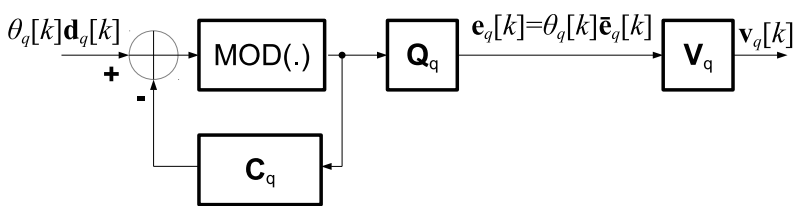

Figure 17. Block diagram of the STHP adapted to the FBMC/OQAM modulation scheme.

as $\mathbf{B}_{l q}=\mathbf{U}_{l q} \mathbf{F}_{l q}$, where $\mathbf{U}_{l q} \in \mathbb{C}^{N_{T} \times N_{T}-\sum_{u \neq l} N_{R_{u}}}$ spans the null space of $\mathbf{H}_{l q}$. It is important to recall that the effect of IUI is completely removed, as long as $\sum_{u \neq l} N_{R_{u}}<N_{T}, \forall l$. Then, (90) can be recast as

$$
\begin{aligned}
\check{\mathbf{d}}_{l q}[k] & =\Re\left(\mathbf{A}_{l q}^{H} \mathbf{H}_{l q} \mathbf{U}_{l q} \mathbf{F}_{l q}\right) \mathbf{d}_{l q}[k]+\Re\left(\theta_{q}^{*}[k] \mathbf{A}_{l q}^{H} \mathbf{w}_{l q}[k]\right) \\
& +\sum_{(m, \tau) \in \Omega_{q, k}} \Re\left(\Gamma_{q m}^{k}[\tau] \mathbf{A}_{l q}^{H} \mathbf{H}_{l m} \mathbf{U}_{l m} \mathbf{F}_{l m}\right) \mathbf{d}_{l m}[k-\tau] .
\end{aligned}
$$

Now one can deal with ISI and ICI by designing $\mathbf{A}_{l q} \in$ $\mathbb{R}^{N_{R_{l}} \times S_{l}}$ and $\mathbf{F}_{l q} \in \mathbb{C}^{N_{T}-\sum_{u \neq l} N_{R_{u}} \times S_{l}}$ according to the derivation steps detailed in either subsection IV-B1 or subsection IV-B2. Therefore, when selecting the number of transmittable streams, we should reckon with the dimensionality constraints. The conclusion is that $\mathrm{BD}$ and $\mathrm{ZF}$ can be successfully combined if the number of streams and antennas are chosen in accordance with this expression $S_{l}=N_{R_{l}} \leq$ $N_{T}-\sum_{u \neq l} N_{R_{u}}$. By contrast, the successful application of $\mathrm{CBF}$ in conjunction with $\mathrm{BD}$, which is referred to as intrinsic interference mitigating coordinate beamforming (IIM-CBF) [96], requires the fulfillment of $S_{l} \leq N_{T}-\sum_{u \neq l} N_{R_{u}} \leq N_{R_{l}}$ and $S_{l} \leq 2 N_{T}-\sum_{u \neq l} 2 N_{R_{u}}$.

It is worth noting that in case $N_{T}<N_{R}$, the null space of (91) is empty. Thus, the BD technique [94] cannot be employed. An iterative solution to such scenarios was provided in [96], called IIM-CBF 2.

2) Spatial Tomlinson-Harashima Precoder: As an alternative to the BD technique, the spatial Tomlinson Harashima precoder (STHP) can be applied to achieve SDMA [101], [102]. Concentrating on the FBMC/OQAM modulation scheme, it can be proven that the STHP is not able to cope with the modulation-induced interference unless the channel frequency response is flat in three consecutive subcarriers [103], rendering the original solution impractical. The degradation is aggravated if the fact that the symbol alphabet is constituted by real-valued elements is ignored. To remedy this problem and provide a higher degree of robustness against the channel frequency selectivity, the symbols are pre-processed as Fig. 17 shows [103], [104]. The proposed nonlinear processing is conceived similarly to the classical STHP. However, due to the characteristics of FBMC/OQAM, some modifications have been introduced. The main novelty comes from the concatenation of precoders $\mathbf{V}_{q} \in \mathbb{C}^{N_{T} \times 2 N_{T}-N_{R}}$ and $\mathbf{Q}_{q} \in \mathbb{R}^{2 N_{T}-N_{R} \times S}$. Building upon the work carried out in subsection IV-B1, the target of the inner precoder is to project the desired and the unwanted signals onto orthogonal subspaces to facilitate the removal of the interference at the

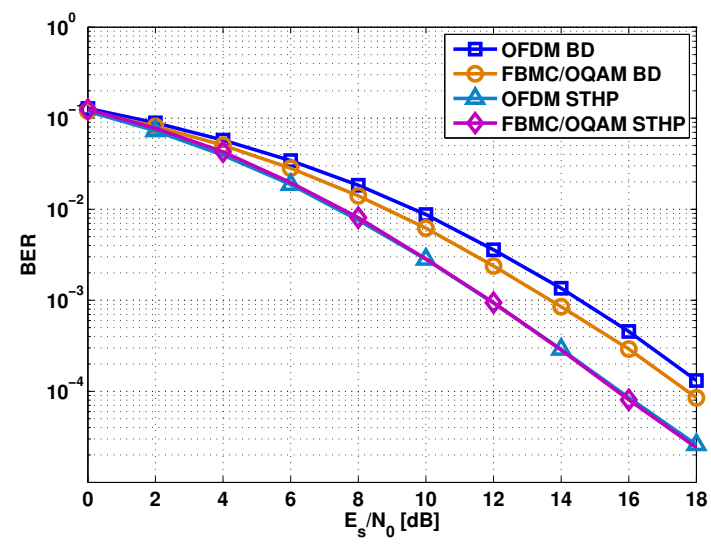

Figure 18. Comparison of the BER performances of different schemes in a multi-user MIMO downlink system where $N_{U}=3, N_{T}=8, N_{R}=6$, $S=6$, and the EVA channel model is considered.

receive side. To this end, it is necessary to define the matrix $\mathbf{H}_{m}=\left[\mathbf{H}_{1 m}^{T} \cdots \mathbf{H}_{N_{U} m}^{T}\right]^{T} \in \mathbb{C}^{N_{R} \times N_{T}}$. Imposing $2 N_{T} \geq N_{R}$, we can find a matrix $\mathbf{V}_{q} \in \mathbb{C}^{N_{T} \times 2 N_{T}-N_{R}}$ that guarantees $\Im\left(\mathbf{H}_{q} \mathbf{V}_{q}\right)=\mathbf{0}$. Then, the intrinsic interference is eliminated after taking the real part of the received signal if three conditions are satisfied. The first one consists in restricting the MIMO decoding matrices to be real-valued, i.e. $\mathbf{A}_{l q} \in$ $\mathbb{R}^{N_{R_{l}} \times S_{l}}$. The second condition states that the phases of the symbols have to be characterized by (13), so that depending on the FT position symbols are either real or pure imaginary. The third requirement stipulates that the PR property given by (10) is fulfilled. Under these three assumptions, the vector $\check{\mathbf{d}}_{q}[k]=\left[\check{\mathbf{d}}_{1 q}[k] \cdots \check{\mathbf{d}}_{N_{U} q}[k]\right]^{T}$ is expressed as

$$
\check{\mathbf{d}}_{q}[k]=\mathbf{A}_{q}^{T} \Re\left(\mathbf{H}_{q} \mathbf{V}_{q}\right) \overline{\mathbf{e}}_{q}[k]+\Re\left(\theta_{q}^{*}[k] \mathbf{A}_{q}^{T} \mathbf{w}_{q}[k]\right),
$$

where $\mathbf{w}_{q}[k]=\left[\mathbf{w}_{1 q}^{T}[k] \cdots \mathbf{w}_{N_{U} q}^{T}[k]\right]^{T} \in \mathbb{C}^{N_{R} \times 1}$. The vector $\overline{\mathbf{e}}_{q}[k] \in \mathbb{R}^{S \times 1}$ is the result of processing $\mathbf{d}_{q}[k]=$ $\left[\mathbf{d}_{1 q}^{T}[k] \cdots \mathbf{d}_{N_{U} q}^{T}[k]\right]^{T} \in \mathbb{R}^{S \times 1}$, with the STHP. As Fig. 17 shows, the nonlinear part of the precoder preserves the phase of the input symbols, which is decisive to end up with (93). Accepting (93) as the true expression, it follows that the global decoding matrix is restricted to be block diagonal, because receivers are not allowed to cooperate. Hence, $\mathbf{A}_{q}$ is constructed as follows:

$$
\mathbf{A}_{q}=\left[\begin{array}{ccc}
\mathbf{A}_{1 q} & \cdots & \mathbf{0} \\
& \ddots & \\
\mathbf{0} & \cdots & \mathbf{A}_{N_{U} q}
\end{array}\right] .
$$

Now it becomes evident that the rest of parameters, i.e. $\mathbf{C}_{q} \in$ $\mathbb{R}^{N_{R} \times N_{R}}, \mathbf{Q}_{q} \in \mathbb{R}^{2 N_{T}-N_{R} \times N_{R}}$ and $\mathbf{A}_{q} \in \mathbb{R}^{N_{R} \times S}$, can be designed according to the classical STHP, by performing the QR-decomposition of the matrix $\Re\left(\mathbf{H}_{q} \mathbf{V}_{q}\right)$. It is pertinent to stress that the proposed STHP imposes a diagonal structure on $\mathbf{A}_{q}$, as well as this dimensionality constraint $2 N_{T}-N_{R} \geq$ $N_{R}=S$. To adapt the STHP to the case where $N_{T}<N_{R}$, an iterative scheme, which is inspired by the CBF addressed in subsection IV-B2, is proposed in [104]. 


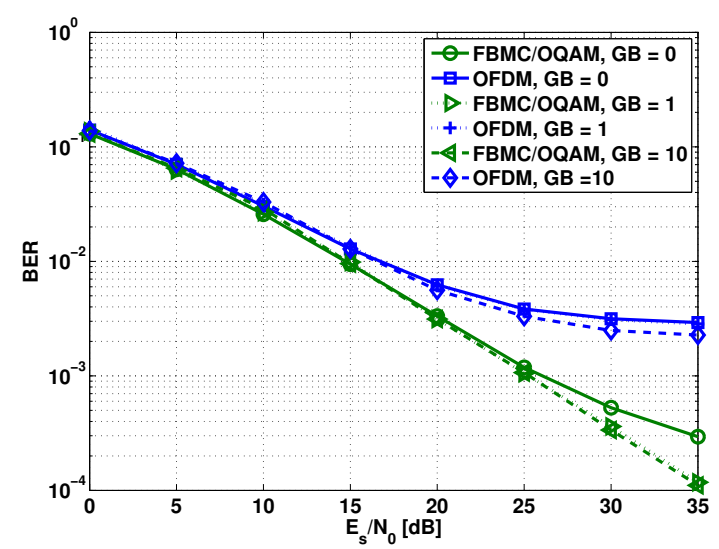

Figure 19. Comparison between FBMC/OQAM and OFDM for a two-user uplink scenario in the presence of symbol timing offsets in the range of $(T / 8$, $T / 4$ ), where the two users and the base station are each equipped with two antennas, and the EPA channel model is considered (GB - guard band, in terms of the number of subcarriers).

It is essential to highlight that the projection performed by $\mathbf{V}_{q}$ has a negative impact on the proposed STHP, when compared to systems that can manage without projecting the transmitted signals.

3) Performance validation: A multi-user MIMO downlink setting is considered where $N_{U}=3, N_{T}=8$, and $N_{R}=S=$ 6 . The number of subcarriers is $M=1024$ and the symbols belong to the 16-QAM constellation. The system parameters are set according to the scenario 2 as detailed in Table II. In addition, we assume equal power allocation on all data streams and subcarriers. A BER performance comparison of two schemes for FBMC/OQAM systems and their counterparts for OFDM systems is presented in Fig. 18.

It can be observed that FBMC/OQAM systems where the BD scheme [100] is employed achieves a slightly better performance compared to OFDM systems with the BD technique [94]. The gain results from the fact that the CP is not needed in FBMC/OQAM systems. On the other hand, the non-linear precoding scheme, the STHP for FBMC/OQAM systems [103], [104], outperforms the BD approach that belongs to the category of linear precoding. We can also see that the performance of FBMC/OQAM and OFDM systems with the STHP is almost the same. The negligible gain reveals that extending a non-linear scheme to fit in FBMC/OQAM-based systems leads to a slightly different result from the case of linear precoding schemes.

4) Performance validation of FBMC/OQAM for the asynchronous multi-user transmission: The greater robustness of FBMC/OQAM against synchronization errors compared to OFDM has been extensively verified in the literature. Furthermore, an exhaustive study has recently been presented in [58], where the multi-user distortion is characterized. Consider a multi-user MIMO uplink system where the user terminals are each assigned a group of consecutive subcarriers and transmit to the base station at the same time [105]. At the receive side, it is assumed that users can be perfectly separated and, therefore, transmit and receive signal processing techniques for point-to-point systems are employed. The first case analyzed

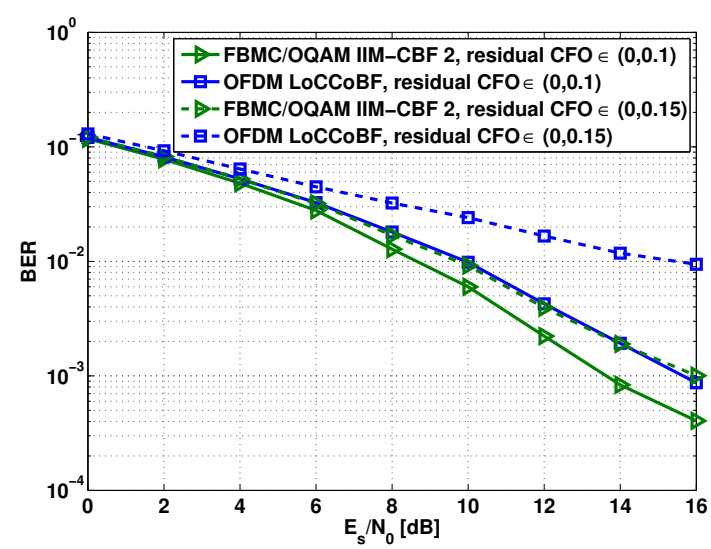

Figure 20. Comparison of the BER performances of different schemes in a multi-user MIMO downlink system in the presence of residual CFO (normalized by the subcarrier spacing) where $N_{U}=4, N_{T}=8, N_{T}=12$, $S=8$, and the EPA channel model is considered.

in this section focuses on the spatial multiplexing transmission without precoding. The single-tap MMSE receiver described in Section III-B1 has been implemented to separate the streams of each user. Due to the fact that it is difficult to guarantee that the signals from different users arrive at the base station simultaneously, such a system is very prone to misalignments in the time domain. On the other hand, the loss of synchronization in the frequency domain contaminates the orthogonality between subcarriers. Taking a two-user setting as an example, Figure 19 shows that in the presence of symbol timing offsets uniformly distributed in the range $(T / 8, T / 4)$, where $T$ represents the symbol period. The number of subcarriers is $M=1024$ and the symbols are drawn from the 4-QAM constellation. The BER curves show that a single subcarrier as the guard band suffices for FBMC/OQAM to achieve the separation of signals from different users. This was also verified in [106] in the context of distributed MIMO-FBMC/OQAM systems. By contrast, in case of OFDM, even if a much larger guard band with ten subcarriers is employed at the price of a severe loss of the spectral efficiency, an error floor in the BER curve is observed. This has been analytically confirmed in the study [58]. Moreover, the impact of residual carrier frequency offset (CFO) is investigated in [105], showing that FBMC/OQAM significantly outperforms OFDM in the presence of frequency misalignments. FBMC/OQAM is also much less sensitive to the increase of the residual CFO compared to OFDM. As the maximum residual $\mathrm{CFO}$ is increased from 0.1 subcarrier spacing to 0.15 , the performance degradation of FBMC/OQAM is much smaller than that of OFDM. FBMC/OQAM is, therefore, a promising multicarrier modulation scheme for the multi-user MIMO uplink.

In addition, a similar performance evaluation of FBMC/OQAM-based multi-user MIMO downlink systems with SDMA that suffer from residual CFO has been conducted in [96]. The IIM-CBF 2 scheme (mentioned in Section IV-C1) is employed. As shown in Figure 20, a four-user scenario is considered where the aggregated number of receive antennas of the user terminals exceeds the number of transmit antennas 
at the base station, and the residual CFOs (normalized by the subcarrier spacing) range either from 0 to 0.1 or from 0 to 0.15 . The number of subcarriers is $M=1024$ and the symbols are drawn from the 16-QAM constellation. Notice that the FBMC/OQAM-based system with IIM-CBF 2 achieves a much better BER performance than its OFDMbased counterpart with the LoCCoBF algorithm [107]. These results further corroborate the fact that FBMC/OQAM is more immune to a lack of synchronization in the frequency domain compared to OFDM.

\section{Channel Estimation in MiMO-FBMC/OQAM SYSTEMS}

Acquiring CSI in FBMC/OQAM systems is a challenging task, significantly more difficult than in OFDM, due to the intrinsic interference effect. The challenge is intensified in MIMO systems, where the multi-antenna interference has also to be taken into account [14]. It is therefore not surprising that most of the research in FBMC/OQAM channel estimation has relied on the assumption of channels that are slowly varying in frequency and time, focusing mainly on model 2) in (26). The motivation behind this simplification is to formulate the problem in a way similar to OFDM. Of course, the similarity is only in the input-output relation appearance, since FBMC/OQAM also involves ISI/ICI. Moreover, this assumption may be quite inaccurate in communication environments involving, e.g., high data rate and/or mobility. In such cases, relying on the above assumption results in severe error floors at medium to high SNR values, which cancel the advantage of the FBMC/OQAM modulation over OFDM [108].

A number of training schemes and associated estimation methods have appeared in the literature, and can be categorized in preamble-based and scattered pilots-based ones. A recent review was given in [109], however only covering the SISO case. The MIMO case was reviewed in [108], where the focus was on preamble-based methods. These, of course, did not include recently reported methods that can also cope with highly frequency selective channels. This important case will be given a special emphasis here. In this section, we concisely present the state-of-the-art in channel estimation for MIMO-FBMC/OQAM. Scattered pilots-based techniques are of special interest in fast fading environments, where they allow tracking the channel variations throughout the frame (see, e.g., [110] and references therein). Since the MIMO designs presented in this paper are not suited to time-varying channels, this section only reviews preamble-based techniques. Blind methods (i.e., not relying on training signals) are also applicable in time invariant channels and are briefly discussed here.

\section{A. General assumptions}

The preamble is constructed so as to consist of a number of pilot FBMC/OQAM symbols, preceded and followed by one (or more ${ }^{2}$ ) symbol(s) of all zeros. This is to protect the pilots from being interfered by the unknown data (or control) samples of the previous and current frames, respectively. It must be noted, however, that in view of the inter-frame time gaps commonly used in wireless transmissions, only the guard(s) following the pilots can be necessary. This is the case considered here. Note that two FBMC/OQAM symbols (such as a pilot followed by a guard) last about one CPfree OFDM symbol and hence the training overheads in the two multicarrier schemes are comparable. For simplicity and analytical tractability, a fully loaded preamble will be considered, meaning that there are no inactive (virtual [114]) subcarriers at the edges of the signal spectrum. Two different pilot configurations for such preambles are considered: sparse, where only a number of isolated subcarriers carry pilots, with the rest of them being nulled, and full, with all frequencies being occupied with nonzero pilots [108]. These correspond to what is commonly called comb- and block-type pilot arrangements in OFDM, respectively [115].

Regarding the intrinsic interference, the most common assumption is that, with a well time-frequency localized pulse $p[n]$, interference contributions to a given frequency-time (FT) point $(q, k)$ only come from its first-order neighborhood [116], [117], namely $\Omega_{q, k}=\{(q \pm 1, k \pm 1),(q, k \pm 1),(q \pm 1, k)\}$ (see Fig. 7). It is important to note that the interference weights for the neighbors of $(q, k)$ can be a priori computed based on the employed prototype filter (analytical expressions were derived in [108]) and, for all $k$, enjoy the symmetries shown in the following

$$
\begin{array}{rrr}
(-1)^{q} \delta & -\beta & (-1)^{q} \delta \\
-(-1)^{q} \gamma & d_{q}[k] & (-1)^{q} \gamma \\
(-1)^{q} \delta & \beta & (-1)^{q} \delta
\end{array}
$$

with the horizontal direction corresponding to time and the vertical one to frequency as in Fig. 7. For example, the points $(q \pm 1, k)$ contribute $\pm j \beta d_{q \pm 1}[k]$ to the interference to $(q, k)$. The definition $\theta_{m}[k]=e^{j \frac{\pi}{2}(m+k)}$ is adopted throughout this section. The quantities in (95) are related to those described in (11) as follows: $\Gamma_{q, q}^{k}[-1]=j(-1)^{q} \gamma, \Gamma_{q-1, q}^{k}[-1]=$ $j(-1)^{q} \delta$, and $\Gamma_{q-1, q}^{k}[0]=j \beta$, where $\beta, \gamma, \delta$ are positive and smaller than one. Generally, $\beta, \gamma>\delta$, while in most cases $\gamma>\beta$ [108].

\section{B. Low frequency selective channels}

Under the above assumption and in line with model 2) (cf. Section II) in its simpler form corresponding to the channel

\footnotetext{
${ }^{2}$ One guard FBMC/OQAM symbol is not always sufficient and nonnegligible interference may still exist. Efficiently addressing the problem of the data interfering with the preamble is a crucial question that is still under investigation (see, e.g., [109]). Important related contributions include the memory preloading technique for transmitting/receiving a periodic preamble [111] and SISO channel estimation methods that are based on some iterative joint estimation/detection procedure for handling the unknown interference (cf. [108] for a review and some simulation results, and [112]). The problem of how to cope with the tails (due to a long prototype filter impulse response) of the transmitted burst is also relevant in this context [51], [111], [113].
} 
frequency response being invariant over a FT neighborhood, the output of the AFB at the $q$ th subcarrier and $k$ th time instant is given by (55) and (57), after compensating for the phase (i.e., multiplying with $\theta_{q}^{*}[k]$ ). The usefulness of (55) lies in its simplicity and similarity with what holds in OFDM and has thus been extensively used in the development of channel estimation and other signal processing techniques for FBMC/OQAM systems admitting such a simplification.

1) Interference approximation methods: In view of the above assumptions, if the immediate neighbors of $(q, k)$ carry training (hence known) symbols, one can compute an approximation of the interference term in (57), and hence construct the pseudo-pilot $\mathbf{c}_{q}[k]$. For a SISO system, the latter can be used to get a channel estimate in a way analogous to that for OFDM [115], namely ${ }^{3}$

$$
\hat{H}(q)=\frac{r_{q}[k]}{c_{q}[k]}=H(q)+\frac{\eta_{q}[k]}{c_{q}[k]},
$$

with $r_{q}[k] \triangleq \theta_{q}^{*}[k] y_{q}[k]$ and $\eta_{q}[k] \triangleq \theta_{q}^{*}[k] w_{q}[k]$.

This idea has been known as Interference Approximation Method (IAM) [117], [118] encompassing, under this name, a number of variants, each corresponding to a different preamble design. Those among them that have received a greater interest are the IAM schemes that aim at maximizing the magnitude of the pseudo-pilots in order to enhance the estimation accuracy, as seen in (96). This is achieved by so choosing the signs of the pilot symbols so that the symmetries (95) result in an increase of the pseudo-pilots magnitude. Note that in addition to the IAM schemes relying on a single pilot FBMC symbol, 3-symbol preambles for IAM have also been proposed and studied (with the aid of (95)), aiming at further increasing the pseudo-pilots strength and hence improving the estimation performance [108], [119], [120].

These methods were reviewed and tested in [108] for both SISO and MIMO systems. The MIMO case was first studied in [121], where it was proposed to construct the preambles for the $N_{T}$ antennas in the following way: for each transmit antenna, repeat the SISO preamble $N_{T}$ times, by also inserting sign changes that ensure orthogonality among the different antennas (similarly with MIMO-OFDM orthogonal training described in [122]). Fig. 21 depicts an example for the case $N_{T}=2$, using the IAM-C preamble (proved to be optimal in SISO channel estimation [108]). Each of the two antennas uses the SISO preamble twice, however with a sign reversal at the second pilot FBMC/OQAM symbol for the second antenna. Taking the structure of this preamble into account and recalling the assumption about the interference being mostly contributed by the first-order FT neighbors, it turns out that [108], [121]

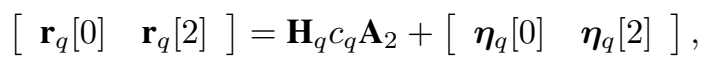

where $\mathbf{A}_{2}$ is the orthogonal matrix

$$
\mathbf{A}_{2}=\left[\begin{array}{rr}
1 & 1 \\
1 & -1
\end{array}\right]
$$

\footnotetext{
${ }^{3}$ In an idealized scenario where there is no noise, this is known as ideal channel estimation in the FBMC/OQAM literature [78], [116].
}

$$
\begin{array}{rrrr}
1 & 0 & 1 & 0 \\
-j & 0 & -j & 0 \\
-1 & 0 & -1 & 0 \\
j & 0 & j & 0 \\
1 & 0 & 1 & 0 \\
-j & 0 & -j & 0 \\
-1 & 0 & -1 & 0 \\
j & 0 & j & 0
\end{array}
$$

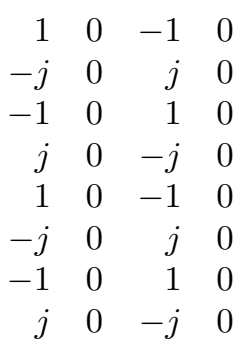

(b)
Figure 21. IAM-C preamble for a $2 \times X$ system, with (a) and (b) corresponding to the two transmit antennas. $M=8$. OQPSK modulation is assumed.

and $\left|c_{q}\right|=1+2 \beta$ for all $q$. An estimate of the channel matrix at subcarrier $q$ can then be computed as

$$
\begin{aligned}
& \hat{\mathbf{H}}_{q}=\left[\begin{array}{ll}
\mathbf{r}_{q}[0] & \mathbf{r}_{q}[2]
\end{array}\right] \frac{1}{c_{q}} \mathbf{A}_{2}^{-1} \\
& =\mathbf{H}_{q}+\frac{1}{2 c_{q}}\left[\begin{array}{ll}
\boldsymbol{\eta}_{q}[0] & \boldsymbol{\eta}_{q}[2]
\end{array}\right] \mathbf{A}_{2} \text {. }
\end{aligned}
$$

It is important to remark that the above is only valid when the channel frequency response can be seen as invariant over the first-order FT neighborhood of each pilot symbol (simplified model 2)) and can be easily generalized to any $N_{T}$ that is a power of two [108]. The idea behind this preamble construction lies in the fact that, due to the orthogonality of the matrix $\mathbf{A}_{2}$, the estimation noise power is again (as in the SISO case) exclusively controlled by the magnitude of the pseudopilot $c_{q}$. However, as explained in detail in [108], the above is not exact due to the fact that in practice there may exist a non-negligible interference between time instants 0 and 2 . The consequence of this is that the channels from the $N_{T}$ antennas are not all estimated with the same accuracy. Of course, this can be easily overcome, at an extra cost in the training overhead, if more than one guard symbols are placed among the repetitions of the IAM preamble. One must also note, about these IAM preambles, that their good performance comes at the cost of a high peak-to-average power ratio (PAPR) in the associated modulator outputs, as exemplified in [108]. This is due to their "deterministic" (periodic) structure.

2) The Pairs of Pilots method: This method, applicable in situations described by the simplified model 2) and originally proposed for SISO channels, stems from an alternative approach, that of solving a pair of equations (55) to compute the real and imaginary parts of the channel frequency response. In addition to being simpler, it does not explicitly depend on the employed prototype filter, provided, of course, (55) is satisfied. However, the method itself was developed on the basis of the zero noise assumption and can have a quite poor performance in practice. MIMO extensions, also exhibiting a bad performance, appeared in [123] and [108].

3) Sparse preambles: The MIMO IAM preambles described above are of duration proportional to the number of transmit antennas, $N_{T}$, and can thus entail a considerable loss in bandwidth efficiency. Shorter preambles, consisting of only one pilot FBMC/OQAM symbol and a guard one per antenna (independently of $N_{T}$ ), were reported in [123] and they are 
of the sparse type. One such example, known as frequencydivision multiplexing (FDM) [124], results from sharing the pilot subcarriers among the antennas and allows, for a given receive antenna, to separately estimate the channels from each of the transmit antennas. The frequency response values at the inactive frequencies are then found via interpolation in the frequency direction. This idea reappeared recently in [125], where it was shown to result in a significantly lower PAPR compared to the full preambles of [121]. We here recall from [108] a sparse scheme that was developed based on earlier work on orthogonal training design for MIMO-OFDM systems so as to be optimal in the MSE sense. Such a channel estimation approach was also adopted in [90]. The method that will be reviewed hereinafter provides an estimate of the channel impulse response for low frequency selective channels.

Assume, without loss of generality, that $N=\frac{M}{L_{\mathrm{ch}}}$ is an integer, not smaller than $2 N_{T}$. Consider then $N_{T}^{L_{\mathrm{ch}}}$ sets of $L_{\mathrm{ch}}$ pilots each, with the pilots in each set being placed at equispaced subcarriers and chosen to be equipowered. Let $q_{i} \in\{0,1, \ldots, N-1\}, i=1,2, \ldots, N_{T}$, be the userchosen position of the first pilot in the $i$ th pilot set. Thus, for each antenna, $N_{T} L_{\mathrm{ch}}$ pilots are placed at subcarriers $\left\{q_{i}, q_{i}+N, q_{i}+2 N, \ldots, q_{i}+\left(L_{\mathrm{ch}}-1\right) N\right\}, i=1,2 \ldots, N_{T}$. The rest of the subcarriers carry nulls. In view of the above conditions, $q_{i}$ 's can be so selected as to have at least one zero between two non-zero pilots, thus avoiding (most of) the interference among them. Then one can write the following input-output relation for the corresponding received signals:

$$
\underbrace{\left[\begin{array}{c}
\mathbf{r}^{\left(q_{1}\right)} \\
\vdots \\
\mathbf{r}^{\left(q_{N_{T}}\right)}
\end{array}\right]}_{\mathbf{r}}=\underbrace{\left[\begin{array}{ccc}
\mathbf{C}_{q_{1}}^{1} & \cdots & \mathbf{C}_{q_{1}}^{N_{T}} \\
\vdots & \ddots & \vdots \\
\mathbf{C}_{q_{N_{T}}}^{1} & \cdots & \mathbf{C}_{q_{N_{T}}}^{N_{N_{T}}}
\end{array}\right]}_{\mathbf{C}} \mathbf{h}+\underbrace{\left[\begin{array}{c}
\boldsymbol{\eta}^{\left(q_{1}\right)} \\
\vdots \\
\boldsymbol{\eta}^{\left(q_{N_{T}}\right)}
\end{array}\right]}_{\boldsymbol{\eta}},
$$

where $\mathbf{r}^{\left(q_{i}\right)}=\left[\begin{array}{llll}\mathbf{r}_{q_{i}}^{T}[0] & \mathbf{r}_{q_{i}+N}^{T}[0] & \cdots & \mathbf{r}_{q_{i}+\left(L_{\mathrm{ch}}-1\right) N}^{T}[0]\end{array}\right]^{T} \in$ $\mathbb{C}^{N_{R} L_{\mathrm{ch}} \times 1}$ and $\boldsymbol{\eta}^{\left(q_{i}\right)} \in \mathbb{C}^{N_{R} L_{\mathrm{ch}} \times 1}$ is similarly defined. The

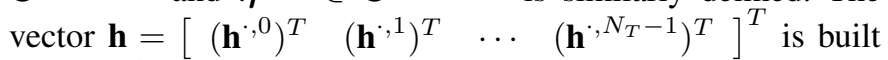
upon $\mathbf{h}^{\cdot, t} \in \mathbb{C}^{L_{\mathrm{ch}} N_{R} \times 1}$, which denotes the concatenation of the impulse responses of all channels from transmit antenna $t$ to all receive antennas. The matrices that constitute $\mathbf{C}_{q_{i}}^{t}=\mathbf{D}_{q_{i}}^{t}\left(\mathbf{F}_{L_{\mathrm{ch}}} \otimes \mathbf{I}_{N_{R}}\right) \mathbf{W}^{\left(q_{i}\right)} \in \mathbb{C}^{L_{\mathrm{ch}} N_{R} \times L_{\mathrm{ch}} N_{R}}$ are $\mathbf{F}_{L_{\mathrm{ch}}}$, the $L_{\mathrm{ch}} \times L_{\mathrm{ch}}$ submatrix of the $M$ th-order DFT matrix consisting of its first $L_{\mathrm{ch}}$ columns and every $N$ th of its rows, and

$$
\begin{aligned}
& \mathbf{D}_{q_{i}}^{t}=\operatorname{diag}\left\{d_{q_{i}}^{t}, d_{q_{i}+N}^{t}, \ldots, d_{q_{i}+N\left(L_{\mathrm{ch}}-1\right)}^{t}\right\} \otimes \mathbf{I}_{N_{R}} \\
& \mathbf{W}^{\left(q_{i}\right)}=\operatorname{diag}\left\{1, e^{-j \frac{2 \pi}{M} q_{i}}, \ldots, e^{-j \frac{2 \pi}{M} q_{i}\left(L_{\mathrm{ch}}-1\right)}\right\} \otimes \mathbf{I}_{N_{R}} .
\end{aligned}
$$

$d_{q_{i}}^{t}$ symbolizes the pilot transmitted on the $q_{i}$ th subcarrier, from the $t$ th transmit antenna. Note that $\boldsymbol{\eta}$ in (99) is white. It was shown in [108], [123] that $\mathbf{C}$ is unitary (hence leading to MSE-optimal least squares (LS) channel estimate from (99)) if $d_{q_{i}}^{t}=d_{q_{i}+N}^{t}=\cdots=d_{q_{i}+\left(L_{\mathrm{ch}}-1\right) N}^{t}$ for all $q_{i}$ and $t$, and the matrix

$$
\mathbf{D}=\left[\begin{array}{cccc}
d_{q_{1}}^{1} & d_{q_{1}}^{2} & \cdots & d_{q_{T}}^{N_{T}} \\
d_{q_{2}}^{1} & d_{q_{2}}^{2} & \cdots & d_{q_{2}}^{N_{T}} \\
\vdots & \vdots & \ddots & \vdots \\
d_{q_{N_{T}}}^{1} & d_{q_{N_{T}}}^{2} & \cdots & d_{q_{N_{T}}}^{N_{T}}
\end{array}\right]
$$

is unitary. Examples are given in [90], [108], [123].

4) Methods for distributed MIMO-FBMC/OQAM systems: A distributed multi-user MIMO-FBMC/OQAM setup is considered in [126] involving cooperating base stations with multiple antennas and assuming model 2) holds. The downlink channels are estimated without the use of pilots of any kind. Instead, properly designed small perturbations are applied in the transmitted signals, which are shown to indirectly provide CSI through their effects on the receive SINRs. The latter need to be estimated at the receivers and fed back to the basestations. This is the cost of not employing pilots, namely the time required to obtain good measurements and a (relatively low) feedback overhead. The effect of the chosen level of perturbation was studied in [126] in a number of interuser interference scenarios.

Downlink channel estimation in a distributed MIMOFBMC/OQAM context was also recently considered in [106] based on training preambles. The main goal of this work is to investigate the applicability and evaluate the comparative performance of two basic schemes for assigning pilot subcarriers to users: block versus interleaved configurations, with appropriate guards in each case to avoid multi-user interference. Model 2) in its simplified form is assumed to hold and the Linear Minimum Mean Squared Error (LMMSE) estimator is adopted in view of its robustness to the ill-conditioning associated with such subcarrier assignment schemes (SAS). Results demonstrate the robustness of FBMC/OQAM to lack of synchronization among the users (achieved with minimal guard bands) and reveals the relative advantages of the two SAS in various situations.

5) Blind MIMO-FBMC/OQAM channel estimation: The literature on (semi-)blind methods for FBMC/OQAM channel estimation is almost exclusively devoted to single-antenna systems (see, for example, [127], [128]) with the MIMO case only treated in [129]. ${ }^{4}$ The idea therein is to employ linear precoding that is so chosen as to allow the estimation of the channel directly from the estimated covariance matrix of the AFB output signal. Again, model 2) with the channel frequency response being invariant over a FT neighborhood is assumed. In that same work, an efficient implementation of the MIMO-FBMC/OQAM system is proposed, based on the well known decomposition of the FBMC/OQAM filter bank into two parallel filter banks, one for each of the two parts of the QAM signal [130].

\section{Highly frequency selective channels}

All of the channel estimation methods reviewed above rely on the assumptions underlying model 2), that is, they only apply to channels of relatively low delay spread. In the last few

\footnotetext{
${ }^{4}$ The method of [126] could be also included in this category, in view of the fact that it does not make use of any pilot information.
} 
years, there has been a significant progress towards the development of methods that are more robust to channel frequency selectivity (model 1)) but these only apply in SISO systems (cf. [109] for a review). The corresponding MIMO problem was only recently studied in [131], [132], through an extension to the MIMO setup of the results of [133]. Each antenna transmits a short full preamble, of one pilot FBMC/OQAM symbol $\mathbf{d}^{t} \in \mathbb{C}^{M \times 1}$, for $1 \leq t \leq N_{T}$, accompanied by the usual guard(s). These preambles are optimized to attain minimum channel estimation MSE. The method is based on a system formulation that makes absolutely no assumption on the channel frequency selectivity (apart from that commonly made in MIMO-OFDM, namely that $\left.M \geq N_{T} L_{\mathrm{ch}}\right)$ and expresses the received signal, for receive antenna $s$, as

$$
\mathbf{r}^{s}=\underbrace{\left[\begin{array}{llll}
\boldsymbol{\Gamma}\left(\mathbf{d}^{1}\right) & \boldsymbol{\Gamma}\left(\mathbf{d}^{2}\right) & \cdots & \boldsymbol{\Gamma}\left(\mathbf{d}^{N_{T}}\right)
\end{array}\right]}_{\boldsymbol{\Gamma}} \mathbf{h}^{s, \cdot}+\boldsymbol{\eta}^{s},
$$

where $\mathbf{h}^{s, \cdot} \in \mathbb{C}^{L_{\mathrm{ch}} N_{T} \times 1}$ is the concatenation of all channels associated to this antenna, $\mathbf{r}^{s}=\left[\begin{array}{lll}r_{0}^{s}[0] & \cdots & r_{M-1}^{s}[0]\end{array}\right]^{T}$ and $\boldsymbol{\eta}^{s} \in \mathbb{C}^{M \times 1}$ is similarly defined. The $M \times L_{\mathrm{ch}}$ matrix $\boldsymbol{\Gamma}\left(\mathbf{d}^{t}\right)$ has entries

$$
\begin{aligned}
{\left[\boldsymbol{\Gamma}\left(\mathbf{d}^{t}\right)\right]_{q, k}=} & \sum_{m=0}^{M-1} d_{m}^{t} \cdot e^{-j \frac{2 \pi}{M} q k} \cdot\left\{j^{m-q} e^{-j \frac{2 \pi}{M}(m-q)(k-D)}\right. \\
& \left.\sum_{l=k}^{L-1} p[l-k] p[l] e^{j \frac{2 \pi}{M}(m-q) l}\right\} \\
\triangleq & \sum_{m=0}^{M-1} d_{m}^{t} w_{M}^{q k}\left[\mathbf{G}_{k}\right]_{q, m}
\end{aligned}
$$

$q=0,1, \ldots, M-1, k=0,1, \ldots, L_{\mathrm{ch}}-1$, and can be seen to be equal to $\left[\begin{array}{llll}\mathbf{G}_{0} & \mathbf{W G}_{1} & \cdots & \mathbf{W}^{L_{\mathrm{ch}}-1} \mathbf{G}_{L_{\mathrm{ch}}-1}\end{array}\right]\left(\mathbf{I}_{L_{\mathrm{ch}}} \otimes \mathbf{d}^{t}\right)$ with $\mathbf{W}=\operatorname{diag}\left(w_{M}^{q}\right)_{q=0}^{M-1}$ and the matrices $\mathbf{G}_{k}$ built from the prototype filter as in (103) [133]. It is of interest to observe the physical meaning of the $k$ th column of $\boldsymbol{\Gamma}\left(\mathbf{d}^{t}\right)$ : it represents the response of the transmultiplexer to the input $\mathbf{d}^{t}$ for a channel equal to a delay of $k$ samples [133]. Moreover, the covariance matrix of $\boldsymbol{\eta}^{s}$ is given by $\mathbf{C}_{\boldsymbol{\eta}}=N_{0} \mathbf{G}_{0}$ and this is taken into account in computing the Gauss-Markov channel estimate from (102), namely

$$
\hat{\mathbf{h}}^{s, \cdot}=\left(\boldsymbol{\Gamma}^{H} \mathbf{C}_{\boldsymbol{\eta}}^{-1} \boldsymbol{\Gamma}\right)^{-1} \boldsymbol{\Gamma}^{H} \mathbf{C}_{\boldsymbol{\eta}}^{-1} \mathbf{r}^{s} .
$$

Let $\lambda_{k, i}, i=0,1, \ldots, M-1$, denote the $i$ th DFT coefficient of the first row of $\mathbf{G}_{k} \cdot{ }^{5}$ MSE-optimal preambles with a constraint

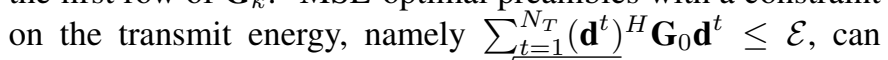
be constructed as follows: $\mathbf{d}^{t}=\sqrt{\frac{\mathcal{E}}{N_{T} \lambda_{0, i_{t}}}} \mathbf{f}_{i_{t}+1}$, where $\mathbf{f}_{i_{t}+1}$ is the $\left(i_{t}+1\right)$ st column of the $M$ th-order DFT matrix and $0 \leq i_{t} \leq M-1$ is chosen to minimize $\lambda_{0, i} \sum_{k=1}^{L_{\mathrm{ch}}-1} \frac{\lambda_{0,((k+i))_{M}}}{\lambda_{k, i}^{2}}$, with $((\cdot))_{M}$ denoting modulo $M$. Moreover, once $i_{t}$ has been determined, the possible values for $i_{r}, r \neq t$, exclude the set $\left\{i_{t},\left(\left(i_{t} \pm 1\right)\right)_{M},\left(\left(i_{t} \pm 2\right)\right)_{M}, \ldots,\left(\left(i_{t} \pm\left(L_{\mathrm{ch}}-1\right)\right)\right)_{M}\right\}$. The optimal preambles assume an even simpler form when restricted to be strictly OQAM, i.e., with real valued symbols,

\footnotetext{
${ }^{5}$ This corresponds, in fact, to the eigenvalues of $\mathbf{G}_{k}$ in view of the circulant structure of these matrices [131], [133].
}

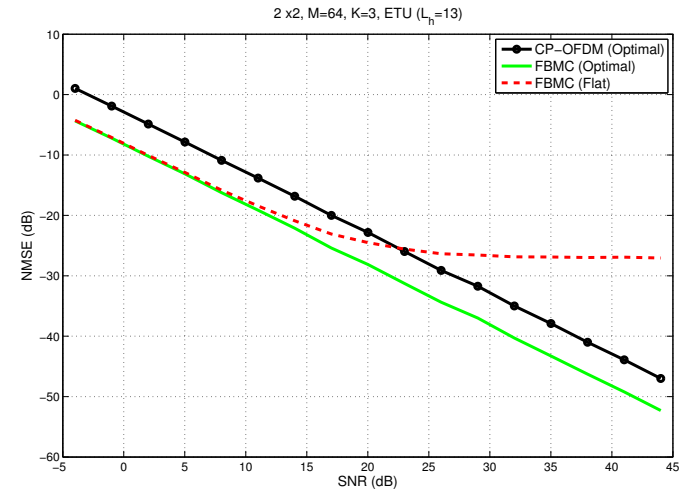

Figure 22. Estimation performance of preamble-based methods for $2 \times 2$ Extended Typical Urban (ETU) channels. Filter banks designed as in [44] with $M=64$ and $K=3$ were employed.

$\mathbf{d}^{t} \in \mathbb{R}^{M \times 1}$. Indeed, for $N_{T}=2$ transmit antennas, optimal pilots are all equal for one antenna and all equal with alternating signs for the other. It should be also noted that the channel estimation procedure is greatly simplified when optimal preambles are employed. For details, see [132]. An example of the performance of this method as compared to that of the MSE-optimal MIMO-OFDM method of [134] is given in Fig. 22, for channels exhibiting a significant frequency selectivity relatively to the filter bank size (Scenario 1). The result of assuming flat subchannels (according to the simplified model 2)) in the preamble and estimator design of [131] is also shown. As it is typical in methods that rely on the flat subchannel model, a severe error floor is observed at above medium SNR values, due to the fact that the residual intrinsic interference becomes more apparent in weak noise regimes.

\section{CONClusions AND Future Research}

Motivated by key challenges in the design of the air interface for communication systems including $5 \mathrm{G}$ and broadband PMR networks among others, the signal processing problems underlying the application of FBMC/OQAM in multi-antenna settings were considered in this paper, with emphasis given to the most recent advances in this area. The adoption of FBMC/OQAM is dictated by the capability of this modulation to support orthogonal transmission with maximum spectral efficiency while allowing a flexible use of the spectrum. However, as explained in this paper, the full potential of FBMC/OQAM cannot be straightforwardly reached when it is combined with MIMO technology. One cannot in general rely on solutions developed for MIMO-OFDM and hence FBMCspecific signal processing techniques have to be devised and employed. In this paper, an extensive overview of known solutions to the MIMO-FBMC/OQAM signal processing problems was presented, in a comprehensive manner and using a unified notational framework.

It is well known that when CSI is available only at the receive side, the maximum rate is obtained by applying plain MIMO spatial multiplexing. In this case, the least complex solution to recover the original data symbols at the receiver consists in detecting the symbols separately, after performing 
equalization. In order to combat the channel frequency selectivity, especially when the channel exhibits a large delay spread, three alternatives were reviewed. The first one tries to compensate for the channel distortion by combining multiple AFB stages so that the combined response approximates the inverse of the channel frequency response. The second and third approaches seek to turn the end-to-end system into a parallel transmission system free of interference, in either the time or the frequency domain. All three approaches achieve similar performance and succeed in mitigating the channel frequency selectivity. The overall system performance is increased if the symbols are jointly detected. However, in the FBMC/OQAM context, this implies exploring a huge number of symbol combinations due to ISI and ICI. In order to reduce the complexity of this family of algorithms, the size of the alphabet can be reduced via interference cancellation procedures. In this case, two iterations are required at least, namely one to estimate the interference and another one to estimate the symbols after suppressing the unwanted signals. Interestingly, the best performance is achieved by completely removing ICI and allowing two ISI terms to be considered as useful information.

This paper also delved into the design of MIMO precoding and decoding matrices when CSI is known at both ends of the link. It was demonstrated that the solutions originally conceived for OFDM cause interference when FBMC/OQAM is adopted for signal modulation, unless the channel is frequency non-selective over at least three consecutive subcarriers. To extend the use of MIMO under strong frequency selectivity, the multi-stage approach has been used to design frequency selective precoders and equalizers, so that the global response is ideal. Sticking to the single-stage architecture, which corresponds to the conventional FBMC/OQAM transceiver, the detrimental effects of the channel can be also overcome, at the same time that the rich scattering of the environment is exploited, by employing multi-tap equalizers and single-tap precoders. Since the optimal joint design is very intricate, the use of bounds was proposed to relax the problem. Satisfactory performance is achieved only if $N_{T} \leq N_{R}$. Thus, it remains as an open problem to find multi-tap designs that achieve competitive results for any multi-antenna configuration. The multi-stage approach does not suffer from this dimensionality constraint, but its complexity grows with the number of stages. Another aspect that deserves further investigation in highly frequency selective channels is the design of the transmitter and the receiver in the multi-user case [58]. This is a definitely relevant topic, because existing solutions decouple the transmitter and the receiver design, which does not lead to the optimal solution.

As it is the case also for single-antenna systems, MIMO designs are simplified in the presence of low frequency selective channels. In this scenario, precoders and equalizers can be designed to project desired and unwanted terms onto orthogonal subspaces, facilitating interference suppression. This idea is extended to the multi-user context to adapt the BD concept and the STHP to FBMC/OQAM systems, in order to achieve interference-free data multiplexing. However, again this strategy is only applicable in the case where $N_{R} \leq N_{T}$.
To overcome this constraint, algorithms of an iterative nature are required. For the moment, it is unknown how to achieve the same performance with non-iterative solutions.

To simplify the symbol detection in FBMC/OQAM systems, it is convenient to suppress the interference through the exploitation of the PR property. Towards this end, the equivalent MIMO channel, which includes precoding and decoding matrices, has to be real-valued. Some interesting ideas to make the channel real are highlighted in [135]. It is shown in this paper that this task is facilitated when precoders or decoders are chosen in the real field. Sometimes this choice is not theoretically supported but it relies on computer simulations. Therefore, a theoretical framework for predicting under what conditions precoders and equalizers shall be constrained to be real-valued still needs to be developed. Based on that, it seems reasonable to question if it is better to rely on orthogonal modulations or work instead with non-orthogonal designs. This area of research is not fully explored and the question remains open. To shed light into this topic, it would be necessary as future work to evaluate the spectral efficiency, the complexity order and the sensitivity to synchronization errors of different MIMO-FBMC schemes.

Another problem that still needs to be better understood is the effect of mobility on the performance of FBMC/OQAM. For mildly time selective scenarios, it is possible to generalize the study in [70] and [69] to the case where there is a difference between the real channel and the one used in the construction of the precoder/equalizer. This difference could account for either channel estimation errors or time variations due to the Doppler effect. The asymptotic approach in [69], [70] could shed some light on the performance of the FBMC/OQAM system under these non-ideal conditions and could establish a formal comparison with classical CP-OFDM in terms of robustness against these effects.

On the other hand, the behavior of FBMC/OQAM in massive MIMO contexts is still far from being fully understood. In particular, the self-equalization effect reported in [55] still needs to be explored from the analytical point of view. It is our belief that random matrix theory tools in combination with the asymptotic approach in [69] could well provide a formal characterization of the self-equalization effect in large-scale MIMO settings and lead to useful results.

CSI, which is required in one way or another in the previous techniques, is made available through channel estimation, which has to face its own challenges in FBMC/OQAM-based systems. This part was also reviewed in this paper, restricting attention to those techniques that are most relevant in the present context. Training schemes and associated estimation techniques were presented in a concise manner, for both low and highly frequency selective channels, and optimal preamble design was also considered. Emphasis was given to the highly frequency selective case, since it is where the most recent advances in this area refer to. Open problems and related future research directions (some of which have been alluded to in the paper) include (but are not limited to): preamble design and channel estimation with virtual (inactive) subcarriers (a research problem that is still of interest in OFDM as well [136]); optimization of preambles longer 
than one pilot FBMC symbol ${ }^{6}$; exploitation of the channel sparsity (common in wireless transmission) for performance enhancement/pilot savings; channel estimation in multiuser settings involving highly selective channels; more advanced (semi-)blind estimation schemes, with possible applications in addressing the pilot contamination problem in massive MIMOFBMC/OQAM systems.

The material presented in this paper confirms that the application of FBMC/OQAM to a MIMO context is becoming mature. Although there are still several interesting open problems that need to be addressed, recent advances seem to place MIMO-FBMC/OQAM among the top candidates for implementing a powerful and versatile air interface as required in next generation communication systems.

\section{ACKNOWLEDGMENT}

This work has received funding from the Spanish Government under projects TEC2014-59255-C3-1-R and Red ARCO 5G TEC2014-56469-REDT and from the Catalan Government under grant 2014SGR1567.

\section{REFERENCES}

[1] J. Andrews, S. Buzzi, W. Choi, S. Hanly, A. Lozano, A. Soong, and J. Zhang, "What will 5G be?" IEEE J. Sel. Areas Commun., vol. 32, no. 6, pp. 1065-1082, June 2014.

[2] P. Banelli, S. Buzzi, G. Colavolpe, A. Modenini, F. Rusek, and A. Ugolini, "Modulation formats and waveforms for 5G networks: Who will be the heir of OFDM?" IEEE Signal Process. Mag., vol. 31, no. 6, pp. 80-93, Nov. 2014.

[3] "Evolved Universal Terrestrial Radio Access (E-UTRA); Physical channels and modulation (3GPP TS 36.211 version 12.3.0 Release 12)."

[4] WMF-T23-001-R022v02, "Air Interface Specifications-Mobile System Profile Release 2.2," WiMAX Forum. [Online]. Available: http://www.wimaxforum.org/resources/technical-specifications

[5] "IEEE Standard for Information technology- Local and metropolitan area networks- Specific requirements- Part 11: Wireless LAN Medium Access Control (MAC)and Physical Layer (PHY) Specifications Amendment 5: Enhancements for Higher Throughput," IEEE Std 802.11n-2009 (Amendment to IEEE Std 802.11-2007 as amended by IEEE Std 802.11k-2008, IEEE Std 802.11r-2008, IEEE Std 802.11y2008, and IEEE Std 802.11w-2009), pp. 1 -565, 292009.

[6] "Digital Video Broadcasting (DVB); Frame structure channel coding and modulation for a second generation digital terrestrial television broadcasting system (DVB-T2).” $\quad$ ETSI EN 302755 V1.2.1 (201010).

[7] "Radio Broadcasting Systems; Digital Audio Broadcasting (DAB) to mobile, portable and fixed receivers." ETSI EN 300401 V1.4.1 (200601).

[8] K. Panta and J. Armstrong, "Spectral analysis of OFDM signals and its improvement by polynomial cancellation coding," IEEE Trans. Consum. Electron., vol. 49, no. 4, pp. 939-943, Nov. 2003.

[9] S. Brandes, I. Cosovic, and M. Schnell, "Sidelobe suppression in OFDM systems by insertion of cancellation carriers," in IEEE 62nd Vehicular Technology Conference, Dallas, USA, Sept. 2005.

[10] I. Cosovic, S. Brandes, and M. Schnell, "Subcarrier weighting: a method for sidelobe suppression in OFDM systems," IEEE Commun. Lett., vol. 10, no. 6, pp. 444-446, June 2006.

[11] A. Sahin and H. Arslan, "Edge windowing for OFDM based systems," IEEE Commun. Lett., vol. 15, no. 11, pp. 1208-1211, November 2011.

[12] A. Loulou, S. Gorgani, and M. Renfors, "Enhanced OFDM techniques for fragmented spectrum use," in Future Network and Mobile Summit, Lisbon, Portugal, July 2013.

[13] P. P. Vaidyanathan, Multirate Systems and Filter Banks, ser. PrenticeHall signal processing series. Englewood Cliffs, N.J. Prentice Hall, 1993.

[14] B. Farhang-Boroujeny, "OFDM versus filter bank multicarrier," IEEE Signal Process. Mag., vol. 28, no. 3, pp. 92-112, May 2011.

${ }^{6}$ Results in this direction were recently reported in [137] for SISO systems.
[15] H. Feichtinger and T. Ströhmer, Eds., Advances in Gabor Analysis. Birkhauser Boston, 2003.

[16] "PHYsical layer for DYnamic spectrum AccesS and cognitive radio (PHYDYAS)." [Online]. Available: http://www.ict-phydyas.org/

[17] "Enhanced Multicarrier techniques for Professional Ad-hoc and Cell-based communications (EMPhAtiC)." [Online]. Available: http://www.ict-emphatic.eu/

[18] "5th Generation Non-Orthogonal Waveforms for asynchronous signaling (5GNOW)." [Online]. Available: http://www.5gnow.eu/

[19] "Mobile and wireless communications Enablers for the Twentytwenty Information Society (METIS)." [Online]. Available: https://www.metis2020.com/

[20] "Flexible Air iNTerfAce for Scalable service delivery wiThin wIreless Communication networks of the 5th Generation (FANTASTIC 5G)." [Online]. Available: http://fantastic5g.eu/

[21] “The 5G Infrastructure Public-Private Partnership (5G PPP)." [Online]. Available: http://5g-ppp.eu/

[22] Q. Li, H. Niu, A. Papathanassiou, and G. Wu, "5G network capacity: Key elements and technologies," IEEE Veh. Technol. Mag., vol. 9, no. 1, pp. 71-78, March 2014.

[23] J. Dommel, G. Boccolini, L. Raschkowski, S. Jaeckel, L. Thiele, T. Haustein, and N. Gonzalez Prelcic, " $5 \mathrm{G}$ in space: PHY-layer design for satellite communications using non-orthogonal multi-carrier transmission," in Advanced Satellite Multimedia Systems Conference and the 13th Signal Processing for Space Communications Workshop (ASMS/SPSC), Livorno, Italy, Sept. 2014.

[24] B. Le Floch, M. Alard, and C. Berrou, "Coded orthogonal frequency division multiplex," Proc. IEEE, vol. 83, no. 6, pp. 982-996, June 1995.

[25] P. Siohan, C. Siclet, and N. Lacaille, "Analysis and design of OFDM/OQAM systems based on filterbank theory," IEEE Trans. Signal Process., vol. 50, no. 5, pp. 1170-1183, May 2002.

[26] B. Saltzberg, "Performance of an efficient parallel data transmission system," IEEE Trans. Commun. Technol., vol. 15, no. 6, pp. 805-811, Dec. 1967

[27] L. Baltar, F. Schaich, M. Renfors, and J. Nossek, "Computational complexity analysis of advanced physical layers based on multicarrier modulation," in Future Network Mobile Summit (FutureNetw), Warsaw, Poland, June 2011.

[28] V. Vakilian, T. Wild, F. Schaich, S. ten Brink, and J.-F. Frigon, "Universal-filtered multi-carrier technique for wireless systems beyond LTE," in IEEE Globecom Workshops (GC Wkshps), Atlanta, USA, Dec. 2013.

[29] T. Wild, F. Schaich, and Y. Chen, "5G air interface design based on Universal Filtered (UF-)OFDM," in 19th International Conference on Digital Signal Processing (DSP), Kowloon, Hong Kong, Aug 2014.

[30] G. Fettweis, M. Krondorf, and S. Bittner, "GFDM - Generalized Frequency Division Multiplexing," in IEEE 69th Vehicular Technology Conference, Barcelona, Spain, 2009.

[31] N. Michailow, M. Matthe, I. Gaspar, A. Caldevilla, L. Mendes, A. Festag, and G. Fettweis, "Generalized frequency division multiplexing for 5th generation cellular networks," IEEE Trans. Commun., vol. 62, no. 9, pp. 3045-3061, Sept 2014.

[32] A. Farhang, N. Marchetti, and L. Doyle, "Low complexity GFDM receiver design: A new approach," in IEEE International Conference on Communications (ICC), London, UK, June 2015.

[33] B. Farhang-Boroujeny and H. Moradi, "Derivation of GFDM based on OFDM principles," in IEEE International Conference on Communications (ICC), London, UK, June 2015.

[34] G. Cherubini, E. Eleftheriou, and S. Ölçer, "Filtered multitone modulation for VDSL," in Global Telecommunications Conference, Rio de Janeiro, Brazil, Dec. 1999.

[35] — , "Filtered multitone modulation for very high-speed digital subscriber lines," IEEE J. Sel. Areas Commun., vol. 20, no. 5, pp. 10161028, June 2002.

[36] A. Tonello and M. Girotto, "Cyclic block filtered multitone modulation,” EURASIP Journal on Advances in Signal Processing, vol. 2014, no. $1,2014$.

[37] H. Lin and P. Siohan, "Multi-carrier modulation analysis and WCPCOQAM proposal," EURASIP Journal on Advances in Signal Processing, vol. 2014, no. 1, 2014.

[38] D. Chen, X.-G. Xia, T. Jiang, and X. Gao, "Properties and power spectral densities of CP based OQAM-OFDM systems," IEEE Trans. Signal Process., vol. 63, no. 14, pp. 3561-3575, July 2015.

[39] D. Kong, X. G. Xia, T. Jiang, and X. Gao, "Channel estimation in CPOQAM-OFDM systems," IEEE Trans. Signal Process., vol. 62, no. 21, pp. 5775-5786, Nov 2014. 
[40] H. Lin and P. Siohan, "An advanced multi-carrier modulation for future radio systems," in IEEE International Conference on Acoustics, Speech and Signal Processing (ICASSP), Florence, Italy, May 2014.

[41] I. Estella, A. Pascual-Iserte, and M. Payaro, "OFDM and FBMC performance comparison for multistream MIMO systems," in Proc. Future Network and Mobile Summit, Florence, Italy, June 2010.

[42] R. W. Chang, "Synthesis of band-limited orthogonal signals for multichannel data transmission," The Bell System Technical Journal, vol. 45, no. 10 , pp. 1775-1796, Dec 1966.

[43] H. Bölcskei, "Orthogonal frequency division multiplexing based on offset QAM," in Advances in Gabor Analysis, H. Feichtinger and T. Ströhmer, Eds. Birkhauser Boston, 2003, pp. 321-352.

[44] M. G. Bellanger, "Specification and design of a prototype filter for filter bank based multicarrier transmission," in IEEE Int'l Conf. Acoustics, Speech, and Signal Process. (ICASSP-2001), Salt Lake City, Utah, USA, May 2011.

[45] H. Bölcskei, P. Duhamel, and R. Hleiss, "Design of pulse shaping OFDM/OQAM systems for high data-rate transmission over wireless channels," in IEEE International Conference on Communications, Vancouver, Canada, June 1999.

[46] A. Viholainen, T. Ihalainen, T. H. Stitz, M. Renfors, and M. Bellanger, "Prototype filter design for filter bank multicarrier transmission," in Proc. European Signal Processing Conference (EUSIPCO-2009), Glasgow, Scotland, Aug. 2009.

[47] D. Chen, D. Qu, T. Jiang, and Y. He, "Prototype filter optimization to minimize stopband energy with NPR constraint for filter bank multicarrier modulation systems," IEEE Trans. Signal Process., vol. 61, no. 1, pp. 159-169, Jan. 2013.

[48] A. Sahin, I. Guvenc, and H. Arslan, "A survey on multicarrier communications: Prototype filters, lattice structures, and implementation aspects," IEEE Commun. Surveys Tuts., vol. 16, no. 3, pp. 1312-1338, Third Quarter 2014.

[49] M. Bellanger, "Efficiency of filter bank multicarrier techniques in burst radio transmission," in IEEE Global Telecommunications Conference, Miami, Florida, USA, Dec. 2010.

[50] T. J. F. Wang, D. Qu and B. Farhang-Boroujeny, "Tail shortening by virtual symbols in FBMC-OQAM signals," in IEEE Signal Processing and Signal Processing Education Workshop, Salt Lake City, USA, Aug. 2015.

[51] M. J. Abdoli, M. Jia, and J. Ma, "Weighted circularly convolved filtering in OFDM/OQAM," in IEEE 24th International Symposium on Personal Indoor and Mobile Radio Communications (PIMRC), London, UK, Sept. 2013.

[52] J. McWhirter, P. Baxter, T. Cooper, S. Redif, and J. Foster, "An EVD algorithm for para-Hermitian polynomial matrices," IEEE Trans. Signal Process., vol. 55, no. 5, pp. 2158-2169, May 2007.

[53] "3rd Generation Partnership Project; Technical Specification Group Radio Access Network; Evolved Universal Terrestrial Radio Access (E-UTRA); User Equipment (UE) radio transmission and reception; (Release 8)." 3GPP TR 36.803v1.1.0.

[54] A. Farhang, N. Marchetti, F. Figueiredo, and J. Miranda, "Massive MIMO and waveform design for 5th generation wireless communication systems," in 2014 1st International Conference on $5 G$ for Ubiquitous Connectivity (5GU), Levi, Finland, Nov. 2014.

[55] A. Farhang, N. Marchetti, L. Doyle, and B. Farhang-Boroujeny, "Filter bank multicarrier for massive MIMO," in IEEE 80th Vehicular Technology Conference (VTC Fall), Vancouver, Canada, Sept. 2014.

[56] B. Picinbono and P. Chevalier, "Widely linear estimation with complex data," IEEE Trans. Signal Process., vol. 43, no. 8, pp. 2030-2033, Aug. 1995.

[57] M. Bellanger, "Specification and design of a prototype filter for filter bank based multicarrier transmission," in IEEE International Conference on Acoustics, Speech, and Signal Processing, Salt Lake City, USA, May 2001.

[58] D. Gregoratti and X. Mestre, "Uplink FBMC/OQAM-based multiple access channel: Distortion analysis under strong frequency selectivity," IEEE Trans. Signal Process., 2016.

[59] H. Saeedi-Sourck, Y. Wu, J. W. M. Bergmans, S. Sadri, and B. FarhangBoroujeny, "Complexity and performance comparison of filter bank multicarrier and OFDM in uplink of multicarrier multiple access networks," IEEE Trans. Signal Process., vol. 59, no. 4, pp. 1907-1912, April 2011.

[60] M. Morelli, C. C. J. Kuo, and M. O. Pun, "Synchronization techniques for orthogonal frequency division multiple access (OFDMA): A tutorial review," Proc. IEEE, vol. 95, no. 7, pp. 1394-1427, July 2007.

[61] E. G. Larsson and P. Stoica, Space-Time Block Coding for Wireless Communications. Cambridge University Press, 2008.
[62] C. Lélé, P. Siohan, and R. Legouable, "The Alamouti scheme with CDMA-OFDM/OQAM.” EURASIP J. Adv. Sig. Proc., vol. 2010, 2010.

[63] R. Zakaria and D. L. Ruyet, "Intrinsic interference reduction in a filter bank-based multicarrier using QAM modulation," Physical Communication, vol. 11, pp. $15-24,2014$.

[64] R. Zakaria and D. Ruyet, "On interference cancellation in Alamouti coding scheme for filter bank based multicarrier systems," in Proceedings of the Tenth International Symposium on Wireless Communication Systems (ISWCS 2013), Ilmenau, Germany, Aug. 2013.

[65] M. Renfors, T. Ihalainen, and T. Stitz, "A block-Alamouti scheme for filter bank based multicarrier transmission," in European Wireless Conference (EW), Lucca, Italy, April 2010.

[66] H. Lin, C. Lélé, and P. Siohan, "A pseudo Alamouti transceiver design for OFDM/OQAM modulation with cyclic prefix," in IEEE 10th Workshop on Signal Processing Advances in Wireless Communications, Perugia, Italy, June 2009.

[67] R. Zakaria and D. Le Ruyet, "A novel filter-bank multicarrier scheme to mitigate the intrinsic interference: application to MIMO systems," IEEE Trans. Wireless Commun., vol. 11, no. 3, pp. 1112-1123, March 2012.

[68] B. M. Kurkoski, "Towards efficient detection of two-dimensional intersymbol interference channels," IEICE Trans. Fundam. Electron. Commun. Comput. Sci., vol. E91-A, no. 10, pp. 2696-2703, Oct. 2008.

[69] X. Mestre and D. Gregoratti, "Parallelized structures for MIMO FBMC under strong channel frequency selectivity," IEEE Trans. Signal Process., vol. 64, no. 5, pp. 1200-1215, March 2016.

[70] X. Mestre, M. Majoral, and S. Pfletschinger, "An asymptotic approach to parallel equalization of filter bank multicarrier signals," IEEE Trans. Signal Process., vol. 61, no. 14, pp. 3592-3606, July 2013.

[71] T. Ihalainen, T. H. Stitz, M. Rinne, and M. Renfors, "Channel equalization in filter bank based multicarrier modulation for wireless communications," EURASIP J. Appl. Signal Process., vol. 2007, no. 1, pp. 140-140, Jan. 2007.

[72] D. Waldhauser, L. Baltar, and J. Nossek, "MMSE subcarrier equalization for filter bank based multicarrier systems," in IEEE 9th Workshop on Signal Processing Advances in Wireless Communications, Recife, Brazil, July 2008.

[73] A. Ikhlef and J. Louveaux, "Per subchannel equalization for MIMO FBMC/OQAM systems," in IEEE Pacific Rim Conference on Communications, Computers and Signal Processing, Victoria, Canada, Aug. 2009.

[74] T. Ihalainen, A. Ikhlef, J. Louveaux, and M. Renfors, "Channel equalization for multi-antenna FBMC/OQAM receivers," IEEE Trans. Veh. Technol., vol. 60, no. 5, pp. 2070-2085, June 2011.

[75] M. Bellanger, "FS-FBMC: An alternative scheme for filter bank based multicarrier transmission," in 5th International Symposium on Communications Control and Signal Processing (ISCCSP), Rome, Italy, May 2012.

[76] M. Borgerding, "Turning overlap-save into a multiband mixing, downsampling filter bank," IEEE Signal Process. Mag., vol. 23, no. 2, pp. 158-161, March 2006.

[77] A. Aminjavaheri, A. Farhang, N. Marchetti, L. Doyle, and B. FarhangBoroujeny, "Frequency spreading equalization in multicarrier massive MIMO," in IEEE International Conference on Communication (ICC), London, UK, June 2015.

[78] M. El Tabach, J.-P. Javaudin, and M. Hélard, "Spatial data multiplexing over OFDM/OQAM modulations," in IEEE International Conference on Communications, Glasgow, Scotland, June 2007.

[79] R. Zakaria, D. Le Ruyet, and M. Bellanger, "Maximum likelihood detection in spatial multiplexing with FBMC," in European Wireless Conference (EW-2010), Lucca, Italy, April 2010.

[80] Y. Cheng and M. Haardt, "Widely Linear Processing in MIMO FBMC/OQAM Systems," in Proceedings of the Tenth International Symposium on Wireless Communication Systems, Ilmenau, Germany, Aug. 2013.

[81] R. Zakaria and D. Le Ruyet, "Partial ISI cancellation with Viterbi detection in MIMO filter-bank multicarrier modulation," in 8th International Symposium on Wireless Communication Systems (ISWCS), Aachen, Germany, Nov. 2011.

[82] H. Shen, B. Li, M. Tao, and X. Wang, "MSE-based transceiver designs for the MIMO interference channel," IEEE Transactions on Wireless Communications, vol. 9, no. 11, pp. 3480-3489, Nov. 2010.

[83] F. Sun and E. de Carvalho, "A leakage-based MMSE beamforming design for a MIMO interference channel," IEEE Signal Process. Lett., vol. 19 , no. 6 , pp. 368-371, June 2012.

[84] S. Shi, M. Schubert, and H. Boche, "Downlink MMSE transceiver optimization for multiuser MIMO systems: duality and sum-MSE 
minimization," IEEE Trans. Signal Process., vol. 55, no. 11, pp. 54365446, Nov 2007.

[85] O. D. Candido, L. G. Baltar, A. Mezghani, and J. A. Nossek, "SIMO/MISO MSE-duality for multi-user FBMC with highly frequency selective channels," in Proceedings of 19th International ITG Workshop on Smart Antennas, Ilmenau, Germany, March 2015.

[86] A. I. Pérez-Neira and M. Caus, "Dimensionality constraints imposed by highly frequency selective channels on MIMO-FBMC/OQAM systems," in 19th International ITG Workshop on Smart Antennas (WSA), Ilmenau, Germany, March 2015.

[87] M. Caus and A. Pérez-Neira, "Multi-stream transmission for highly frequency selective channels in MIMO-FBMC/OQAM systems," IEEE Trans. Signal Process., vol. 62, no. 4, pp. 786-796, Feb. 2014.

[88] S.-D. Wang, T.-S. Kuo, and C.-F. Hsu, "Trace bounds on the solution of the algebraic matrix Riccati and Lyapunov equation," IEEE Trans. Autom. Control, vol. 31, no. 7, pp. 654-656, July 1986.

[89] D. Palomar, J. Cioffi, and M.-A. Lagunas, "Joint Tx-Rx beamforming design for multicarrier MIMO channels: a unified framework for convex optimization," IEEE Trans. Signal Process., vol. 51, no. 9, pp. 2381-2401, Sept. 2003.

[90] M. Caus and A. Pérez-Neira, "Transmitter-receiver designs for highly frequency selective channels in MIMO FBMC systems," IEEE Trans. Signal Process., vol. 60, no. 12, pp. 6519-6532, Dec. 2012.

[91] M. Caus and A. I. Pérez-Neira, "Experimental performance bounds of MIMO-FBMC/OQAM systems," in 20th European Wireless Conference, Barcelona, Spain, May 2014.

[92] _ _ "Comparison of linear and widely linear processing in MIMOFBMC systems," in Proceedings of the Tenth International Symposium on Wireless Communication Systems (ISWCS 2013), Ilmenau, Germany, Aug. 2013.

[93] —- "Multi-stream transmission in MIMO-FBMC systems," in Proc. IEEE International Conference on Acoustics, Speech and Signal Processing (ICASSP-2013), Vancouver, Canada, May 2013.

[94] Q. H. Spencer, A. L. Swindlehurst, and M. Haardt, "Zero-forcing methods for downlink spatial multiplexing in multiuser MIMO channels," IEEE Trans. Signal Process., vol. 52, no. 2, pp. 461-471, Feb. 2004.

[95] Y. Cheng, P. Li, and M. Haardt, "Coordinated beamforming in MIMO FBMC/OQAM systems," in Proc. IEEE Int. Conference on Acoustics, Speech, and Signal Processing (ICASSP), May 2014, pp. Florence, Itlay.

[96] - "Intrinsic interference mitigating coordinated beamforming for the FBMC/OQAM based downlink," EURASIP Journal on Advances in Signal Processing, May 2014.

[97] F. Horlin, J. Fickers, T. Deleu, and J. Louveaux, "Interference-free SDMA for FBMC-OQAM," EURASIP Journal on Advances in Signal Processing, vol. 2013:46, no. 1, pp. 1-13, 2013.

[98] M. Newinger, L. G. Baltar, A. Swindlehurst, and J. A. Nossek, "MISO broadcasting FBMC system for highly frequency selective channels," in 18th International ITG Workshop on Smart Antennas (WSA), Erlangen, Germany, March 2014.

[99] Y. Cheng, M. Haardt, L. G. Baltar, and J. A. Nossek, "Downlink per-user multi-streaming for FBMC/OQAM based multi-user MIMO with highly frequency selective channels," in 19th International ITG Workshop on Smart Antennas, Ilmenau, Germany, March 2015.

[100] M. Caus, A. Pérez-Neira, and M. Moretti, "SDMA for FBMC with block diagonalization," in Proc. IEEE 14th International Workshop on Signal Processing Advances in Wireless Communications (SPAWC2013), Darmstadt, Germany, June 2013.

[101] R. Fischer, C. Windpassinger, A. Lampe, and J. Huber, "Space-time transmission using Tomlinson-Harashima precoding," in 4th Int. ITG Conf., Erlangen, Germany, Jan. 2002.

[102] _ " "MIMO precoding for decentralized receivers," in Proc. International Symposium on Information Theory (ISIT-2002), Lausanne, Switzerland, June 2002.

[103] M. Caus and A. Pérez-Neira, "SDMA for filterbank with TomlinsonHarashima precoding," in Proc. International Conference on Communications (ICC), Budapest, Hungary, June 2013.

[104] Y. Cheng, V. Ramireddy, and M. Haardt, "Non-linear precoding for the downlink of FBMC/OQAM based multi-user MIMO systems," in 19th International ITG Workshop on Smart Antennas, Ilmenau, Germany, March 2015.

[105] Y. Cheng, P. Li, and M. Haardt, "FBMC/OQAM for the asynchronous multi-user MIMO uplink," in Proceedings of 18th International ITG Workshop on Smart Antennas, Erlangen, Germany, March 2014.

[106] F. Rottenberg, Y. Medjahdi, E. Kofidis, and J. Louveaux, "Preamblebased channel estimation in asynchronous FBMC-OQAM distributed
MIMO systems," in 12th Int'l Symp. Wireless Communication Systems (ISWCS-2015), Brussels, Belgium, Aug. 2015.

[107] Y. Cheng, S. Li, J. Zhang, F. Roemer, B. Song, M. Haardt, Y. Zhou, and M. Dong, "An Efficient and Flexible Transmission Strategy for the Multi-carrier Multi-user MIMO Downlink," IEEE Trans. Veh. Technol., vol. 63, no. 2, pp. 628-642, Feb. 2014

[108] E. Kofidis, D. Katselis, A. Rontogiannis, and S. Theodoridis, "Preamble-based channel estimation in OFDM/OQAM systems: A review," Signal Process., vol. 93, no. 7, pp. 2038-2054, July 2013.

[109] E. Kofidis, "Channel estimation in filter bank-based multicarrier systems: Challenges and solutions," in 6th IEEE Int'l Symp. Commun., Control, and Signal Process. (ISCCSP-2014), Athens, Greece, May 2014.

[110] X. Mestre and E. Kofidis, "Pilot-based channel estimation for FBMC/OQAM systems under strong frequency selectivity," in Proc. ICASSP, Shanghai, China, March 2016.

[111] M. G. Bellanger, "FBMC physical layer: A primer," June 2010. [Online]. Available: http://www.ict-phydyas.org/teamspace/internalfolder/FBMC-Primer_06-2010.pdf

[112] L. G. Baltar, T. Laas, M. Newinger, A. Mezghani, and J. A. Nossek, "Enhancing spectral efficiency in advanced multicarrier techniques: A challenge," in 22nd European Signal Processing Conference (EUSIPCO-2014), Lisbon, Portugal, Sept. 2014.

[113] M. G. Bellanger, "Efficiency of filter bank multicarrier techniques in burst radio transmission," in IEEE Global Telecommunications Conference (GLOBECOM-2010), Miami, FL, Dec. 2010

[114] Q. Huang, M. Ghogho, and S. Freear, "Pilot design for MIMO OFDM systems with virtual carriers," IEEE Trans. Signal Process., vol. 57, no. 5, pp. 2024-2029, May 2009.

[115] S. Coleri, M. Ergen, A. Puri, and A. Bahai, "Channel estimation techniques based on pilot arrangement in OFDM systems," IEEE Trans. Broadcast., vol. 48, no. 3, pp. 223-229, Sept. 2002.

[116] J.-P. Javaudin, D. Lacroix, and A. Rouxel, "Pilot-aided channel estimation for OFDM/OQAM," in Proc. VTC (Spring), vol. 3, Jeju, Korea, April 2003.

[117] C. Lélé, J.-P. Javaudin, R. Legouable, A. Skrzypczak, and P. Siohan, "Channel estimation methods for preamble-based OFDM/OQAM modulations," in European Wireless, Paris, France, April 2007.

[118] C. M. Lélé, "OFDM/OQAM: Méthodes d' estimation de canal, et combinaison avec l'accès multiple CDMA ou les systèmes multiantennes," Ph.D. dissertation, Conservatoire National des Arts et Métiers (CNAM), 2008.

[119] J. Du, "Pulse shape adaptation and channel estimation in generalized frequency division multiplexing systems," Licentiate thesis, KTH, Stockholm, Sweden, Dec. 2008.

[120] E. Kofidis and D. Katselis, "Improved interference approximation method for preamble-based channel estimation in FBMC/OQAM," in 19th European Signal Processing Conf. (EUSIPCO-2011), Barcelona, Spain, 29 Aug.-2 Sept. 2011.

[121] _ "Preamble-based channel estimation in MIMO-OFDM/OQAM systems," in IEEE Int'l Conf. Signal and Image Processing Applications (ICSIPA-2011), Kuala Lumpur, Malaysia, Nov. 2011.

[122] G. Stüber, J. Barry, S. McLaughlin, Y. Li, M.-A. Ingram, and T. Pratt, "Broadband MIMO-OFDM wireless communications," Proc. IEEE, vol. 92, no. 2, pp. 271-294, Feb. 2004.

[123] M. Nájar et al., "MIMO channel matrix estimation and tracking," 2009, PHYDYAS D4.1 deliverable. [Online]. Available: http://www.ictphydyas.org/delivrables/PHYDYAS-D4.1.pdf/view

[124] H. Minn and N. Al-Dhahir, "Optimal training signals for MIMO OFDM channel estimation," IEEE Trans. Wireless Commun., vol. 5, no. 5, pp. 1158-1168, May 2006

[125] S. Taheri, M. Ghoraishi, and P. Xiao, "Overhead reduced preamblebased channel estimation for MIMO-FBMC systems," in Int'l Wireless Communications and Mobile Computing Conf. (IWCMC-2015), Dubrovnik, Croatia, Aug. 2015.

[126] J. Louveaux, A. Bourdoux, and F. Horlin, "Low feedback downlink MIMO channel estimation for distributed FBMC systems using SNR measurements," in 10th IEEE Int'l Symp. Wireless Commun. Systems (ISWCS-2013), Ilmenau, Germany, Aug. 2013.

[127] H. Bölcskei, P. Duhamel and R. Hleiss, "A subspace-based approach to blind channel identification in pulse shaping OFDM/OQAM systems," IEEE Trans. Signal Process., vol. 49, no. 7, pp. 1594-1598, July 2001.

[128] W. Hu and B. Champagne, "Semiblind channel estimation for OFDM/OQAM systems," IEEE Signal Process. Lett., vol. 22, no. 4, pp. 400-403, April 2015. 
[129] J. Du, P. Xiao, J. Wu, and Q. Chen, "Design of isotropic orthogonal transform algorithm-based multicarrier systems with blind channel estimation," IET Communications, vol. 6, no. 16, pp. 2695-2704, Nov. 2012.

[130] C. S. Lee and K. Y. Yoo, "Polyphase filter-based OFDM transmission system," in 60th IEEE Vehicular Technology Conf. (VTC-2004 Fall), Los Angeles, CA, Sept. 2004.

[131] E. Kofidis, "Preamble-based channel estimation in OFDM/OQAM systems: A time-domain approach," arXiv:1306.2581v2 [cs.IT], Jan. 2014.

[132] — "Preamble-based estimation of highly frequency selective channels in MIMO-FBMC/OQAM systems," in 21st European Wireless Conf. (EW-2015), Budapest, Hungary, May 2015.

[133] — - "Short preamble-based estimation of highly frequency selective channels in FBMC/OQAM," in 39th IEEE Int'l Conf. Acoustics, Speech and Signal Process. (ICASSP-2014), Florence, Italy, May 2014.

[134] I. Barhumi, G. Leus, and M. Moonen, "Optimal training designs for MIMO OFDM systems in mobile wireless channels," IEEE Trans. Signal Process., vol. 51, no. 6, pp. 1615-1624, June 2003.

[135] D. T. Phan-Huy, P. Siohan, and M. Hélard, "Make-it-realand-anticirculating orthogonal space-time coding for MIMO OFDM/OQAM," in Proc. SPAWC, Stockholm, Sweden, June 2015.

[136] F. Rottenberg, F. Horlin, E. Kofidis, and J. Louveaux, "Generalized optimal pilot allocation for channel estimation in multicarrier systems," in Proc. SPAWC, Edinburgh, UK, July 2016.

[137] E. Kofidis, "On optimal multi-symbol preambles for highly frequency selective FBMC/OQAM channel estimation," in 12th IEEE Int'l Symp. Wireless Communication Systems (ISWCS-2015), Brussels, Belgium, Aug. 2015.

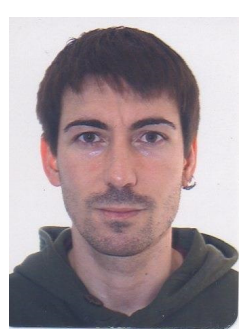

Màrius Caus received the M.Sc. and Ph.D. degrees in Telecommunications engineering from the Universitat Politècnica de Catalunya (UPC), Barcelona, Spain, in July 2008 and December 2013, respectively. In September 2009 he was granted by the Spanish Government to carry out his PhD studies in the Signal Theory and Communications Department at UPC. He has participated in several EC-funded projects (PHYDYAS, EMPhAtiC, NEWCOM\# and FANTASTIC-5G). He is currently a Postdoctoral Researcher in Centre Tecnològic de Telecomunicacions de Catalunya (CTTC), Castelldefels, Spain. His main research interests include filter bank-based multicarrier systems, signal processing for communications and satellite communications.

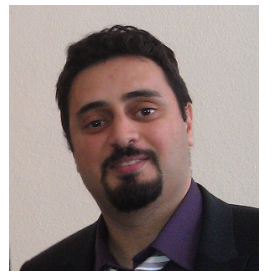

Rostom Zakaria received the engineering degree in electronic from the University of Science and Technology Houari Boumediene, Algiers, Algeria, in 2007. Then he received his M.Sc. degree from University of Paris-Est de Marne-La-Vallée, France, in 2008. From 2008 to 2012, he had been working toward the $\mathrm{PhD}$ degree in the CEDRIC/LAETITIA research laboratory at Conservatoire National des Arts et Métiers (CNAM), Paris. Since 2012, he is temporary assistant professor and researcher at CNAM Paris. His research interests are interference cancellation techniques in the filter bank-based multicarrier (FBMC) modulations, spatial time and frequency coding in FBMC, and device-to-device (D2D) communications.

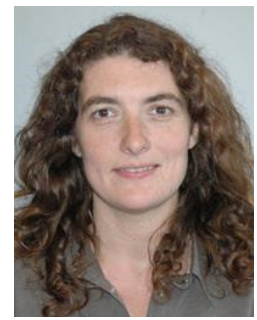

Ana I. Pérez-Neira (SM'02) is full professor at UPC (Technical University of Catalonia) in the Signal Theory and Communication department. Her research topic is signal processing for communications and currently she is working in multi-antenna and multicarrier signal processing, both, for satellite communications and wireless systems. She has been in the board of directors of ETSETB (Telecom Barcelona) from 2000-03 and Vicerector for Research at UPC (2010-13). She created UPC Doctoral School (2011). Currently, she is Scientific Coordinator at CTTC (Centre Tecnològic de Telecomunicacions de Catalunya). Since 2008 she is member of EURASIP BoD (European Signal Processing Association) and since 2010 of IEEE SPTM (Signal Processing Theory and Methods). She is the coordinator of the European project SANSA and of the Network of Excellence on satellite communications, financed by the European Space Agency: SatnexIV. She has been the leader of 20 projects and has participated in over 50 (10 for European Space Agency). She is author of 50 journal papers (20 related with Satcom) and more than 200 conference papers (20 invited). She is co-author of 4 books and 5 patents (one on satcom). She has been guest editor in 5 special issues and currently she is editor of IEEE Transactions on Signal Processing and of Eurasip Signal Processing and Advances in Signal Processing. She has been the general chairman of IWCLD'09, EUSIPCO'11, EW'14 and IWSCS'14. She has participated in the organization of ESA conference 1996, SAM'04 and she is the general chair of next ASMS'16. She is the chair of IEEE ICASSP'20.

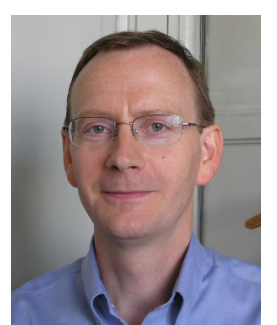

Didier Le Ruyet received his Eng. Degree and his $\mathrm{Ph}$. D. degree from Conservatoire National des Arts et Métiers (CNAM) in 1994 and 2001 respectively. In 2009, he received the "Habilitation à diriger des recherches" from Paris XIII University. From 1988 to 1996 he was a Senior Member of the Technical Staff at SAGEM Defence and Telecommunication, France. He joined Signal and Systems Laboratory, CNAM, Paris as a research assistant in 1996. From 2002 to 2010 , he was an assistant professor with the Electronic and Communication Laboratory, CNAM Paris. Since 2010 he is full professor at CNAM in the CEDRIC research laboratory. He has published more than 100 papers in refereed journals and conference proceedings and six books/book chapters in the area of communication. He has been involved in several National and European projects dealing with multicarrier transmission techniques and multi-antenna transmission. He served as Technical Program Committee member in major IEEE conferences (ICC, GLOBECOM, VTC, ISWCS, WCNC). He was General chair for ISWCS 2012 and co-General chair for ISWCS 2014. He served as a Co-editor of a special issue of Eurasip Journal on wireless communications and networking on recent advances in multiuser MIMO systems. His main research interests lie in the areas of digital communications and signal processing including channel coding, detection and estimation algorithms, filter bank based multi-carrier communication and multi-antenna transmission. He is senior member of IEEE. 


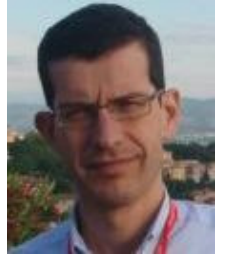

Eleftherios Kofidis (IEEE S'91, M'97) received the Diploma and Ph.D. degrees in 1990 and 1996, respectively, both from the Department of Computer Engineering and Informatics, University of Patras, Patras, Greece. From 1996 to 1998 he served in the Hellenic Army. In the period 1998 to 2000, he was a postdoctoral fellow at the Institut National des Télécommunications (INT), Evry, France (now Télécom SudParis). From 2001 to 2004, he was a research associate at the University of Athens, and adjunct professor at the Universities of Peloponnese and Piraeus, Greece. In 2004, he joined the Dept. of Statistics and Insurance Science, University of Piraeus, Greece, where he is now Assistant Professor. He is also affiliated with the Computer Technology Institute \& Press "Diophantus" (CTI), Greece. His research interests are in signal processing for communications (with emphasis on MIMO and multicarrier systems) and medical imaging. Dr. Kofidis has served as technical program co-chair in two international conferences (CIP-2008 and DSP-2009) and as a technical program committee member and reviewer in a number of conferences and journals. He has served as Associate Editor in the EURASIP Journal on Advances in Signal Processing (JASP) and the IET Signal Processing journal. $\mathrm{He}$ (co-)organized three special sessions on filter bank-based multicarrier systems (ISWCS-2012, EW-2014, SPAWC-2015) and was lead guest editor for a JASP special issue on this subject. He is currently serving as Associate Editor in the IEEE Transactions on Signal Processing journal.

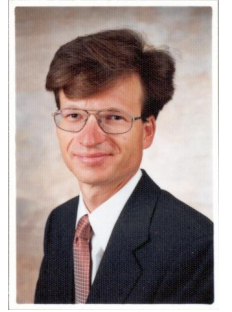

Martin Haardt (S'90, M'98, SM'99) has been a Full Professor in the Department of Electrical Engineering and Information Technology and Head of the Communications Research Laboratory at Ilmenau University of Technology, Germany, since 2001. Since 2012, he has also served as an Honorary Visiting Professor in the Department of Electronics at the University of York, UK. After studying electrical engineering at the Ruhr-University Bochum, Germany, and at Purdue University, USA, he received his Diplom-Ingenieur (M.S.) degree from the Ruhr-University Bochum in 1991 and his Doktor-Ingenieur (Ph.D.) degree from Munich University of Technology in 1996. In 1997 he joint Siemens Mobile Networks in Munich, Germany, where he was responsible for strategic research for third generation mobile radio systems. From 1998 to 2001 he was the Director for International Projects and University Cooperations in the mobile infrastructure business of Siemens in Munich, where his work focused on mobile communications beyond the third generation. During his time at Siemens, he also taught in the international Master of Science in Communications Engineering program at Munich University of Technology.

Martin Haardt has received the 2009 Best Paper Award from the IEEE Signal Processing Society, the Vodafone (formerly Mannesmann Mobilfunk) Innovations-Award for outstanding research in mobile communications, the ITG best paper award from the Association of Electrical Engineering, Electronics, and Information Technology (VDE), and the Rohde \& Schwarz Outstanding Dissertation Award. In the fall of 2006 and the fall of 2007 he was a visiting professor at the University of Nice in Sophia-Antipolis, France, and at the University of York, UK, respectively. His research interests include wireless communications, array signal processing, high-resolution parameter estimation, as well as numerical linear and multi-linear algebra. Prof. Haardt has served as an Associate Editor for the IEEE Transactions on Signal Processing (2002-2006 and 2011-2015), the IEEE Signal Processing Letters (2006-2010), the Research Letters in Signal Processing (2007-2009), the Hindawi Journal of Electrical and Computer Engineering (since 2009), the EURASIP Signal Processing Journal (2011-2014), and as a guest editor for the EURASIP Journal on Wireless Communications and Networking. Since 2011 he has been an elected member of the Sensor Array and Multichannel (SAM) technical committee of the IEEE Signal Processing Society, where he currently serves as the Vice Chair (since 2015). Moreover, he has served as the technical co-chair of PIMRC 2005 in Berlin, Germany, ISWCS 2010 in York, UK, as well as the European Wireless 2014 in Barcelona, and as the general co-chair of ISWCS 2013 in Ilmenau, Germany, CAMSAP 2013 in Saint Martin, French Antilles, WSA 2015 in Ilmenau, SAM 2016 in Rio de Janeiro, Brazil, and CAMSAP 2017 in Curacao, Dutch Antilles.

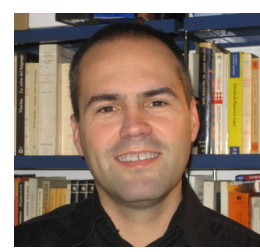

Xavier Mestre (S'96, M'03, SM'09) received the $\mathrm{MS}$ and $\mathrm{PhD}$ in Electrical Engineering in 1997 and 2002 respectively and the Licenciate Degree in Mathematics in 2011 from the same University. From January 1998 to December 2002, he was with UPC's Communications Signal Processing Group, where he worked as a Research Assistant. In January 2003 he joined the Telecommunications Technological Center of Catalonia (CTTC), where he currently holds a position as a Senior Research Associate in the area of Radio Communications. During this time, he has actively participated in multiple European projects and several contracts with the local industry. He has been the coordinator of the European FP7 EMPhAtiC project (2012-15). Currently, he is head of the Advanced Signal and Information Processing Department. He has served as Technical Chair of EUSIPCO 2011, General Co-Chair of the European Wireless 2014 and general chair of several seasonal schools. He will serve as general co-chair of ICASSP 2020 in Barcelona. He has been associate editor of IEEE Transactions on Signal Processing, 2007-2011, 2015-present and associate co-editor of the special issue on Cooperative Communications in Wireless Networks at the EURASIP Journal on Wireless Communications and Networking. He is an elected member of the IEEE Sensor Array and Multichannel Signal Processing Technical Committee and member of the EURASIP Special Area Team on Theoretical and Methodological Trends in Signal Processing.

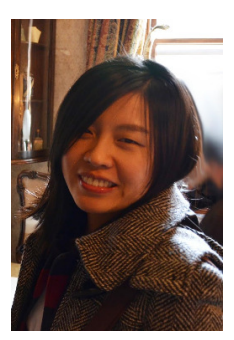

Yao Cheng (S'12) received her Bachelor's degree in communications engineering from Shandong University, China, and the Master's degree in communications engineering at University of Ulm, Germany, in 2008 and 2010, respectively. Since October 2010, she has been a research assistant in the Communications Research Laboratory at Ilmenau University of Technology. Her research interests include multiuser MIMO precoding and scheduling, multidimensional signal processing, as well as filter-bank-based multi-carrier. 